ANALYSIS \& PDE

Volume $5 \quad$ No. $3 \quad 2012$

JONATHAN LUK

A VECTOR FIELD METHOD APPROACH TO IMPROVED DECAY FOR SOLUTIONS TO THE WAVE EQUATION ON A SLOWLY ROTATING KERR BLACK HOLE 


\title{
A VECTOR FIELD METHOD APPROACH TO IMPROVED DECAY FOR SOLUTIONS TO THE WAVE EQUATION ON A SLOWLY ROTATING KERR BLACK HOLE
}

\author{
JONATHAN LUK
}

\begin{abstract}
We prove that sufficiently regular solutions to the wave equation $\square_{g_{K}} \Phi=0$ on the exterior of a sufficiently slowly rotating Kerr black hole obey the estimates $|\Phi| \leq C\left(t^{*}\right)^{-3 / 2+\eta}$ on a compact region of $r$. This is proved with the help of a new vector field commutator that is analogous to the scaling vector field on Minkowski and Schwarzschild spacetime. This result improves the known robust decay rates that are proved using the vector field method in the region of finite $r$ and along the event horizon.
\end{abstract}

1. Introduction 553

2. Geometry of Kerr spacetime $\quad 559$

3. Notation $\quad 562$

4. Vector field commutators $\quad 563$

5. The basic identities for currents 566

6. Statement of the main theorem $\quad 568$

7. Vector field multiplier $N_{e}$ and mild growth of nondegenerate energy 568

8. Integrated decay estimates and boundedness of nondegenerate energy 571

9. Vector field multiplier $Z$ and decay of nondegenerate energy 586

10. Estimates for solutions to $\square_{g_{K}} \Phi=0 \quad 601$

11. Estimates for $\hat{Y} \Phi$ and elliptic estimates $\quad 604$

12. Estimates for $\widetilde{\Omega} \Phi \quad 612$

13. Estimates for $S \Phi \quad 614$

14. Improved decay for the linear homogeneous wave equation 621

$\begin{array}{ll}\text { 15. Discussion } & 623\end{array}$

Acknowledgments $\quad 623$

$\begin{array}{ll}\text { References } & 623\end{array}$

\section{Introduction}

A major open problem in general relativity is that of the nonlinear stability of Kerr spacetimes. These spacetimes are stationary axisymmetric asymptotically flat black hole solutions to the vacuum Einstein equations

$$
R_{\mu \nu}=0
$$

MSC2010: 83C99, 35R01.

Keywords: Kerr spacetime, wave equation, decay estimates. 
in 3+ 1 dimensions. They are parametrized by $(M, a)$ representing respectively the mass and the specific angular momentum of a black hole; see Section 2. It is conjectured that Kerr spacetimes are asymptotically stable. In the framework of the initial value problem, the stability of Kerr would mean that for any solution to the vacuum Einstein equations with initial data close to the initial data of a Kerr spacetime, its maximal Cauchy development has an exterior region that approaches a nearby, but possibly different, Kerr spacetime.

To study the stability of Kerr spacetimes, it is important to first understand the corresponding linear problem. One way to approach this is to study the linear scalar wave equation $\square_{g_{K}} \Phi=0$, where $g_{K}$ is the metric on a fixed Kerr background and $\square_{g_{K}}$ is the Laplace-Beltrami operator. This can be compared with the proofs of the nonlinear stability of the Minkowski spacetime in which a robust understanding of the quantitative decay properties of solutions to the linear wave equation plays a fundamental role [Christodoulou and Klainerman 1993; Lindblad and Rodnianski 2005].

The Kerr family of spacetimes contains a one-parameter subfamily known as the Schwarzschild spacetimes for which $a=0$. It is natural when studying the wave equation on Kerr spacetimes to begin by focusing on the wave equation on Schwarzschild spacetimes. Pointwise boundedness and decay of the solutions to the wave equation on Schwarzschild spacetimes has been proved in [Wald 1979; Kay and Wald 1987; Machedon and Stalker 2002; Blue and Sterbenz 2006; Dafermos and Rodnianski 2009; Kronthaler 2007; Blue and Soffer 2006; Donninger et al. 2011; Tataru 2009]. In particular, Dafermos and Rodnianski used the vector field method to show that on the exterior region of the Schwarzschild spacetimes, including along the event horizon, solutions to the linear wave equation satisfy $|\Phi| \leq C\left(t^{*}\right)^{-1}$, where $t^{*}$ is a regular coordinate (up to the event horizon) that approaches infinity towards null infinity. In an earlier work [Luk 2010], we improved this decay rate. More precisely, we showed that sufficiently regular solutions to the wave equation $\square_{g} \Phi=0$ on the Schwarzschild black hole obey the estimates $|\Phi| \leq C_{\eta}\left(t^{*}\right)^{-3 / 2+\eta}$ for any $\eta>0$ on a compact region of $r$, including along the event horizon and inside the black hole.

This paper generalizes the result above to Kerr spacetimes where $a \ll M$. For Kerr spacetimes satisfying this condition, Dafermos and Rodnianski [2011], and subsequently Andersson and Blue [2009], have proved a decay rate in the exterior region of the Kerr spacetime, including along the event horizon, of $|\Phi| \leq C\left(t^{*}\right)^{-1+\eta}$, where $t^{*}$ is a regular coordinate to be defined later, and with $t^{*}$ we will define a foliation of the exterior region of Kerr spacetime by the spacelike hypersurfaces $\Sigma_{t^{*}}$. Extending the methods in [Luk 2010], we are able to improve this decay rate using the vector field method.

Theorem 1. Suppose $\square_{g_{K}} \Phi=0$. Then for all $\eta>0$ and all $M>0$ there exists $a_{0}$ such that the following estimates hold on Kerr spacetimes with $(M, a)$ for which $a \leq a_{0}$.

(1) Improved decay of nondegenerate energy:

$$
\sum_{j=0}^{M} \int_{\Sigma_{t^{*}} \cap\{r \leq R\}}\left(D^{j} \Phi\right)^{2} \leq C_{R} E_{M}\left(t^{*}\right)^{-3+\eta}
$$


(2) Improved pointwise decay:

$$
\sum_{j=0}^{M}\left|D^{j} \Phi\right| \leq C_{R} E_{M}^{\prime}\left(t^{*}\right)^{-3 / 2+\eta} \quad \text { for } r \leq R
$$

Here, $D$ denotes derivatives in a regular coordinate system (See Section 2). $E_{M}$ and $E_{M}^{\prime}$ depend only on $M$ and some weighted Sobolev norm of the initial data.

A more precise version of this theorem will be given in Section 6. Our proof relies on an analogue of the scaling vector field on Minkowski spacetime. Recall that in Minkowski spacetime the vector field $S=t \partial_{t}+r \partial_{r}$ is conformally Killing and satisfies $\left[\square_{m}, S\right]=2 \square_{m}$. Hence any estimates that hold for $\Phi$ a solution to $\square_{m} \Phi=0$ would also hold for $S \Phi$. However, $S$ has a weight that is increasing with $t$. Hence one can hope to prove a better estimate for $\Phi$ using the estimates for $S \Phi$. (See, for example, [Klainerman and Sideris 1996]).

In [Luk 2010], we introduced an analogue of the scaling vector field on Schwarzschild spacetimes. We defined, in the Regge-Wheeler tortoise coordinate (see Section 2), the vector field $S=t \partial_{t}+r^{*} \partial_{r^{*}}$. In [Luk 2010], we studied the commutator $\left[\square_{g_{S}}, S\right]$ and showed that all the error terms can be controlled. Thus, up to a loss of $t^{\eta}$ (for $\eta$ arbitrarily small), $S \Phi$ obeys all the estimates of $\Phi$ that were proved in [Dafermos and Rodnianski 2009]. In particular, we showed that $S \Phi$, like $\Phi$ itself, obeys a local integrated decay estimate

$$
\begin{array}{cc}
\int_{t^{\prime}}^{t} \int_{r_{1}}^{r_{2}}\left(D^{k} \Phi\right)^{2} d r d t \leq C E_{k}\left(t^{\prime}\right)^{-2} & \text { for } t^{\prime} \leq t \leq(1.1) t^{\prime} \\
\int_{t^{\prime}}^{t} \int_{r_{1}}^{r_{2}}\left(S D^{k} \Phi\right)^{2} d r d t \leq C E_{k}\left(t^{\prime}\right)^{-2+\eta} & \text { for } t^{\prime} \leq t \leq(1.1) t^{\prime}
\end{array}
$$

From this we proved an improved decay of the $L^{2}$ norm of $D^{k} \Phi$. We will explain the main idea in the case $k=0$. Firstly, the local integrated decay for $\Phi$ would already imply on a sequence of $t_{i}$ slices, with $t_{i} \leq t_{i+1} \leq(1.1)^{2} t_{i}$, that $\Phi$ obeys a better decay rate, namely $\Phi\left(t_{i}\right) \leq C t_{i}^{-3 / 2}$. We then introduced a new method to use the estimates for $S \Phi$, which can be explained heuristically as follows. Given any time $t$, we find the largest $t_{i} \leq t$ that has a better decay rate. Then we integrated from $t_{i}$ to $t$ using the vector field $S$. At this point $S$ has a weight that grows like $t$. Hence we have, at least schematically,

$$
\int_{r_{1}}^{r_{2}} \Phi(t)^{2} d r \leq C\left(\int_{r_{1}}^{r_{2}} \Phi\left(t_{i}\right)^{2} d r+t^{-1}\left|\int_{t_{i}}^{t} \int_{r_{1}}^{r_{2}} S\left(\Phi^{2}\right) d r d t\right|\right) .
$$

We then notice that the last term can be estimated by the local integrated decay estimates

$$
\left|\int_{t_{i}}^{t} \int_{r_{1}}^{r_{2}} S\left(\Phi^{2}\right) d r d t\right| \leq C\left(\int_{t_{i}}^{t} \int_{r_{1}}^{r_{2}} \Phi^{2} d r d t+\int_{t_{i}}^{t} \int_{r_{1}}^{r_{2}}(S \Phi)^{2} d r d t\right) \leq C t^{-2+\eta} .
$$

Putting these together, we would get

$$
\int_{r_{1}}^{r_{2}} \Phi(t)^{2} d r \leq C t^{-3+\eta}
$$


Using this method, we also showed the improved decay for the $L^{2}$ norm of higher derivatives. Pointwise decay estimate thus followed from standard Sobolev embedding.

In this paper we would like to carry out a similar argument. We introduce a scaling vector field (which we again call $S$ ) which is the same as in [Luk 2010] at the asymptotically flat end, but is smooth up to and across the event horizon. We will prove a local integrated decay estimate for $S \Phi$ and use the argument in [ibid.] as outlined above to prove an improved decay rate. The most difficult part of the argument is to control the error terms coming from the commutation of $\square_{g_{K}}$ and (the modified) $S$, that is, the term $\left[\square_{g_{K}}, S\right] \Phi$. To control this, we need to use estimates for derivatives of $\Phi$, which in turn are provided by the energy estimates for the homogeneous equation $\square_{g_{K}} \Phi=0$ proved in [Dafermos and Rodnianski $2011 ; 2008]$. This term schematically looks like

$$
\left[\square_{g_{K}}, S\right] \Phi=O(1) \square_{g_{K}} \Phi+O\left(r^{-2+\delta}\right)\left(D^{2} \Phi+D \Phi+r D \not \mathbf{D} \Phi\right)
$$

where $\not$ is an angular derivative on the 2 -sphere. The term $O(1) \square_{g_{K}} \Phi$ vanishes since we are considering $\square_{g_{K}} \Phi=0$. The other terms have the two desirable features. First, although $S$ has a weight in $t^{*}$, the commutator is independent of $t^{*}$, which is a result of $\partial_{t^{*}}$ being a Killing vector field. Second, these terms decay as $r \rightarrow \infty$, which is a result of the asymptotic flatness of Kerr spacetimes. The last term would appear to have less decay in $r$, which is also the case in Schwarzschild spacetimes. In that case, we controlled this term in [Luk 2010] by commuting the equation with $\Omega$, the generators of the spherical symmetry of Schwarzschild spacetimes. The quantity $\Omega \Phi$ would then give us control over an extra power of $r$. One difficulty that arises in the case of Kerr spacetimes is that they are not spherically symmetric. Nevertheless, following [Dafermos and Rodnianski 2008], we can construct an analog of $\Omega$, call it $\widetilde{\Omega}$, that is an asymptotic symmetry, that is, the commutator $\left[\square_{g_{K}}, \widetilde{\Omega}\right]$ would decay in $r$. The nondegenerate energy of $\widetilde{\Omega} \Phi$ would then control the last term in the above expression. Moreover, it is sufficient to define $\widetilde{\Omega}$ only when $r$ is very large since otherwise the factor in $r$ can be absorbed by constants. However, in a finite region of $r$, the commutator $\left[\square_{g_{K}}, S\right]$ would in general be large.

To understand which quantities of $S \Phi$ have to be controlled, we rederive the energy estimates in [Dafermos and Rodnianski 2008] in the slightly more general case of the inhomogeneous equation $\square_{g_{K}} \Phi=G$. This would also immediately imply that for the linear inhomogeneous equation $\square_{g_{K}} \Phi=G$ with sufficiently regular and sufficiently decaying (both in space and time) $G$, the solution $\Phi$, assuming that the initial data is sufficiently regular, would decay with a rate of $\left(t^{*}\right)^{-1+\eta}$, precisely as that in [ibid.]. We will then apply this to the equations for $\widetilde{\Omega} \Phi$ and $S \Phi$. To derive these energy estimates, we will use the (non-Killing) vector field multipliers $N$ and $Z$. Here $N$ is a modification of $\partial_{t^{*}}$ so that it is timelike everywhere, including near the event horizon. The use of $N$ tackles the issue of superradiance, a difficulty that arises from the spacelike nature of $\partial_{t^{*}}$ near the event horizon. $Z$ is an analogue of the conformal vector field $u^{2} \partial_{u}+v^{2} \partial_{v}$ in Minkowski spacetime and is used to prove decay.

Since we will use vector field multipliers that have weights in $t^{*}$ and $r$, to prove the energy estimates at $t^{*}=\tau$ for the inhomogeneous equation we would have to control the term (as well as other similar or 
more easily controlled terms)

$$
\iint_{\mathscr{R}\left(\tau_{0}, \tau\right)}\left(t^{*}\right)^{2} r^{1+\delta} G^{2}
$$

where the integration over space and the $t^{*}$ interval $\left[\tau_{0}, \tau\right]$. To prove the energy estimates for $S \Phi$, we need to show that for $G$ as in (1), this is bounded by $C(\tau)^{\eta}$. We split this into two parts: $r \leq \frac{1}{2} t^{*}$ and $r \geq \frac{1}{2} t^{*}$. For $r \leq \frac{1}{2} t^{*}$, we can replace $r^{1+\delta}$ by $r^{-3+2 \delta}$ since $G$ decays in $r$. Then, we use the fact that

$$
\sum_{k=1}^{N} \iint_{\mathscr{R}\left((1.1)^{-1} \tau, \tau\right) \cap\left\{r \leq \frac{1}{2} t^{*}\right\}} r^{-1+\delta}\left(D^{k} \Phi\right)^{2} \leq C \tau^{-2+\eta} .
$$

Hence if we sum up the whole integral by integrating in $\left[\tau_{0},(1.1) \tau_{0}\right],\left[(1.1) \tau_{0},(1.1)^{2} \tau_{0}\right]$ etc., we will get a bound of

$$
\sum_{i=0}^{\lfloor\log \tau\rfloor+1}(1.1)^{i} \tau_{0} \sim_{\eta} \tau^{\eta}
$$

For $r \geq \frac{1}{2} t^{*}$, we do not have a decay estimate for the integrated in time estimate. However, we would still have an almost boundedness estimate:

$$
\sum_{k=1}^{N} \iint_{\mathscr{R}\left((1.1)^{-1} \tau, \tau\right) \cap\left\{r \geq \frac{1}{2} t^{*}\right\}} r^{-1+\delta}\left(D^{k} \Phi\right)^{2} \leq C \tau^{\eta} .
$$

Notice, moreover, that $G^{2} \sim r^{-3+\delta}\left(D^{k} \Phi\right)^{2}$ and this region we have $r^{-3+\delta} \leq\left(t^{*}\right)^{-2} r^{-1+\delta}$. Hence we again have

$$
\sum_{k=1}^{N} \iint_{\mathscr{R}\left((1.1)^{-1} \tau, \tau\right) \cap\left\{r \geq \frac{1}{2} t^{*}\right\}} G^{2} \leq C \tau^{-2+\eta},
$$

and the required estimate followed in the same manner as in the case $r \leq \frac{1}{2} t^{*}$.

With the modified $S$, which is smooth up to the event horizon (contrary to [Luk 2010]), we can prove the improved decay estimates for the $L^{2}$ norm of $\Phi$ and $D \Phi$ once these error terms are controlled. Using the commutation with the Killing vector $\partial_{t^{*}}$, we would also have control for $L^{2}$ norm of $D \partial_{t^{*}}^{k} \Phi$. Away from the event horizon, this is sufficient to control all other derivatives by elliptic estimates. However, since near the event horizon, $\partial_{t^{*}}$ is not Killing, we would not have control over other derivatives. Here, we follow [Dafermos and Rodnianski 2008; 2011] and commute the equation with a version of the red-shift vector field, $\hat{Y}$. Once we control $D \hat{Y}^{k} \partial_{t^{*}}^{j} \Phi$ we can use the wave equation to control (any derivatives of) $\Delta \Phi$, where $\phi$ is the Laplace-Beltrami operator on the sphere, which is elliptic. We can thus control derivatives of $\Phi$ in any directions. We will show, moreover, that the commutator $\left[\square_{g_{K}}, \hat{Y}\right]$ has the property that the inhomogeneous terms can be controlled once we have controlled the $L^{2}$ norm of $D \partial_{t^{*}}^{k} \Phi$. This implies that $\hat{Y} \Phi$ would decay in the same rate as $\partial_{t^{*}} \Phi$ for which we have already derived an improved decay rate. 
We now turn to some history of this problem. We mention some results on Kerr spacetimes with $a>0$ here and refer the readers to [Dafermos and Rodnianski 2008; Luk 2010] for references on the corresponding problem on Schwarzschild spacetimes. There has been a large literature on the mode stability and nonquantitative decay of azimuthal modes. See for example [Press and Teukolsky 1973; Hartle and Wilkins 1974; Whiting 1989; Finster et al. 2008; 2006] and references in [Dafermos and Rodnianski 2008]. The first global result for the Cauchy problem was obtained by Dafermos and Rodnianski [2011], who proved that for a class of small, axisymmetric, stationary perturbations of Schwarzschild spacetime, which include Kerr spacetimes that rotate sufficiently slowly, solutions to the wave equation are uniformly bounded. Similar results were obtained later using an integrated decay estimate on slowly rotating Kerr spacetimes by Tataru and Tohaneanu [2011]. Using the integrated decay estimate, Tohaneanu [2012] also proved Strichartz estimates.

Decay for general solutions to the wave equation on sufficiently slowly rotating Kerr spacetimes was first proved by Dafermos and Rodnianski [2008] with a quantitative rate of $|\Phi| \leq C\left(t^{*}\right)^{-1+C a}$. A similar result was later obtained by Andersson and Blue [2009] using a physical space construction to obtain an integrated decay estimate. In all of [Tataru and Tohaneanu 2011; Dafermos and Rodnianski 2008; Andersson and Blue 2009], the integrated decay estimate is proved and plays an important role. All proofs of such estimates rely heavily on the separability of the wave equation, or equivalently, the existence of a Killing tensor on Kerr spacetime. In a recent work, Dafermos and Rodnianski [2010] show that assuming the integrated decay estimate (nondegenerate up to the event horizon if it exists) and boundedness for the wave equation on an asymptotically flat spacetime, the decay rate $|\Phi| \leq C\left(t^{*}\right)^{-1}$ holds. This in particular improves the rates in [Dafermos and Rodnianski 2008; Andersson and Blue 2009]. In a similar framework, but assuming in addition exact stationarity, Tataru [2009] proved a local decay rate of $\left(t^{*}\right)^{-3}$ using Fourier-analytic methods. This applies in particular to sufficiently slowly rotating Kerr spacetimes. Dafermos and Rodnianski have recently announced a proof for the decay of solutions to the wave equation on the full range of subextremal Kerr spacetimes $a<M$.

In view of the nonlinear problem, it is important to understand decay in a robust manner. In particular, past experience shows that refined decay estimates might not be stable in nonlinear problems. The vector field method is known to be robust and culminated in the proof of the stability of the Minkowski spacetime [Christodoulou and Klainerman 1993; Lindblad and Rodnianski 2005]. We prove our decay result using the vector field method with the expectation that the method will be useful for studying nonlinear problems. As a model problem, we will study the semilinear equation with a null condition on a fixed slowly rotating Kerr background. In a forthcoming paper that we will show the global existence of solutions with small initial data for this class of equations. We will also study the asymptotic behavior of these solutions. The null condition, which is a special structure of the nonlinearity, has served as an important model for the proofs of the nonlinear stability of Minkowski spacetime and we hope that it will find relevance to the problem of the nonlinear stability of Kerr spacetime.

We end the introduction with an overview of the paper. In Section 2, we will introduce the Kerr geometry, including a few different coordinate systems that we will find useful in the rest of the paper. In Section 4, we introduce the (non-Killing) vector field commutators that will be used. These include 
the scaling vector field $S$, which is the main tool for obtaining improved decay rates. In Section 5, we introduce the formalism for vector field multipliers. We then have all the notation necessary to state the precise form of our main theorem in Section 6. In Sections 7, 8 and 9, we prove the main energy estimates using the vector field multipliers $N, X$ and $Z$. We write down the energy estimates in the most general form, allowing for the possibility of controlling the inhomogeneous terms in different energy norms. Such generality is unnecessary for the result in this paper, but will be useful in studying the null condition. Starting from Section 10, we return to the homogeneous equation. In Section 10, we write down the energy estimates proved in [Dafermos and Rodnianski 2008]. We then derive the energy estimates after commuting with $\hat{Y}, \widetilde{\Omega}$ and $S$ in Sections 11,12 and 13 respectively. Finally, using the estimates for $S \Phi$, we prove the main theorem in Section 14.

\section{Geometry of Kerr spacetime}

2.1. Kerr coordinates. The Kerr metric in the Boyer-Lindquist coordinates takes the form

$$
\begin{aligned}
g_{K}=-\left(1-\frac{2 M}{r\left(1+\frac{a^{2} \cos ^{2} \theta}{r^{2}}\right)}\right) d t^{2}+\frac{1+\frac{a^{2} \cos ^{2} \theta}{r^{2}}}{1-\frac{2 M}{r}+\frac{a^{2}}{r^{2}}} d r^{2}+r^{2}\left(1+\frac{a^{2} \cos ^{2} \theta}{r^{2}}\right) d \theta^{2} \\
+r^{2}\left(1+\frac{a^{2}}{r^{2}}+\frac{2 M}{r} \frac{a^{2} \sin ^{2} \theta}{r^{2}\left(1+\frac{a^{2} \cos ^{2} \theta}{r^{2}}\right)}\right) \sin ^{2} \theta d \phi^{2}-4 M \frac{a \sin ^{2} \theta}{r\left(1+\frac{a^{2} \cos ^{2} \theta}{r^{2}}\right)} d t d \phi .
\end{aligned}
$$

In this paper, we will consider Kerr spacetimes with $a$ small. It can then be thought of as a small perturbation of Schwarzschild spacetimes because by setting $a=0$, we recover the Schwarzschild metric:

$$
g_{S}=-\left(1-\frac{2 M}{r_{S}}\right) d t_{S}^{2}+\left(1-\frac{2 M}{r_{S}}\right)^{-1} d r_{S}^{2}+r_{S}^{2} d \theta^{2}+r_{S}^{2} \sin ^{2} \theta d \phi^{2}
$$

The Cauchy development of Kerr spacetimes can be described schematically by taking a two-dimensional slice as in Figure 1.

Notice that (2) represents the metric on the exterior region (the right side in the diagram). In the coordinate system above, this is the region $\left\{r \geq r_{+}\right\}$, where $r_{+}$is the larger root of $\Delta=r^{2}-2 M r+a^{2}$. This is the region that we will study. We foliate the exterior region of the Kerr spacetime by hypersurfaces $\Sigma_{\tau}$ as depicted in the diagram. A precise definition of the hypersurface $\Sigma_{\tau}$ will be given in Section 3.3. The coordinates in (2) are not regular at the event horizon $\mathscr{H}^{+}=\left\{r=r_{+}\right\}$. It will be helpful in the sequel to use different coordinate systems on Kerr spacetimes. From now on we will call the coordinate system on which the metric (2) is defined the Kerr $(t, r, \theta, \phi)$ coordinates. We define a new coordinate system, the $\operatorname{Kerr}\left(t, r^{*}, \theta, \phi\right)$ coordinates, by

$$
\frac{d r^{*}}{d r}=\frac{r^{2}+a^{2}}{\Delta}
$$




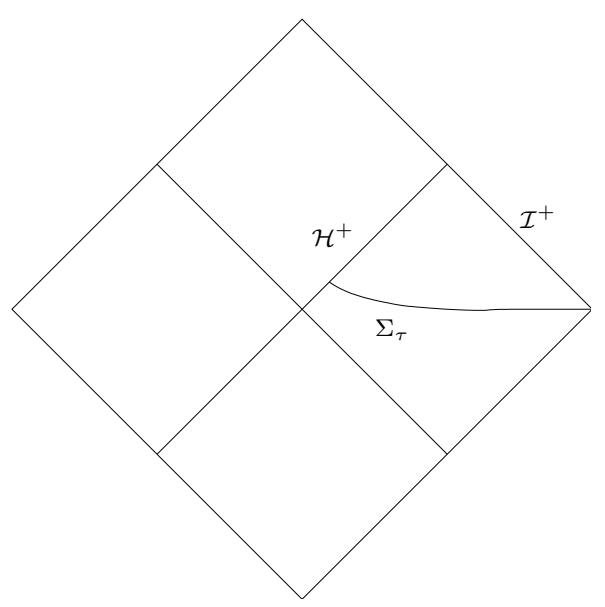

Figure 1. Kerr spacetime.

where $\Delta=r^{2}-2 M r+a^{2}$ is zero at the event horizon. In this coordinate system, the metric looks like $g_{K}=-\left(1-\frac{2 M}{r\left(1+\frac{a^{2} \cos ^{2} \theta}{r^{2}}\right)}\right) d t^{2}+\Delta\left(r^{2}+a^{2}\right)^{2}\left(r^{2}+a^{2} \cos ^{2} \theta\right) d r^{* 2}+r^{2}\left(1+\frac{a^{2} \cos ^{2} \theta}{r^{2}}\right) d \theta^{2}$
$+r^{2}\left(1+\frac{a^{2}}{r^{2}}+\frac{2 M}{r} \frac{a^{2} \sin ^{2} \theta}{r^{2}\left(1+\frac{a^{2} \cos ^{2} \theta}{r^{2}}\right)}\right) \sin ^{2} \theta d \phi^{2}-4 M \frac{a \sin ^{2} \theta}{r\left(1+\frac{a^{2} \cos ^{2} \theta}{r^{2}}\right)} d t d \phi$.

Since the definition of $r^{*}$ depends only on $r$, it is unambiguous to talk about the vector $\partial_{t}$.

2.2. Schwarzschild coordinates. In order to compare calculations on Kerr spacetimes to calculations on Schwarzschild spacetimes, it is helpful to exhibit a diffeomorphism between the two. We do so by defining an explicit map between the coordinate functions $(t, r, \theta, \phi)$ on a Kerr spacetime and the coordinate functions $\left(t_{S}, r_{S}, \theta_{S}, \phi_{S}\right)$ on a Schwarzschild spacetime with the same mass. These will be defined differently near and away from the event horizon. Take

$$
\chi(r)= \begin{cases}1 & \text { if } r \leq r_{Y}^{-}-\frac{1}{2}\left(r_{Y}^{-}-r_{+}\right) \\ 0 & \text { if } r \geq r_{Y}^{-}-\frac{1}{4}\left(r_{Y}^{-}-r_{+}\right)\end{cases}
$$

where $r_{+}$, as above, is the larger root of $\Delta=r^{2}-2 M r+a^{2}$ and $r_{Y}^{-}>r_{+}$is a constant to be determined later. With this $\chi(r)$, we can then define

$$
\begin{aligned}
& r_{S}^{2}-2 M r_{S}=r^{2}-2 M r+a^{2}, \\
& t_{S}+\chi\left(r_{S}\right) 2 M \log \left(r_{S}-2 M\right)=t+\chi(r) h(r), \quad \text { where } \frac{d h(r)}{d r}=\frac{2 M r}{r^{2}-2 M r+a^{2}}, \\
& \theta_{S}=\theta \text {, } \\
& \phi_{S}=\phi+\chi(r) P(r), \quad \text { where } \frac{d P(r)}{d r}=\frac{a}{r^{2}-2 M r+a^{2}} .
\end{aligned}
$$


Then, by identifying $\left(t_{S}, r_{S}, \theta_{S}, \phi_{S}\right)$ with the corresponding coordinate functions on Schwarzschild spacetimes, we have a diffeomorphism between Kerr spacetimes and Schwarzschild spacetimes. This coordinate system will be used and will be called the Schwarzschild $\left(t_{S}, r_{S}, \theta_{S}, \phi_{S}\right)$ coordinates on Kerr spacetimes. Once we have this diffeomorphism, we can put any system of Schwarzschild coordinates on Kerr spacetimes. These include the Schwarzschild $\left(t_{S}^{*}, r_{S}, \theta_{S}, \phi_{S}\right)$ coordinates, where $t_{S}^{*}=$ $t_{S}+\chi\left(r_{S}\right) 2 M \log \left(r_{S}-2 M\right)$ and $r_{S}, \theta_{S}, \phi_{S}$ are defined as above. We also define

$$
t^{*}=t_{S}^{*}=t_{S}+\chi\left(r_{S}\right) 2 M \log \left(r_{S}-2 M\right)
$$

and use the $\operatorname{Kerr}\left(t^{*}, r, \theta, \phi^{*}\right)$ coordinates. Notice that $\partial_{t^{*}}=\partial_{t_{S}^{*}}$.

It is common to denote on Schwarzschild spacetimes $\mu=2 M / r_{S}$. We will take the same notation on Kerr spacetimes, with the understanding that it is always defined with respect to the Schwarzschild $r_{S}$ coordinates. In particular $(1-\mu)$ approaches 0 as $r \rightarrow r_{+}$(the event horizon).

Another system of Schwarzschild coordinates can be defined by considering two coordinate charts on the standard unit 2-sphere and introducing a system of coordinates $\left(x_{S}^{A}, x_{S}^{B}\right)$ on each of them. We then define the Schwarzschild $\left(t_{S}^{*}, r_{S}, x_{S}^{A}, x_{S}^{B}\right)$ coordinates in the obvious manner. Using this coordinate system and the diffeomorphism as above, we have, for small $a$,

$$
\left|\left(g_{K}\right)_{\alpha \beta}-\left(g_{S}\right)_{\alpha \beta}\right| \leq \epsilon r^{-2} .
$$

This smallness assumption will be used throughout this paper.

2.3. Null frame near event horizon. Some extra cancellations for the estimates near the event horizon are best captured using a null frame. Hence we define a null frame $\left\{\hat{V}, \hat{Y}, E_{1}, E_{2}\right\}$ in the region $r \leq r_{Y}^{-}$, where $r_{Y}^{-}$is to be determined later. On the event horizon,

$$
V=\partial_{t^{*}}+\frac{a}{2 M r_{+}} \partial_{\phi^{*}}
$$

is the Killing null generator. A direct computation shows that it satisfies

$$
\nabla_{V} V=\kappa V
$$

where $\kappa$ is a strictly positive number on the event horizon. We want to extend $V$ to a null frame. On the event horizon, define $\hat{Y}$ first on a 2-sphere given by a fixed $t^{*}$ to be null, orthogonal to the 2-sphere and require that $g_{K}(V, \hat{Y})=-2$. Define also locally an orthonormal frame $\left\{E_{1}, E_{2}\right\}$ tangent to the fixed 2 -sphere. In the sequel, we will only need to work with a local null frame. We then extend this null frame off the fixed 2-sphere on the event horizon (with $\left.\hat{V}\right|_{\mathscr{H}}=V$ ) by requiring

$$
\nabla_{\hat{Y}} \hat{Y}=\nabla_{\hat{Y}} \hat{V}=\nabla_{\hat{Y}} E_{A}=0,
$$

where $A \in\{1,2\}$. Then extend this null frame using the isomorphisms generated by $V$. The equations above hold everywhere. If we choose $r_{Y}^{-}$close enough to $r_{+}$, we still have, by Taylor's theorem,

$$
\nabla_{\hat{V}} \hat{V}=\kappa \hat{V}+b^{Y} \hat{Y}+b^{1} E_{1}+b^{2} E_{2}
$$


where $\kappa$ is a strictly positive function in $r_{+} \leq r \leq r_{Y}^{-}$bounded away from 0 , and $\left|b^{\alpha}\right| \leq C(1-\mu)$.

In Schwarzschild spacetime, consider the frame on $\mathscr{S}^{2}$ given by $\left\{\left(r_{S}^{2} \sin \theta_{S}\right)^{-1} \partial_{\phi_{S}}, r_{S}^{-1} \partial_{\theta_{S}}\right\}$. Then we get

$$
\hat{V}=(1+\mu) \partial_{t_{S}^{*}}+(1-\mu) \partial_{r_{S}}, \quad \hat{Y}=\partial_{t_{S}^{*}}-\partial_{r_{S}}, \quad E_{1}=r_{S}^{-1} \partial_{\theta_{S}}, \quad E_{2}=\left(r_{S} \sin \theta_{S}\right)^{-1} \partial_{\phi_{S}}
$$

Since we consider Kerr spacetimes on which the metric is close to that on a Schwarzschild spacetime, the null frame can be expressed in $\left(t^{*}, r, \theta, \phi^{*}\right)$ coordinates as

$$
\begin{array}{ll}
\hat{V}=(1+\mu) \partial_{t^{*}}+(1-\mu) \partial_{r}+O_{1}(\epsilon) \partial, & E_{1}=r^{-1} \partial_{\theta}+O_{1}(\epsilon) \partial, \\
\hat{Y}=\partial_{t^{*}}-\partial_{r}+O_{1}(\epsilon) \partial, & E_{2}=(r \sin \theta)^{-1} \partial_{\phi^{*}}+O_{1}(\epsilon) \partial .
\end{array}
$$

Alternatively, if we write $E_{\alpha}$, where $\alpha=1,2,3,4$, for the null frame, we have

$$
\begin{array}{rlrl}
(1+\mu) \partial_{t^{*}}+(1-\mu) \partial_{r} & =\hat{V}+O_{1}(\epsilon) E_{\alpha}, & \partial_{\theta} & =r E_{1}+O_{1}(\epsilon) E_{\alpha} \\
\partial_{t^{*}}-\partial_{r} & =\hat{Y}+O_{1}(\epsilon) E_{\alpha}, \quad \partial_{\phi^{*}}=r \sin \theta E_{2}+O_{1}(\epsilon) E_{\alpha} .
\end{array}
$$

We also define the vector fields $\hat{V}, \hat{Y}, E_{1}, E_{2}$ outside $\left\{r \leq r_{Y}^{-}\right\}$by requiring them to be compactly supported in $\left\{r \leq r_{Y}^{+}\right\}$(for some $r_{Y}^{+}$to be determined) and invariant under the one-parameter families of isometries generated by $\partial_{t^{*}}$ and $\partial_{\phi^{*}}$. Notice that in particular there is no requirement that the vector fields form a null frame in the region $\left\{r_{Y}^{-} \leq r \leq r_{Y}^{+}\right\}$.

\section{Notation}

3.1. Constants. Throughout this paper, we will use $C$ to denote a large constant and $c$ to denote a small constant. They can be different from line to line. We will also use $A$ to denote bootstrap constants and we think of $A$ to be large, that is, $A \gg C$. We also use the notation $O_{i}(1)$ and $O_{i}(\epsilon)$ to denote terms that are bounded up to a constant by 1 and $\epsilon$, with bounds that improve by $r^{-1}$ for each derivative up to the $i$-th derivative. We will also use the notation $f \sim g$ to denote $c f \leq g \leq C f$.

There are some constants that we will choose in the proof. The following are values of $r$ in the Kerr coordinates:

$$
r_{+}<r_{Y}^{-}<r_{Y}^{+}<\frac{11}{4} M<R_{\Omega}
$$

We will fix $r_{Y}^{+}$and $r_{Y}^{-}$in Remarks and, respectively.

There are also smallness parameters that can be thought of as obeying

$$
0<\delta<\epsilon \ll \eta \ll e
$$

We use $\epsilon$ to denote the smallness of the specific angular momentum $a$ of the spacetimes that we are working on. We use $\eta \sim C \epsilon$ to denote the loss in the decay rate of the solutions to the wave equation as compared to that on Schwarzschild spacetimes. We use $e$ to construct the nondegenerate energy, and use $\delta$ and $\delta^{\prime}$ as small parameters whenever they are needed. The parameters $\delta$ and $\delta^{\prime}$ need not be fixed from line to line. 
3.2. Values of $t^{*}$. We will adopt the following as much as possible: We denote by $t^{*}$ a general value of $t^{*}$. In particular, it will be used for integration variables. We denote by $\tau$ the $t^{*}$ value for which we want an estimate and by $\tau_{0}$ the $t^{*}$ value where the initial data is posed. We will always assume $\tau_{0} \geq 1$ and the reader can think of $\tau_{0}=1$. When integrating, we will often denote the endpoints by $\tau^{\prime}$ and $\tau$. Finally, at a few places we will need to choose a particular value of $t^{*}$ in an interval. This is usually done to achieve the maximum or minimum of the energy quantities. We often denote such choices as $\tilde{\tau}$.

\subsection{Integration.}

Definition 1. Define the following sets:

- $\Sigma_{\tau}=\left\{t^{*}=\tau\right\}$.

- $\mathscr{R}\left(\tau^{\prime}, \tau\right)=\left\{\tau^{\prime} \leq t^{*} \leq \tau\right\}$.

- $\mathscr{H}\left(\tau^{\prime}, \tau\right)=\left\{r=r_{+}, \tau^{\prime} \leq t^{*} \leq \tau\right\}$.

When integrating on these sets, we will normally integrate with respect to the volume form, which we suppress. On $\Sigma_{\tau}$ the volume form is $\sqrt{\operatorname{det} g_{K} \mid \Sigma_{\tau}}$. On $\mathscr{R}\left(\tau^{\prime}, \tau\right)$, the volume form is $\sqrt{\operatorname{det} g_{K}}$. However, on the event horizon $\mathscr{H}\left(\tau^{\prime}, \tau\right)$, the surface is null and the metric is degenerate. Nevertheless, on $\mathscr{H}\left(\tau^{\prime}, \tau\right)$, the integrand will always be of the form $J_{\mu} n_{\Sigma_{\mathscr{Y}+}}^{\mu}$, where $n_{\Sigma_{\mathscr{Y}}+}^{\mu}$ is the normal to $\mathscr{H}\left(\tau^{\prime}, \tau\right)$. We will hence take the volume form corresponding to the (arbitrarily) chosen normal. Occasionally, we will also integrate over the topological 2-spheres given by fixing $t$ and $r$. We will denote the volume form by $d A=$ $\sqrt{\operatorname{det} g_{K} \mid \varsigma^{2}}$.

For some computations, however, it is more convenient to write down the volume form explicitly in coordinates. In our notation, the following two expressions denote the same integral:

$$
\int_{\Sigma_{\tau}} f=\int_{\Sigma_{\tau}} f \sqrt{\left.\operatorname{det} g_{K}\right|_{\Sigma_{\tau}}} d r d \theta d \phi
$$

When we write the integrals, we will often use $\iint$ to denote an integral over a spacetime region and use $\int$ denote an integral over a spacelike or null hypersurface.

The volume form on $\Sigma_{t^{*}}$ can be compared with that on $\mathscr{R}\left(\tau^{\prime}, \tau\right)$. In particular, we have

$$
\iint_{\mathscr{R}\left(\tau^{\prime}, \tau\right)} f \sim \int_{\tau^{\prime}}^{\tau}\left(\int_{\Sigma_{t^{*}}} f\right) d t^{*}
$$

\section{Vector field commutators}

In this section, we discuss the vector field commutators that we will use in this article. One obvious such vector field is the Killing vector field $\partial_{t^{*}}$, which satisfies

$$
\left[\square_{g_{K}}, \partial_{t^{*}}\right]=0 .
$$

In addition to $\partial_{t^{*}}$, we will use three non-Killing vector fields $S, \hat{Y}$ and $\Omega_{i}$ to control higher derivatives of the solution $\Phi$. We introduce $S$, a new vector field, to obtain the improved decay rate for the solution $\Phi$. We will follow [Dafermos and Rodnianski 2008; 2011], defining the commutator $\hat{Y}$ to estimate $\Phi$ near 
the event horizon. We will use the vector fields $\Omega_{i}$, which are analogues of the angular momentum vector fields in Schwarzschild spacetime, to control the error terms coming from the commutator $\left[\square_{g_{K}}, S\right]$.

4.1. Vector field commutators under metric perturbations. Some computations are easier in Schwarzschild spacetime than in Kerr spacetime. In the sequel, we will often consider a fixed vector field on the differentiable structure of the Schwarzschild exterior. We now show that for such vector fields, the commutators with $\square_{g_{S}}$ and $\square_{g_{K}}$ are close to each other as long as $a$ is chosen to be sufficiently small:

Proposition 2. Consider either the Schwarzschild $\left(t_{S}^{*}, r_{S}, x_{S}^{A}, x_{S}^{B}\right)$ coordinates or $\left(t_{S}, r_{S} \geq r_{Y}^{-}, x_{S}^{A}, x_{S}^{B}\right)$ coordinates. Suppose $V$ is a vector field defined on either of these coordinates. Then

$$
\left|\left[\square_{g_{K}}-\square_{g_{S}}, V\right] \Phi\right| \leq C \epsilon r^{-2}\left(\sum_{m=1}^{2} \sum_{k=1}^{2} \max _{\alpha}\left|\partial^{m} V^{\alpha}\right|\left|\partial^{k} \Phi\right|\right),
$$

where $\partial$ is the coordinate derivative for the coordinate system on which $V$ is defined.

Proof. We rewrite

$$
\square_{g_{S}}=g_{S}^{\alpha \beta} \partial_{\alpha} \partial_{\beta}+\eta_{S}^{\alpha} \partial_{\alpha} \quad \text { and } \quad \square_{g_{K}}=g_{K}^{\alpha \beta} \partial_{\alpha} \partial_{\beta}+\eta_{K}^{\alpha} \partial_{\alpha} .
$$

Using $\left|\left(g_{K}\right)_{\alpha \beta}-\left(g_{S}\right)_{\alpha \beta}\right| \leq \epsilon r^{-2}$ and $\left|\partial_{\gamma}\left(\left(g_{K}\right)_{\alpha \beta}-\left(g_{S}\right)_{\alpha \beta}\right)\right| \leq \epsilon r^{-2}$, we have $\left|\sqrt{-\operatorname{det} g_{K}}-\sqrt{-\operatorname{det} g_{S}}\right| \leq$ $\epsilon r^{-2}$ and $\left|\partial_{\alpha}\left(\sqrt{-\operatorname{det} g_{K}}-\sqrt{-\operatorname{det} g_{S}}\right)\right| \leq \epsilon r^{-2}$. Therefore,

$$
\sup _{\alpha, \beta}\left|g_{S}^{\alpha \beta}-g_{K}^{\alpha \beta}\right|+\sup _{\alpha}\left|\eta_{S}^{\alpha}-\eta_{K}^{\alpha}\right| \leq C \epsilon r^{-2} .
$$

Therefore,

$$
\begin{aligned}
\left|\left[\square_{g_{K}}-\square_{g_{S}}, V\right] \Phi\right| & \leq\left|\left(g_{K}^{\alpha \beta}-g_{S}^{\alpha \beta}\right)\left(\partial_{\alpha} \partial_{\beta} V^{\gamma}\right) \partial_{\gamma} \Phi\right|+2\left|\left(g_{K}^{\alpha \beta}-g_{S}^{\alpha \beta}\right) \partial_{\alpha} V^{\gamma} \partial_{\beta} \partial_{\gamma} \Phi\right| \\
& +\left|\left(\eta_{S}^{\alpha}-\eta_{K}^{\alpha}\right)\left(\partial_{\alpha} V^{\gamma}\right) \partial_{\gamma} \Phi\right| \\
& \leq C \epsilon r^{-2}\left(\sum_{m=1}^{2} \sum_{k=1}^{2} \sup _{\alpha}\left|\partial^{m} V^{\alpha}\right|\left|\partial^{k} \Phi\right|\right) .
\end{aligned}
$$

4.2. Commutator $S$. We construct a commuting vector field $S$ on Schwarzschild that is different from [Luk 2010] and is stable under perturbation.

Define $S=t_{S}^{*} \partial_{t_{S}^{*}}+h\left(r_{S}\right) \partial_{r_{S}}$, where

$$
h(r)= \begin{cases}r-2 M & \text { if } r \sim 2 M, \\ (r+2 M \log (r-2 M)-3 M-2 M \log M)(1-\mu) & \text { if } r \geq R,\end{cases}
$$

for some large $R$, and it is interpolated so that it is smooth and nonnegative. For $r \geq R$, since $t^{*}=t$, this agrees with the definition in [Luk 2010]. Therefore we have

$$
\left[\square_{g S}, S\right]=\left(2+\frac{r^{*} \mu}{r}\right) \square_{g_{S}}+\frac{2}{r}\left(\frac{r^{*}}{r}-1-\frac{2 r^{*} \mu}{r}\right) \partial_{r^{*}}+2\left(\left(\frac{r^{*}}{r}-1\right)-\frac{3 r^{*} \mu}{2 r}\right) \not \Delta,
$$

where $\phi$ is the Laplace-Beltrami operator on the standard sphere. In the coordinates $\left(t^{*}, r, \theta, \phi\right)$,

$$
\square_{g_{S}}=-\alpha_{1}(r) \partial_{t}^{2}+\alpha_{2}(r) \partial_{r}^{2}+\alpha_{3}(r) \partial_{r} \partial_{t}+\alpha_{4}(r) \partial_{t}+\alpha_{5}(r) \partial_{r}+\Delta .
$$


The crucial observation is that all $\alpha_{i}$ are smooth and bounded and depend only on $r$. Noting that $\alpha_{i}$ does not depend on $t$, we have

$$
\left[\square_{g_{S}}, S\right]=\beta_{1}(r) \partial_{t}^{2}+\beta_{2}(r) \partial_{r}^{2}+\beta_{3}(r) \partial_{r} \partial_{t}+\beta_{4}(r) \partial_{t}+\beta_{5}(r) \partial_{r}+\beta_{6}(r) \not \Delta .
$$

Again, it is important to note that all $\beta_{i}$ are smooth, bounded and depend only on $r$. The form of $\beta_{i}$ for $r \geq R$ is given by (6).

We consider the same vector field $S$ on Kerr. Using Proposition 2, and noting that $\partial^{m} S^{\alpha}$ is bounded for $m \geq 1$, we have for $r>R$

$$
\begin{array}{r}
\left|\left[\square_{g_{K}}, S\right] \Phi-\left(2+\frac{r^{*} \mu}{r}\right) \square_{g_{K}} \Phi-\frac{2}{r}\left(\frac{r^{*}}{r}-1-\frac{2 r^{*} \mu}{r}\right) \partial_{r^{*}} \Phi-2\left(\left(\frac{r^{*}}{r}-1\right)-\frac{3 r^{*} \mu}{2 r}\right) \phi \Phi\right| \\
\leq C \epsilon r^{-2}\left(\sum_{k=1}^{2}\left|\partial^{k} \Phi\right|\right),
\end{array}
$$

and for $r \leq R$,

$$
\left|\left[\square_{g_{K}}, S\right] \Phi\right| \leq C \sum_{k=1}^{2}\left|D^{k} \Phi\right| .
$$

4.3. Commutator $\tilde{\Omega}_{i}$. Let $\Omega_{i}$ be a basis of vector fields of rotations in Schwarzschild spacetimes. An explicit realization can be

$$
\Omega=\left\{\partial_{\phi}, \sin \phi \partial_{\theta}+\frac{\cos \phi \cos \theta}{\sin \theta} \partial_{\phi}, \cos \phi \partial_{\theta}-\frac{\sin \phi \cos \theta}{\sin \theta} \partial_{\phi}\right\} .
$$

Define $\widetilde{\Omega}_{i}=\chi(r) \Omega_{i}$ to be cutoff so that it is supported in $\left\{r>R_{\Omega}\right\}$ and equals $\Omega_{i}$ for $r>R_{\Omega}+1$ for some large $R_{\Omega}$. On Schwarzschild spacetimes, $\Omega_{i}$ is Killing and therefore $\widetilde{\Omega}_{i}$ is Killing for $r>R_{\Omega}+1$. Therefore,

$$
\left[\square_{g s}, \widetilde{\Omega}_{i}\right]=\tilde{\chi}(r)\left(\partial^{2}+\partial\right),
$$

where $\tilde{\chi}$ is some function that depends only on $r$ and is supported in $\left\{R_{\Omega}<r<R_{\Omega}+1\right\}$.

Using Proposition 2, we have

$$
\left|\left[\square_{g_{K}}, \widetilde{\Omega}_{i}\right] \Phi\right| \leq C r^{-2}\left(\left|\partial^{2} \Phi\right|+|\partial \Phi|\right) .
$$

Moreover, since $\widetilde{\Omega}_{i}$ vanishes for $r<R_{\Omega}$, we have trivially

$$
\left[\square_{g_{K}}, \widetilde{\Omega}_{i}\right] \Phi=0 \quad \text { for } r<R_{\Omega} .
$$

From now on, we write $\widetilde{\Omega}$ to denote any one of the $\Omega_{i}$, while taking the norm to be $|\widetilde{\Omega} \Phi|=\sum_{i}\left|\Omega_{i} \Phi\right|$. This commutator is useful for gaining powers of $r$ near spatial infinity. In particular we have

$$
|\not| \Phi\left|\leq C r^{-1}\right| \widetilde{\Omega} \Phi \mid \text {. }
$$

This extra power of $r$ is essential for controlling the error terms arising from the commutation of $\square_{g_{K}}$ with $S$. 
4.4. Commutator $\hat{Y}$. Let $\hat{Y}$, as in Section 2.3 , be a vector field that is null near the event horizon, is normalized with respect to another null vector $\hat{V}$ and is cut off to be compactly supported in $\left\{r \leq r_{Y}^{+}\right\}$.

Proposition 3. On Kerr spacetimes such that $\epsilon$ is small, we have

$$
\left|\left[\square_{g_{K}}, \hat{Y}\right] \Phi-\kappa \hat{Y}^{2} \Phi\right| \leq C\left(\left|D \partial_{t^{*}} \Phi\right|+\epsilon\left|D^{2} \Phi\right|+|D \Phi|\right) \quad \text { for } r \leq r_{Y}^{-},
$$

where $\kappa>c>0$ is as in (5).

Proof. The principal term for the commutator $\left[\square_{g_{K}}, \hat{Y}\right] \Phi$ is $2^{(\hat{Y})} \pi^{\mu v} D_{\mu} D_{\nu} \Phi$, where ${ }^{(\hat{Y})} \pi_{\mu \nu}$ is the deformation tensor defined by ${ }^{(\hat{Y})} \pi_{\mu \nu}=\frac{1}{2}\left(D_{\mu} \hat{Y}_{\nu}+D_{\nu} \hat{Y}_{\mu}\right)$. We look at three terms that are useful in deriving the estimates.

$$
\begin{aligned}
{ }^{(\hat{Y})} \pi_{\hat{V} \hat{V}} & =g\left(D_{\hat{V}} \hat{Y}, \hat{V}\right)=-g\left(\hat{Y}, D_{\hat{V}} \hat{V}\right)=2 \kappa, \\
\left|{ }^{(\hat{Y})} \pi_{\hat{V} E_{A}}\right| & =\left|\frac{1}{2}\left(g\left(D_{\hat{V}} \hat{Y}, E_{A}\right)+g\left(D_{E_{A}} \hat{Y}, \hat{V}\right)\right)\right| \leq C \epsilon, \\
\left|{ }^{(\hat{Y})} \pi_{E_{A} E_{B}}\right| & =\left|\frac{1}{2}\left(g\left(D_{E_{B}} \hat{Y}, E_{A}\right)+g\left(D_{E_{A}} \hat{Y}, E_{B}\right)\right)\right| \leq C \epsilon,
\end{aligned}
$$

where the smallness in the second and third line come from the assumption that we are close to Schwarzschild. Notice also that for $r \leq r_{Y}^{-}, \hat{V}$ is $C^{0}$ close to $\partial_{t^{*}}$. Therefore, in the commutator, the main term is

$$
\kappa \hat{Y}^{2} \Phi
$$

All the other second order terms either have a $\partial_{t^{*}}$ derivative or small.

\section{The basic identities for currents}

5.1. Vector field multipliers. We consider the conservation laws for $\Phi$ satisfying $\square_{g} \Phi=0$. Define the energy-momentum tensor

$$
T_{\mu \nu}=\partial_{\mu} \Phi \partial_{\nu} \Phi-\frac{1}{2} g_{\mu \nu} \partial^{\alpha} \Phi \partial_{\alpha} \Phi .
$$

We note that $T_{\mu \nu}$ is symmetric and the wave equation implies that $D^{\mu} T_{\mu \nu}=0$. Given a vector field $V^{\mu}$, we define the associated currents

$$
J_{\mu}^{V}(\Phi)=V^{\nu} T_{\mu \nu}(\Phi) \quad \text { and } \quad K^{V}(\Phi)={ }^{(V)} \pi_{\mu \nu} T^{\mu \nu}(\Phi),
$$

where ${ }^{(V)} \pi_{\mu \nu}$ is the deformation tensor defined by

$$
{ }^{(V)} \pi_{\mu \nu}=\frac{1}{2}\left(D_{\mu} V_{\nu}+D_{v} V_{\mu}\right)
$$

In particular, $K^{V}(\Phi)={ }^{(V)} \pi_{\mu \nu}=0$ if $V$ is Killing. Since the energy-momentum tensor is divergence free,

$$
D^{\mu} J_{\mu}^{V}(\Phi)=K^{V}(\Phi) .
$$

We also define the modified currents

$$
\begin{aligned}
& J_{\mu}^{V, w}(\Phi)=J_{\mu}^{V}(\Phi)+\frac{1}{8}\left(w \partial_{\mu} \Phi^{2}-\partial_{\mu} w \Phi^{2}\right), \\
& K^{V, w}(\Phi)=K^{V}(\Phi)+\frac{1}{4} w \partial^{\nu} \Phi \partial_{\nu} \Phi-\frac{1}{8} \square_{g} w \Phi^{2} .
\end{aligned}
$$


Then

$$
D^{\mu} J_{\mu}^{V, w}(\Phi)=K^{V, w}(\Phi)
$$

We integrate by parts with this in the region bounded by $\Sigma_{\tau}, \Sigma_{\tau^{\prime}}$ and $\mathscr{H}^{+}\left(\tau^{\prime}, \tau\right)$. We denote this region as $\mathscr{R}\left(\tau^{\prime}, \tau\right)$. We denote the future-directed normal to $\Sigma_{\tau}$ by $n_{\Sigma_{\tau}}^{\mu}$.

Proposition 4. We have

$$
\begin{gathered}
\int_{\Sigma_{\tau}} J_{\mu}^{V}(\Phi) n_{\Sigma_{\tau}}^{\mu}+\int_{\mathscr{H}\left(\tau^{\prime}, \tau\right)} J_{\mu}^{V}(\Phi) n_{\mathscr{H}+\left(\tau^{\prime}, \tau\right)}^{\mu}+\iint_{\mathscr{R}\left(\tau^{\prime}, \tau\right)} K^{V}(\Phi)=\int_{\Sigma_{\tau^{\prime}}} J_{\mu}^{V}(\Phi) n_{\Sigma_{\tau^{\prime}}}^{\mu}, \\
\int_{\Sigma_{\tau}} J_{\mu}^{V, w^{V}}(\Phi) n_{\Sigma_{\tau}}^{\mu}+\int_{\mathscr{H}^{+}\left(\tau^{\prime}, \tau\right)} J_{\mu}^{V, w^{V}}(\Phi) n_{\mathscr{H}^{+}\left(\tau^{\prime}, \tau\right)}^{\mu}+\iint_{\mathscr{R}\left(\tau^{\prime}, \tau\right)} K^{V, w^{V}}(\Phi)=\int_{\Sigma_{\tau^{\prime}}} J_{\mu}^{V, w^{V}}(\Phi) n_{\Sigma_{\tau^{\prime}}}^{\mu} .
\end{gathered}
$$

One can similarly define the quantities above for the inhomogeneous wave equation $\square_{g} \Phi=F$. In this case, the energy-momentum is no longer divergence free. Instead, we have

$$
D^{\mu} T_{\mu \nu}=F \partial_{\nu} \Phi
$$

In this case,

$$
D^{\mu} J_{\mu}^{V}(\Phi)=K^{V}(\Phi)+F V^{\nu} \partial_{\nu} \Phi
$$

For the modified current,

$$
D^{\mu} J_{\mu}^{V, w}(\Phi)=K^{V, w}(\Phi)+\frac{1}{4} F w \Phi+F V^{\nu} \partial_{\nu} \Phi
$$

Proposition 5. We have

$$
\begin{gathered}
\int_{\Sigma_{\tau}} J_{\mu}^{V}(\Phi) n_{\Sigma_{\tau}}^{\mu}+\int_{\mathscr{H}\left(\tau^{\prime}, \tau\right)} J_{\mu}^{V}(\Phi) n_{\mathscr{H}^{+}\left(\tau^{\prime}, \tau\right)}^{\mu}+\iint_{\mathscr{R}\left(\tau^{\prime}, \tau\right)} K^{V}(\Phi)=\int_{\Sigma_{\tau^{\prime}}} J_{\mu}^{V}(\Phi) n_{\Sigma_{\tau^{\prime}}}^{\mu}-\iint_{\mathscr{R}\left(\tau^{\prime}, \tau\right)} F V^{\nu} \partial_{\nu} \Phi \\
\int_{\Sigma_{\tau}} J_{\mu}^{V, w^{V}}(\Phi) n_{\Sigma_{\tau}}^{\mu}+\int_{\mathscr{H}\left(\tau^{\prime}, \tau\right)} J_{\mu}^{V, w^{V}}(\Phi) n_{\mathscr{H}^{+}\left(\tau^{\prime}, \tau\right)}^{\mu}+\iint_{\mathscr{R}\left(\tau^{\prime}, \tau\right)} K^{V, w^{V}}(\Phi) \\
=\int_{\Sigma_{\tau^{\prime}}} J_{\mu}^{V, w^{V}}(\Phi) n_{\Sigma_{\tau^{\prime}}}^{\mu}+\iint_{\mathscr{R}\left(\tau^{\prime}, \tau\right)}\left(-\frac{1}{4} F w \Phi-F V^{\nu} \partial_{\nu} \Phi\right) .
\end{gathered}
$$

5.2. Vector field multipliers under metric perturbations. If we consider Kerr spacetimes such that $\epsilon$ is small, vector fields multipliers are stable if defined in the Schwarzschild coordinates $\left(t^{*}, r, x^{A}, x^{B}\right)$ or $\left(t, r \geq r_{Y}^{-}, x^{A}, x^{B}\right)$. We can consider a fixed vector field defined on the differentiable structure of a Schwarzschild exterior and compare the currents obtained using the Schwarzschild metric and the Kerr metric.

Proposition 6. Consider either the Schwarzschild $\left(t_{S}^{*}, r_{S}, x_{S}^{A}, x_{S}^{B}\right)$ coordinates or $\left(t_{S}, r_{S} \geq r_{Y}^{-}, x_{S}^{A}, x_{S}^{B}\right)$ coordinates. Suppose $V$ is a vector field defined on either of these coordinates. Then

$$
\left|\left(J_{S}^{V, w^{V}}\right)_{\mu}(\Phi) n_{\Sigma_{\tau}}^{\mu}-\left(J_{K}^{V, w^{V}}\right)_{\mu}(\Phi) n_{\Sigma_{\tau}}^{\mu}\right| \leq C \epsilon r^{-2} \max _{\alpha}\left|V^{\alpha}\right|(\partial \Phi)^{2}
$$


and

$$
\left|K_{S}^{V, w^{V}}(\Phi)-K_{K}^{V, w^{V}}(\Phi)\right| \leq C \epsilon r^{-2}\left(\left(\sum_{k=0,1} \max _{\alpha}\left|\partial^{k} V^{\alpha}\right|+|w|\right)(\partial \Phi)^{2}+\sum_{m=1,2}\left|\partial^{m} w\right| \Phi^{2}\right)
$$

\section{Statement of the main theorem}

With the currents defined, we can state our main theorem.

Main Theorem. Suppose $\square_{g_{K}} \Phi=0$. Then for all $\eta>0, R>r_{+}$and all $M>0$ there exists $a_{0}$ such that the following estimates hold in the region $\left\{r_{+} \leq r \leq R\right\}$ on Kerr spacetimes with $(M, a)$ for which $a \leq a_{0}$.

(1) Improved decay of nondegenerate energy:

$$
\begin{aligned}
& \sum_{j=0}^{\ell} \int_{\Sigma_{\tau} \cap\{r \leq R\}}\left(D^{j} \Phi\right)^{2} \\
& \quad \leq C_{R} \tau^{-3+\eta}\left(\sum_{m=0}^{\ell+2} \int_{\Sigma_{\tau_{0}}} J_{\mu}^{Z+C N, w^{Z}}\left(\partial_{t^{*}}^{m} S \Phi\right) n_{\Sigma_{\tau_{0}}}^{\mu}+\sum_{m+k+j \leq \ell+5} \int_{\Sigma_{\tau_{0}}} J_{\mu}^{Z+C N, w^{Z}}\left(\partial_{t^{*}}^{m} \hat{Y}^{k} \widetilde{\Omega}^{j} \Phi\right) n_{\Sigma_{\tau_{0}}}^{\mu}\right) .
\end{aligned}
$$

(2) Improved pointwise decay:

$$
\begin{aligned}
& \sum_{j=0}^{\ell}\left|D^{j} \Phi\right| \\
& \quad \leq C_{R} \tau^{-3 / 2+\eta}\left(\sum_{m=0}^{\ell+4} \int_{\Sigma_{\tau_{0}}} J_{\mu}^{Z+C N, w^{Z}}\left(\partial_{t^{*}}^{m} S \Phi\right) n_{\Sigma_{\tau_{0}}}^{\mu}+\sum_{m+k+j \leq \ell+7} \int_{\Sigma_{\tau_{0}}} J_{\mu}^{Z+C N, w^{Z}}\left(\partial_{t^{*}}^{m} \hat{Y^{k}} \widetilde{\Omega}^{j} \Phi\right) n_{\Sigma_{\tau_{0}}}^{\mu}\right)^{1 / 2} .
\end{aligned}
$$

Here the vector field $N$ will be defined in Section 7, and the vector field $Z$ with the modifying function $w^{Z}$ will be defined in Section 9.

Remark. We will show that although $J_{\mu}^{Z, w^{Z}}(\Phi) n_{\Sigma_{t^{*}}}^{\mu}$ is not always nonnegative, $J_{\mu}^{Z+C N, w^{Z}}(\Phi) n_{\Sigma_{t^{*}}}^{\mu}$ is nonnegative for sufficiently large $C$. Hence all the energy quantities in the theorem are nonnegative.

Remark. Since we have the improved decay of the nondegenerate energy, the theorem above can be extended beyond the event horizon. More precisely, for any $r_{b} \in\left(r_{-}, r_{+}\right)$, where $r_{-}$is the smaller root of $\Delta=r^{2}-2 M r-a^{2}$, the theorem holds up to $r \geq r_{b}$ for $D$ understood as a regular derivative inside the black hole, and with the constant depending also on $r_{b}$. The proof is similar to that in [Luk 2010].

\section{Vector field multiplier $N_{e}$ and mild growth of nondegenerate energy}

Kerr spacetime has a Killing vector field $\partial_{t}$. The conservation law gives

$$
\int_{\Sigma_{\tau}} J_{\mu}^{T}(\Phi) n_{\Sigma_{\tau}}^{\mu}+\int_{\mathscr{H}\left(\tau_{0}, \tau\right)} J_{\mu}^{T}(\Phi) n_{\Sigma_{\tau}}^{\mu}=\int_{\Sigma_{\tau_{0}}} J_{\mu}^{T}(\Phi) n_{\Sigma_{\tau_{0}}}^{\mu}+\iint_{\mathscr{R}\left(\tau_{0}, \tau\right)} \partial_{t} \Phi G .
$$

We add to the Killing vector field $\partial_{t}$ a red-shift vector field. Here, we use the "nonregular" red-shift vector field as in [Dafermos and Rodnianski 2008]. Under this construction, $N_{e}$ is $C^{0}$ but not $C^{1}$ at the 
event horizon $\mathscr{H}^{+}$. Compared to the smooth construction in [ibid.], this construction will provide extra control for some derivatives near $\mathscr{H}^{+}$.

Define

$$
Y=y_{1}(r) \hat{Y}+y_{2}(r) \hat{V}
$$

where

$$
y_{1}(r)=1-\frac{1}{\left(\log \left(r-r_{+}\right)\right)^{3}} \quad \text { and } \quad y_{2}(r)=-\frac{1}{\left(\log \left(r-r_{+}\right)\right)^{3}} .
$$

By this definition $Y$ is compactly supported in $\left\{r \leq r_{Y}^{+}\right\}$and is invariant under the isomorphisms generated by $\partial_{t^{*}}$ and $\partial_{\phi^{*}}$.

Proposition 7. Let $N_{e}=\partial_{t^{*}}+e Y$. For any $e$, there is a corresponding choice of $\epsilon \ll e$ and $r_{Y}^{-}$such that for every integer $p$, there exists $c_{p}>0$ such that

$$
\begin{aligned}
& J_{\mu}^{N_{e}}(\Phi) n_{\mathscr{H}^{+}}^{\mu} \sim\left(D_{\hat{V}} \Phi\right)^{2}+e \sum_{E_{A} \in\left\{E_{1}, E_{2}\right\}}\left(D_{E_{A}} \Phi\right)^{2} \quad \text { on the event horizon, } \\
& J_{\mu}^{N_{e}}(\Phi) n_{\Sigma_{\tau}}^{\mu} \sim \sum\left(D_{E_{\alpha}} \Phi\right)^{2}+e\left(D_{E_{\hat{Y}}} \Phi\right)^{2} \quad \text { for } r \leq r_{Y}^{-}, \\
& E_{\alpha} \in\left\{E_{1}, E_{2}, \hat{V}\right\} \\
& J_{\mu}^{N_{e}}(\Phi) n_{\Sigma_{\tau}}^{\mu} \sim \sum(\partial \Phi)^{2} \quad \text { for } r \geq r_{Y}^{-} \text {in the }\left(t^{*}, r, x^{A}, x^{B}\right) \text { coordinates, } \\
& K^{N_{e}}(\Phi) \geq c_{p} e\left(\left|\log \left(r-r_{+}\right)\right|^{p}\left(\left(D_{\hat{V}} \Phi\right)^{2}+\sum_{A}\left(D_{E_{A}} \Phi\right)^{2}\right)+\left(D_{\hat{Y}} \Phi\right)^{2}\right) \quad \text { for } r \leq r_{Y}^{-}, \\
& K^{N_{e}}(\Phi) \leq \operatorname{CeJ}_{\mu}^{N_{e}}(\Phi) n_{\Sigma_{\tau}}^{\mu} \quad \text { for } r_{Y}^{-} \leq r \leq r_{Y}^{+} .
\end{aligned}
$$

Proof. It is obvious that $Y$ is timelike and future-oriented for $r \leq r_{Y}^{-}$. Since $\partial_{t^{*}}$ is casual in the exterior region of Schwarzschild spacetime and is null only on the event horizon, for every small $e>0$, there exists sufficiently small $\epsilon>0$ such that $N_{e}$ is timelike and future-directed on Kerr spacetimes up to the event horizon. The first two estimates hold since in Kerr spacetime, $\partial_{t^{*}}$ is $\epsilon$-close to $\hat{V}$ on the event horizon. The third estimate holds because outside a small (depending on $\epsilon$ ) neighborhood of the event horizon, $\partial_{t^{*}}$ is timelike.

To show that $K^{N_{e}}(\Phi)$ has the required positivity near the event horizon, we compute the deformation tensor. First, notice that

$$
D_{\hat{Y}} y_{1}=D_{\hat{Y}} y_{2}=\frac{3 D_{\hat{Y}} r}{\left(r-r_{+}\right)\left(\log \left(r-r_{+}\right)\right)^{4}} .
$$

Using this we have

$$
\begin{aligned}
& { }^{(Y)} \pi_{\hat{V} \hat{V}}=g_{K}\left(D_{\hat{V}}\left(y_{1} \hat{Y}+y_{2} \hat{V}\right), \hat{V}\right)=-g_{K}\left(y_{1} \hat{Y}+y_{2} \hat{V}, D_{\hat{V}} \hat{V}\right)=2 y_{1} \kappa+b^{Y} y_{2}, \\
& { }^{(Y)} \pi_{\hat{Y} \hat{Y}}=g_{K}\left(D_{\hat{Y}}\left(y_{1} \hat{Y}+y_{2} \hat{V}\right), \hat{Y}\right)=-\frac{6 D_{\hat{Y}} r}{\left(r-r_{+}\right)\left(\log \left(r-r_{+}\right)\right)^{4}}, \\
& { }^{(Y)} \pi_{\hat{V} \hat{Y}}=\frac{1}{2} g_{K}\left(D_{\hat{V}}\left(y_{1} \hat{Y}+y_{2} \hat{V}\right), \hat{Y}\right)+\frac{1}{2} g_{K}\left(D_{\hat{Y}}\left(y_{1} \hat{Y}+y_{2} \hat{V}\right), \hat{V}\right)=-\frac{3 D_{\hat{Y}} r}{\left(r-r_{+}\right)\left(\log \left(r-r_{+}\right)\right)^{4}}+y_{1} \kappa+y_{2} b^{Y},
\end{aligned}
$$


Moreover, we have

$$
{ }^{(Y)} \pi_{\hat{V} E_{A}},{ }^{(Y)} \pi_{\hat{Y} E_{A}},{ }^{(Y)} \pi_{E_{A} E_{B}}=O(1)
$$

Notice that

$$
T_{\hat{Y} \hat{Y}} \sim\left(D_{\hat{Y}} \Phi\right)^{2}, \quad T_{\hat{V} \hat{V}} \sim\left(D_{\hat{V}} \Phi\right)^{2},\left.\quad T_{\hat{Y} \hat{V}} \sim|\not| \Phi\right|^{2},
$$

and that ${ }^{(Y)} \pi_{\hat{V} E_{A}},{ }^{(Y)} \pi_{\hat{Y} E_{A}}$ and ${ }^{(Y)} \pi_{E_{A} E_{B}}$ have no terms of the form $\left(D_{\hat{Y}} \Phi\right)^{2}$. Hence we can choose $r_{Y}^{-}$ sufficiently close to $r_{+}$so that for $r_{+} \leq r \leq r_{Y}^{-}$,

$$
K^{Y}(\Phi) \geq c \kappa\left(D_{\hat{Y}} \Phi\right)^{2}+\frac{c}{\left(r-r_{+}\right)\left|\log \left(r-r_{+}\right)\right|^{4}}\left(\left(D_{\hat{V}} \Phi\right)^{2}+\sum_{A}\left(D_{E_{A}} \Phi\right)^{2}\right) .
$$

Since $\partial_{t^{*}}$ is Killing, and $K^{N_{e}}(\Phi)=e K^{Y}(\Phi)$, we have

$$
K^{N_{e}}(\Phi) \geq c e\left(\kappa\left(D_{\hat{Y}} \Phi\right)^{2}+\frac{1}{\left(r-r_{+}\right)\left|\log \left(r-r_{+}\right)\right|^{4}}\left(\left(D_{\hat{V}} \Phi\right)^{2}+\sum_{A}\left(D_{E_{A}} \Phi\right)^{2}\right)\right) \quad \text { for } r \leq r_{Y}^{-}
$$

Finally, since $J_{\mu}^{\partial_{t} *} n_{\Sigma_{t^{*}}}^{\mu}$ controls all derivatives in the region $r_{Y}^{-} \leq r \leq r_{Y}^{+}$, we have

$$
K^{N_{e}}(\Phi) \leq C e J_{\mu}^{N_{e}}(\Phi) n_{\Sigma_{\tau}}^{\mu} \text { for } r_{Y}^{-} \leq r \leq r_{Y}^{+}
$$

Definition 8. We call the positive quantity $\int_{\Sigma_{\tau}} J_{\mu}^{N_{e}}(\Phi) n_{\Sigma_{\tau}}^{\mu}$ the nondegenerate energy.

The following identity determines how the nondegenerate energy changes with $\tau$.

Proposition 9. Let $\Phi$ satisfy $\square_{g_{K}} \Phi=G$. Then

$$
\begin{aligned}
\int_{\Sigma_{\tau}} J_{\mu}^{N_{e}}(\Phi) n_{\Sigma_{\tau}}^{\mu}+\int_{\mathscr{H}\left(\tau_{0}, \tau\right)} & J_{\mu}^{N_{e}}(\Phi) n_{\Sigma_{\tau}}^{\mu}+\iint_{\mathscr{R}\left(\tau_{0}, \tau\right) \cap\left\{r \leq r_{Y}^{-}\right\}} K_{\mu}^{N_{e}}(\Phi) \\
= & \int_{\Sigma_{\tau_{0}}} J_{\mu}^{N_{e}}(\Phi) n_{\Sigma_{\tau_{0}}}^{\mu}+e \iint_{\mathscr{R}\left(\tau_{0}, \tau\right) \cap\left\{r_{Y}^{-} \leq r \leq r_{Y}^{+}\right\}} K^{Y}(\Phi)+\int_{\mathscr{R}\left(\tau_{0}, \tau\right)}\left(\partial_{t^{*}} \Phi+e Y \Phi\right) G
\end{aligned}
$$

The estimates given by the vector field $N$ are sufficient to show that, modulo inhomogeneous terms, the quantity $\int_{\Sigma_{\tau}} J_{\mu}^{N_{e}}(\Phi) n_{\Sigma_{\tau}}^{\mu}$ cannot grow too much in a short time interval:

Proposition 10. Let $\Phi$ satisfy $\square_{g_{K}} \Phi=$ G. For e sufficiently small, $\epsilon \ll e$ and $0 \leq \tau-\tau^{\prime} \leq 1$, we have

$$
\int_{\Sigma_{\tau}} J_{\mu}^{N_{e}}(\Phi) n_{\Sigma_{\tau}}^{\mu}+\int_{\mathscr{H}\left(\tau^{\prime}, \tau\right)} J_{\mu}^{N_{e}}(\Phi) n_{\mathscr{H}^{+}}^{\mu} \leq 4 \int_{\Sigma_{\tau^{\prime}}} J_{\mu}^{N_{e}}(\Phi) n_{\Sigma_{\tau^{\prime}}}^{\mu}+C \iint_{\mathscr{R}\left(\tau^{\prime}, \tau\right)} G^{2} .
$$

Proof. We first note that

$$
\iint_{\mathscr{R}\left(\tau^{\prime}, \tau\right) \cap\left\{r_{Y}^{-} \leq r \leq r_{Y}^{+}\right\}} K^{Y}(\Phi) \leq C \int_{\tau^{\prime}}^{\tau} \int_{\Sigma_{\bar{\tau}}} J_{\mu}^{N_{e}}(\Phi) n_{\Sigma_{\bar{\tau}}}^{\mu} d \bar{\tau}
$$


with $C$ independent of $e$ and $\epsilon$ whenever $\epsilon \ll e<1$. Then, by Proposition 9,

$$
\begin{aligned}
\int_{\Sigma_{\tau}} & J_{\mu}^{N_{e}}(\Phi) n_{\Sigma_{\tau}}^{\mu}+\int_{\mathscr{H}\left(\tau^{\prime}, \tau\right)} J_{\mu}^{N_{e}}(\Phi) n_{\mathscr{H}^{+}}^{\mu}+\iint_{\mathscr{R}\left(\tau^{\prime}, \tau\right) \cap\left\{r \leq r_{Y}^{-}\right\}} K_{\mu}^{N_{e}}(\Phi) \\
= & \int_{\Sigma_{\tau^{\prime}}} J_{\mu}^{N_{e}}(\Phi) n_{\Sigma_{\tau^{\prime}}}^{\mu}+e \iint_{\mathscr{R}\left(\tau^{\prime}, \tau\right) \cap\left\{r_{Y}^{-} \leq r \leq r_{Y}^{+}\right\}} K^{Y}(\Phi)+\int_{\mathscr{R}\left(\tau^{\prime}, \tau\right)}\left(\partial_{t^{*}} \Phi+e Y \Phi\right) G \\
\leq & \int_{\Sigma_{\tau^{\prime}}} J_{\mu}^{N_{e}}(\Phi) n_{\Sigma_{\tau^{\prime}}}^{\mu}+C e \int_{\tau^{\prime}}^{\tau} \int_{\Sigma_{\bar{\tau}}} J_{\mu}^{N_{e}}(\Phi) n_{\Sigma_{\bar{\tau}}}^{\mu} d \bar{\tau}+\delta^{\prime} \iint_{\mathscr{R}\left(\tau^{\prime}, \tau\right)}\left(\left(\partial_{t^{*}} \Phi+e Y \Phi\right)\right)^{2}+\left(\delta^{\prime}\right)^{-1} \iint_{\mathscr{R}\left(\tau^{\prime}, \tau\right)} G^{2} \\
\leq & \int_{\Sigma_{\tau^{\prime}}} J_{\mu}^{N_{e}}(\Phi) n_{\Sigma_{\tau^{\prime}}}^{\mu}+\left(C \delta^{\prime}+2 C e\right) \int_{\tau^{\prime}}^{\tau} \int_{\Sigma_{\bar{\tau}}} J_{\mu}^{N_{e}}(\Phi) n_{\Sigma_{\bar{\tau}}}^{\mu} d \bar{\tau}+\left(\delta^{\prime}\right)^{-1} \iint_{\mathscr{R}\left(\tau^{\prime}, \tau\right)} G^{2} .
\end{aligned}
$$

By Gronwall's inequality and absorbing $\left(\delta^{\prime}\right)^{-1}$ into the constant $C$, we have

$$
\int_{\Sigma_{\tau}} J_{\mu}^{N_{e}}(\Phi) n_{\Sigma_{\tau}}^{\mu} \leq 2 \int_{\Sigma_{\tau^{\prime}}} J_{\mu}^{N_{e}}(\Phi) n_{\Sigma_{\tau^{\prime}}}^{\mu}+C \iint_{\mathscr{R}\left(\tau^{\prime}, \tau\right)} G^{2} .
$$

Now the estimate for the term horizon follows from Proposition 9.

\section{Integrated decay estimates and boundedness of nondegenerate energy}

In this section we would like to show an integrated decay estimate. We first follow [Luk 2010] to construct a vector field and prove an integrated decay estimate for the terms near spatial infinity. That construction is in turn inspired by [Sterbenz 2005]. In [Luk 2010], the decay rate in $r$ of this integrated decay estimate is crucial for controlling the error terms arising from the vector field commutator $S$. In the sequel, such an estimate will also facilitate many computations as we prove the full integrated decay estimate.

In view of the red shift, all derivatives of $\Phi$ can be controlled near the event horizon. However, we would also like to prove an integrated decay estimate that controls $\Phi$ itself near the event horizon. This is in contrast to the integrated decay estimate in [Dafermos and Rodnianski 2008], which degenerates near the event horizon. This extra control is useful as we are considering the inhomogeneous problem.

The proof of the integrated decay estimate for a finite region of $r$ away from the horizon follows that in [Dafermos and Rodnianski 2008]. The one difference here is that we do not assume the boundedness of $\int_{\Sigma_{\tau}} J_{\mu}^{N_{e}}(\Phi) n_{\Sigma_{\tau}}^{\mu}$ (even after ignoring inhomogeneous terms). We would instead like to prove the boundedness of $\int_{\Sigma_{\tau}} J_{\mu}^{N_{e}}(\Phi) n_{\Sigma_{\tau}}^{\mu}$ using the integrated decay estimates. We will, however, use Proposition 10.

The reader should think of this integrated decay estimates as analogous to the estimates associated to the vector field $X$ in [Dafermos and Rodnianski 2009; 2011; Luk 2010]. However, it is impossible to obtain such estimates using a vector field in Kerr spacetimes and we therefore resort to a phase space analysis; see [Alinhac 2009].

To perform the phase space analysis, we will take the Fourier transform in the variable $t^{*}$, take the Fourier series in the variable $\phi^{*}$ and express the dependence on the $\theta$ variable in oblate spheroidal harmonics. Carter [1968] discovered that with this decomposition, the wave equation can be separated. 
However, to take the Fourier transform in the variable $t^{*}$, we need $\Phi$ to be at least in $L^{2}$. To this end, we perform a cutoff in the variable $t^{*}$.

8.1. Estimates near spatial infinity. In this subsection, we follow [Luk 2010] to construct a vector field $\tilde{X}=\tilde{f}\left(r^{*}\right) \partial_{r^{*}}$ such that the spacetime integral that can be controlled with a good weight in $r$.

Proposition 11 [Luk 2010, Proposition 8]. In Schwarzschild spacetimes, using $\left(t, r^{*}, x^{A}, x^{B}\right)$ coordinates, there exists $\tilde{X}_{S}=\tilde{f}\left(r^{*}\right) \partial_{r^{*}}$ and $w_{S}^{\tilde{X}}$ supported in $r \geq \frac{13}{4} M$, such that

$$
K^{\tilde{X}, w^{\tilde{X}}}(\Phi) \geq c\left(r^{-1-\delta}\left(\partial_{r^{*}} \Phi\right)^{2}+\left.r^{-1}|\not| \Phi\right|^{2}+r^{-3-\delta} \Phi^{2}\right) \quad \text { for } r^{*} \geq \max \{100,100 M\}
$$

and

$$
\left|\int_{\Sigma_{\tau}} J_{\mu}^{\tilde{X}, w^{\tilde{X}}}(\Phi) n_{\Sigma_{\tau}}^{\mu}\right| \leq C \int_{\Sigma_{\tau}} J_{\mu}^{N_{e}}(\Phi) n_{\Sigma_{\tau}}^{\mu} .
$$

This implies via stability (since the vector field is supported away from the event horizon) the following: Proposition 12. In Kerr spacetimes, using $\left(t^{*}, r, x^{A}, x^{B}\right)$ coordinates, there exists $\tilde{X}$ and $w^{\tilde{X}}$ supported in $r \geq \frac{25}{8} M$ such that for some large $R$,

$$
K^{\tilde{X}, w^{\tilde{X}}}(\Phi) \geq c_{\tilde{X}}\left(r^{-1-\delta}\left(\partial_{r^{*}} \Phi\right)^{2}+\left.r^{-1}|\not| \Phi\right|^{2}+r^{-3-\delta} \Phi^{2}\right)-C_{\tilde{X}} \epsilon r^{-2}\left(\partial_{t^{*}} \Phi\right)^{2} \quad \text { for } r^{*} \geq R
$$

and

$$
\left|\int_{\Sigma_{\tau}} J_{\mu}^{\tilde{X}, w^{\tilde{X}}}(\Phi) n_{\Sigma_{\tau}}^{\mu}\right| \leq C \int_{\Sigma_{\tau}} J_{\mu}^{N_{e}}(\Phi) n_{\Sigma_{\tau}}^{\mu} .
$$

Now it is easy to construct the following vector field on Schwarzschild spacetimes:

Proposition 13. In Schwarzschild spacetimes, in $\left(t, r^{*}, x^{A}, x^{B}\right)$ coordinates, there exists $\check{X}_{S}=\check{f}\left(r^{*}\right) \partial_{r^{*}}$ supported in $r \geq \frac{13}{4} M$ such that

$$
K^{\check{X}}(\Phi) \geq c r^{-1-\delta}\left(\partial_{t^{*}} \Phi\right)^{2}-C\left(r^{-1-\delta}\left(\partial_{r^{*}} \Phi\right)^{2}+\left.r^{-1}|\not| \Phi\right|^{2}+r^{-3-\delta} \Phi^{2}\right) \quad \text { for } r^{*} \geq \max \{100,100 M\}
$$

and

$$
\left|\int_{\Sigma_{\tau}} J_{\mu}^{\check{X}}(\Phi) n_{\Sigma_{\tau}}^{\mu}\right| \leq C \int_{\Sigma_{\tau}} J_{\mu}^{N_{e}}(\Phi) n_{\Sigma_{\tau}}^{\mu} .
$$

Proof. Let $\check{f}$ be supported appropriately and let $\check{f}\left(r^{*}\right)=1 /\left(1+r^{*}\right)^{\delta}$ whenever $r^{*}$ is large.

As before, a stability argument gives this:

Proposition 14. In Kerr spacetimes, in $\left(t^{*}, r, x^{A}, x^{B}\right)$ coordinates, there exists $\check{X}$ supported in $r \geq \frac{25}{8} M$ such that for some large $R$,

$$
K^{\check{X}}(\Phi) \geq c r^{-1-\delta}\left(\partial_{t^{*}} \Phi\right)^{2}-C_{\check{X}}\left(r^{-1-\delta}\left(\partial_{r^{*}} \Phi\right)^{2}+\left.r^{-1}|\not| \Phi\right|^{2}+r^{-3-\delta} \Phi^{2}\right) \quad \text { for } r^{*} \geq R
$$

and

$$
\left|\int_{\Sigma_{\tau}} J_{\mu}^{\check{X}}(\Phi) n_{\Sigma_{\tau}}^{\mu}\right| \leq C \int_{\Sigma_{\tau}} J_{\mu}^{N_{e}}(\Phi) n_{\Sigma_{\tau}}^{\mu} .
$$

Now using the vector field $\tilde{X}+\frac{1}{2}\left(c_{\tilde{X}} / C_{\check{X}}\right) \check{X}$ and modifying function $w^{\tilde{X}}$, we get the following estimate: 
Proposition 15. For $\epsilon$ sufficiently small,

$$
\begin{aligned}
& \iint_{\mathscr{R}\left(\tau_{0}, \tau\right) \cap\{r \geq R\}}\left(r^{-1-\delta} J_{\mu}^{N_{e}}(\Phi) n_{\Sigma_{t^{*}}}^{\mu}+r^{-3-\delta} \Phi^{2}\right) \\
& \quad \leq C\left(\int_{\Sigma_{\tau}} J_{\mu}^{N_{e}}(\Phi) n_{\Sigma_{\tau}}^{\mu}+\iint_{\Sigma_{\tau_{0}}} J_{\mu}^{N}(\Phi) n_{\Sigma_{\tau_{0}}}^{\mu}+\iint_{\mathscr{R}\left(\tau_{0}, \tau\right) \cap\left\{\frac{25}{8} M \leq r \leq R\right\}}\left(J_{\mu}^{N_{e}}(\Phi) n_{\Sigma_{t^{*}}}^{\mu}+\Phi^{2}\right)+\iint_{\mathscr{R}\left(\tau_{0}, \tau\right)}\left(\left|\partial_{r} \Phi\right|+r^{-1}|\Phi|\right)|G|\right) .
\end{aligned}
$$

8.2. Estimates near the event horizon. The integrated decay estimates shown in [Dafermos and Rodnianski 2008] are degenerate around the event horizon. Here we will prove the corresponding estimates near the event horizon. In view of the availability of the red-shift estimate $K^{N_{e}}$, we will focus on the zeroth order term $\Phi^{2}$. It turns out that we can use a construction in [Luk 2010].

Proposition 16. In Schwarzschild spacetimes, in $\left(t, r^{*}, x^{A}, x^{B}\right)$ coordinates, there exists $X_{h}=f_{h}\left(r^{*}\right) \partial_{r^{*}}$ and $w^{X_{h}}$ supported in $r \leq \frac{23}{8} M$ such that

$$
K^{X_{h}, w^{X_{h}}}(\Phi) \geq c\left(\left(\partial_{r^{*}} \Phi\right)^{2}+\left.|\not| \Phi\right|^{2}+\Phi^{2}\right) \quad \text { for } r \leq r_{Y}^{-}
$$

and

$$
\left|\int_{\Sigma_{\tau}} J_{\mu}^{X_{h}, w^{X_{h}}}(\Phi) n_{\Sigma_{\tau}}^{\mu}\right| \leq C \int_{\Sigma_{\tau}} J_{\mu}^{N_{e}}(\Phi) n_{\Sigma_{\tau}}^{\mu} \quad \text { and } \quad\left|J_{\mu}^{X_{h}, w^{X_{h}}}(\Phi) n_{\mathscr{H}^{+}}^{\mu}\right| \leq C J_{\mu}^{N_{e}}(\Phi) n_{\mathscr{H}^{+}}^{\mu} .
$$

Proof. Let

$$
X_{h}=f_{h}\left(r_{S}^{*}\right) \partial_{r_{S}^{*}}=-\chi(r) \frac{M^{3}}{\left(1+4 \mu^{-2}\right)} \partial_{r_{S}^{*}}=-\chi(r) \frac{\mu^{3} r^{3}}{8\left(1+4 \mu^{-2}\right)} \partial_{r_{S}^{*}}
$$

where $\chi(r)$ is a cutoff function that is compactly supported in $r \leq \frac{23}{8} M$ and is identically 1 for $r \leq r_{Y}^{-}$. Also, let

$$
w^{X_{h}}=2 f_{h}^{\prime}\left(r^{*}\right)+\frac{4(1-\mu)}{r} f_{h}\left(r^{*}\right)
$$

From now on, we will focus on the behavior when $r \leq r_{Y}^{-}$and treat the terms in $\left\{r_{Y}^{-} \leq r \leq \frac{23}{8} M\right\}$ as errors. Recall that on Schwarzschild spacetime,

$$
\begin{aligned}
K^{X_{h}, w^{X_{h}}}(\Phi)=\frac{f^{\prime}\left(r^{*}\right)}{1-\mu}\left(\partial_{r^{*}} \Phi\right)^{2}+ & \frac{(2-3 \mu) f\left(r^{*}\right)}{2 r}|\not \Phi|^{2} \\
& -\frac{1}{4}\left(\frac{1}{1-\mu} f^{\prime \prime \prime}\left(r^{*}\right)+\frac{4}{r} f^{\prime \prime}\left(r^{*}\right)+\frac{\mu}{r^{2}} f^{\prime}\left(r^{*}\right)-\frac{2 \mu}{r^{3}}(3-4 \mu) f\left(r^{*}\right)\right) \Phi^{2} .
\end{aligned}
$$

We now look at the sign of this expression for $r \leq r_{Y}^{-}$. It is easy to see that the coefficient for $\left(\partial_{r^{*}} \Phi\right)^{2}$ is positive:

$$
f^{\prime}\left(r^{*}\right)=(1-\mu) \partial_{r} f_{0}(r)=\frac{\mu r^{2}(1-\mu)}{\left(1+4 \mu^{-2}\right)^{2}} \geq \frac{c(1-\mu)}{r^{3}}
$$


The coefficient of $\left.|\not| \Phi\right|^{2}$ is also clearly positive. A computation shows that

$$
\begin{aligned}
\frac{1}{1-\mu} f^{\prime \prime \prime}+\frac{4}{r} f^{\prime \prime}+\frac{\mu}{r^{2}} & f^{\prime}-\frac{2 \mu}{r^{3}}(3-4 \mu) f \\
& =-\frac{\mu^{6}(192+\mu(128+\mu(-784+\mu(464+\mu(-28+\mu(52+\mu(-3+4 \mu)))))))}{4\left(4+\mu^{2}\right)^{4}}
\end{aligned}
$$

We want to show that $P(\mu)=192+\mu(128+\mu(-784+\mu(464+\mu(-28+\mu(52+\mu(-3+4 \mu)))))) \geq 1 / 7$ for $16 / 23 \leq \mu \leq 1$.

First, $192+128 \mu-784 \mu^{2}+464 \mu^{3}=16\left(-12-20 \mu+29 \mu^{2}\right)(\mu-1) \geq 0$.

Now $52-3 \mu+4 \mu^{2}$ reaches its minimum at $3 / 8$. Hence, $52-3 \mu+4 \mu^{2} \geq 823 / 16$.

Finally $-28+\mu\left(52-3 \mu+4 \mu^{2}\right) \geq-28+11 / 20 \cdot 823 / 16 \geq 93 / 320$.

Therefore, $P(\mu) \geq 1023 / 6400 \geq 1 / 7$ for $16 / 23 \leq \mu \leq 1$. Therefore, for $r \leq r_{Y}^{-}$,

$$
K^{X_{h}, w^{X_{h}}}(\Phi) \geq c\left(\left(\partial_{r^{*}} \Phi\right)^{2}+\left.|\not| \Phi\right|^{2}+\Phi^{2}\right) .
$$

The second and third statements,

$$
\left|\int_{\Sigma_{\tau}} J_{\mu}^{X_{h}, w^{X_{h}}}(\Phi) n_{\Sigma_{\tau}}^{\mu}\right| \leq C \int_{\Sigma_{\tau}} J_{\mu}^{N_{e}}(\Phi) n_{\Sigma_{\tau}}^{\mu} \quad \text { and } \quad\left|J_{\mu}^{X_{h}, w^{X_{h}}}(\Phi) n_{\mathscr{H}^{+}}^{\mu}\right| \leq C J_{\mu}^{N_{e}}(\Phi) n_{\mathscr{H}^{+}}^{\mu},
$$

follow from the boundedness of $f_{h}$ and $w^{X_{h}}$ and that on the Schwarzschild horizon $\partial_{t}=\partial_{r^{*}}$. Hence in both estimates, the constants are independent of $e$ for $e$ small.

Because $X_{h}$ and $w^{X_{h}}$ are actually smooth up to the event horizon, we have this via a stability argument:

Proposition 17. In Kerr spacetimes, using $\left(t_{S}, r_{S}, x_{S}^{1}, x_{S}^{2}\right)$ coordinates, there exists $X_{h}$ and $w^{X_{h}}$ supported in $r \leq \frac{23}{8} M$ such that

$$
K^{X_{h}, w^{X_{h}}}(\Phi) \geq c \Phi^{2}-C \epsilon\left(\partial_{t^{*}} \Phi\right)^{2}-C \epsilon\left(\partial_{r} \Phi\right)^{2} \quad \text { for } r \leq r_{Y}^{-}
$$

and

$$
\left|\int_{\Sigma_{\tau}} J_{\mu}^{X_{h}, w^{X_{h}}}(\Phi) n_{\Sigma_{\tau}}^{\mu}\right| \leq C \int_{\Sigma_{\tau}} J_{\mu}^{N}(\Phi) n_{\Sigma_{\tau}}^{\mu} \quad \text { and } \quad\left|J_{\mu}^{X_{h}, w^{X_{h}}}(\Phi) n_{\mathscr{H}^{+}}^{\mu}\right| \leq C J_{\mu}^{N_{e}}(\Phi) n_{\mathscr{H}^{+}}^{\mu} .
$$

Together with the red shift, we then have an integrated decay estimate near the event horizon:

\section{Proposition 18.}

$$
\begin{aligned}
\iint_{\mathscr{R}\left(\tau_{0}, \tau\right) \cap\left\{r \leq r_{Y}^{-}\right\}}\left(\Phi^{2}+K^{N_{e}}(\Phi)\right) \\
\leq C\left(\int_{\Sigma_{\tau}} J_{\mu}^{N_{e}}(\Phi) n_{\Sigma_{\tau}}^{\mu}+\int_{\Sigma_{\tau_{0}}} J_{\mu}^{N_{e}}(\Phi) n_{\Sigma_{\tau_{0}}}^{\mu}+\iint_{\mathscr{R}\left(\tau_{0}, \tau\right) \cap\left\{r_{Y}^{-} \leq r \leq \frac{23}{8} M\right\}}\left(\Phi^{2}+J_{\mu}^{N_{e}}(\Phi) n_{\Sigma_{t^{*}}}^{\mu}\right)\right. \\
\left.\quad \quad f \int_{\mathscr{R}\left(\tau_{0}, \tau\right) \cap\left\{r \leq \frac{23}{8} M\right\}}\left(\left|\partial_{r^{*}} \Phi\right|+r^{-1}|\Phi|\right)|G|+\left|\iint_{\mathscr{R}\left(\tau_{0}, \tau\right) \cap\left\{r \leq \frac{23}{8} M\right\}}\left(\partial_{t^{*}} \Phi+e Y \Phi\right) G\right|\right) .
\end{aligned}
$$


8.3. Cutoff, decomposition and separation. Following [Dafermos and Rodnianski 2008], we define the cutoff $\Phi_{\tau^{\prime}}^{\tau}=\xi \Phi$, where $\xi=\chi\left(t^{*}-1-\tau\right) \chi\left(-t^{*}-1+\tau^{\prime}\right)$, for some smooth cutoff function $\chi(x)$ that is identically 1 for $x \leq-1$ and has support on $\{x \leq 0\}$. Then

$$
\square_{g} \Phi_{\tau^{\prime}}^{\tau}=\xi G+2 D^{\alpha} \Phi D_{\alpha} \xi+\Phi \square_{g} \xi=: F
$$

We then decompose in frequency. We decompose the Fourier transform in $t$ of $\Phi$ into Fourier series in $\phi$ and oblate spheroidal harmonics:

$$
\hat{\Phi}_{\tau^{\prime}}^{\tau}=\sum_{m, \ell} R_{m \ell}^{\omega}(r) S_{m \ell}(a \omega, \cos \theta) e^{i m \phi} .
$$

We also decompose the inhomogeneous term $F$ (which comes both from the original inhomogeneous term $G$ and the cutoff):

$$
\hat{F}=\sum_{m, \ell} F_{m \ell}^{\omega}(r) S_{m \ell}(a \omega, \cos \theta) e^{i m \phi} .
$$

Letting $\zeta$ be a sharp cutoff with such that $\zeta=1$ for $|x| \leq 1$ and $\zeta=0$ for $|x|>1$, we define

$$
\begin{aligned}
& \Phi_{b}=\int_{-\infty}^{\infty} \zeta\left(\omega / \omega_{1}\right) \sum_{m, l: \lambda_{m l}(\omega) \leq \lambda_{1}} R_{m l}^{\omega}(r) S_{m l}(a \omega, \cos \theta) e^{i m \phi} e^{i \omega t} d \omega, \\
& \Phi_{\downarrow}=\int_{-\infty}^{\infty} \zeta\left(\omega / \omega_{1}\right) \sum_{m, l: \lambda_{m l}(\omega)>\lambda_{1}} R_{m l}^{\omega}(r) S_{m l}(a \omega, \cos \theta) e^{i m \phi} e^{i \omega t} d \omega, \\
& \Phi_{\natural}=\int_{-\infty}^{\infty}\left(1-\zeta\left(\omega / \omega_{1}\right)\right) \sum_{m, l: \lambda_{m l}(\omega) \geq \lambda_{2} \omega^{2}} R_{m l}^{\omega}(r) S_{m l}(a \omega, \cos \theta) e^{i m \phi} e^{i \omega t} d \omega, \\
& \Phi_{\sharp}=\int_{-\infty}^{\infty}\left(1-\zeta\left(\omega / \omega_{1}\right)\right) \sum_{m, l: \lambda_{m l}(\omega)<\lambda_{2} \omega^{2}} R_{m l}^{\omega}(r) S_{m l}(a \omega, \cos \theta) e^{i m \phi} e^{i \omega t} d \omega .
\end{aligned}
$$

In this decomposition, we think of $\omega_{1}$ as large and $\lambda_{2}$ as small.

8.4. The trapped frequencies. Trapping occurs for $\Phi_{\natural}$. An integrated decay estimate is proved in detail in [Dafermos and Rodnianski 2008, Section 5.3.3]. The first term on right side in the following proposition is different from that in [ibid.], but the inequality still holds as a result of the proof of the corresponding inequality there.

\section{Proposition 19.}

$$
\begin{aligned}
& \iint_{\mathscr{R}(-\infty, \infty)}\left(\chi \Phi_{\natural}^{2}+\chi\left(\partial_{r} \Phi_{\natural}\right)^{2}+\chi \mathbb{1}_{\left\{|r-3 M| \geq \frac{1}{8} M\right\}} J_{\mu}^{N}\left(\Phi_{\sharp}\right) n_{\Sigma_{t^{*}}}^{\mu}\right) \\
& \leq C \int_{\mathscr{H}(-\infty, \infty)}\left(\partial_{t^{*}} \Phi_{\tau^{\prime}}^{\tau}\right)^{2}+C \epsilon \int_{\mathscr{H}(-\infty, \infty)}\left(\partial_{\phi^{*}} \Phi_{\tau^{\prime}}^{\tau}\right)^{2}+\int_{-\infty}^{\infty} d t^{*} \int_{r \geq R}\left(2 f\left(r^{2}+a^{2}\right)^{1 / 2} F_{\natural} \partial_{r^{*}}\left(\left(r^{2}+a^{2}\right)^{1 / 2} \Phi_{\tau^{\prime}}^{\tau}\right)\right. \\
& \left.\quad+f^{\prime}\left(r^{2}+a^{2}\right) F_{\sharp} \Phi_{\tau^{\prime}}^{\tau}\right) \frac{\Delta}{r^{2}+a^{2}} \sin \theta d \phi d \theta d r^{*}+\delta^{\prime} \iint_{\mathscr{R} \cap\{r \leq R\}}\left(\Phi_{\tau^{\prime}}^{\tau}\right)^{2}+\left(\partial_{r^{*}} \Phi_{\tau^{\prime}}^{\tau}\right)^{2}+C\left(\delta^{\prime}\right)^{-1} \iint_{\mathscr{R} \cap\{r \leq R\}} F^{2},
\end{aligned}
$$


where $\chi$ is a weight that degenerates at infinity and near the event horizon and $f$ is increasing and $f=\tan ^{-1}\left(r^{*}-\alpha-\sqrt{\alpha}\right) / \alpha-\tan ^{-1}(-1-\alpha)^{-1 / 2}$ for $r>R$ for some fixed $\alpha$.

8.5. The untrapped frequencies. For each of the pieces that are untrapped, that is, $\Phi_{\bullet}$ for $\bullet=b, \mathrm{~d}$ or $\sharp$, Dafermos and Rodnianski [2008] constructed a vector field $X_{\bullet}$ such that

$$
\iint_{\mathscr{R}^{(-\infty, \infty)}} \chi\left(J_{\mu}^{N_{e}}\left(\Phi_{\bullet}\right) n_{\Sigma_{t^{*}}}^{\mu}+\Phi_{\bullet}^{2}\right) \leq C \iint_{\mathscr{R}(-\infty, \infty)} K^{X_{\bullet}}\left(\Phi_{\bullet}\right),
$$

where $\chi$ is a weight function that both degenerates at infinity and vanishes around the event horizon. Using this vector field and the conservation identity, they showed the following:

\section{Proposition 20.}

$$
\begin{gathered}
\iint_{\mathscr{R}(-\infty, \infty)} \chi\left(\left(J_{\mu}^{N_{e}}\left(\Phi_{\mathrm{b}}\right)+J_{\mu}^{N_{e}}\left(\Phi_{\mathrm{d}}\right)+J_{\mu}^{N}\left(\Phi_{\sharp}\right)\right) n_{\Sigma_{\tau}}^{\mu}+\left(\Phi_{b}^{2}+\Phi_{\mathrm{d}}^{2}+\Phi_{\sharp}^{2}\right)\right) \\
\leq C \int_{\mathscr{H}(-\infty, \infty)} J_{\mu}^{N_{e}}(\Phi) n_{\mathscr{H} \mathcal{H}^{+}}^{\mu}+C\left(\delta^{\prime}\right)^{-1} \iint_{\mathscr{R}(-\infty, \infty) \cap\{r \leq R\}} F^{2} \\
+C \delta^{\prime} \iint_{\mathscr{R}^{\prime}(-\infty, \infty) \cap\{r \leq R\}}\left(\Phi_{\tau^{\prime}}^{\tau}\right)^{2}+\left(\partial_{r^{*}} \Phi_{\tau^{\prime}}^{\tau}\right)^{2}+\mathbb{1}_{\left\{r \leq \frac{23}{8} M\right\}} J_{\mu}^{N}\left(\Phi_{\tau^{\prime}}^{\tau}\right) n_{\Sigma_{t^{*}}}^{\mu} \\
+\int_{-\infty}^{\infty} d t^{*} \int_{\{r \geq R\}}\left(2 f\left(r^{2}+a^{2}\right)^{1 / 2}\left(F_{\mathrm{b}}+F_{\mathrm{d}}+F_{\sharp}\right) \partial_{r^{*}}\left(\left(r^{2}+a^{2}\right)^{1 / 2} \Phi_{\tau^{\prime}}^{\tau}\right)\right. \\
\left.+f^{\prime}\left(r^{2}+a^{2}\right)\left(F_{\mathrm{b}}+F_{\mathrm{d}}+F_{\sharp}\right) \Phi_{\tau^{\prime}}^{\tau}\right) \frac{\Delta}{r^{2}+a^{2}} \sin \theta d \phi d \theta d r^{*},
\end{gathered}
$$

where $\chi$ and $f$ are exactly as in Proposition 19.

Proof. This inequality is essentially borrowed from Dafermos and Rodnianski [2008, Section 5.3.4]. The only difference is the first term on it right side. They used the estimate

$$
\int_{\mathscr{H}(-\infty, \infty)} J_{\mu}^{X \cdot}\left(\Phi_{\bullet}\right) n_{\mathscr{H}^{+}}^{\mu} \leq C \int_{\Sigma_{\tau^{\prime}}} J_{\mu}^{N}\left(\Phi_{\tau^{\prime}}^{\tau}\right) n_{\Sigma_{\tau^{\prime}}}^{\mu} .
$$

Here, we have not proved boundedness of the solution and hence we are content with the estimate

$$
\int_{\mathscr{H}(-\infty, \infty)} J_{\mu}^{X \cdot}\left(\Phi_{\bullet}\right) n_{\mathscr{H}^{+}}^{\mu} \leq C \int_{\mathscr{H}(-\infty, \infty)} J_{\mu}^{N_{e}}\left(\Phi_{\tau^{\prime}}^{\tau}\right) n_{\mathscr{H}^{+}}^{\mu} .
$$

This estimate holds for $C$ independent of $e$ because $X_{\bullet}$ is constructed as $f \partial_{r^{*}}$ and, on the event horizon, $\partial_{r^{*}}=O(1) \hat{V}+O(\epsilon) E_{A}$.

8.6. The integrated decay estimates. To add up the estimates in the previous sections, we need a Hardytype inequality:

Proposition 21. For $R^{\prime}<R$,

$$
\int_{\Sigma_{\tau} \cap\{r \geq R\}} r^{\alpha-2} \Phi^{2} \leq C \int_{\Sigma_{\tau} \cap\left\{r \geq R^{\prime}\right\}} r^{\alpha} J_{\mu}^{N_{e}}(\Phi) n_{\Sigma_{\tau}}^{\mu} .
$$


Proof. Let $k(r)$ be defined by solving $k^{\prime}(r, \theta, \phi)=r^{\alpha-2} \operatorname{vol}$, where $\operatorname{vol}=\operatorname{vol}(r, \theta, \phi)$ is the volume density on $\Sigma_{\tau}$ with $r, \theta, \phi$ coordinates, with boundary condition $k\left(R^{\prime}, \theta, \phi\right)=0$. Now

$$
\begin{aligned}
\int_{\Sigma_{\tau}} r^{\alpha-2} \Phi^{2} & =\iiint_{r_{+}}^{\infty} k^{\prime}(r) \Phi^{2} d r d \theta d \phi \\
& =-2 \iiint k(r) \Phi \partial_{r} \Phi d r d \theta d \phi \\
& \leq 2\left(\iiint \frac{k(r)^{2}}{k^{\prime}(r)}\left(\partial_{r} \Phi\right)^{2} d r d \theta d \phi\right)^{1 / 2}\left(\iiint k^{\prime}(r) \Phi^{2} d r d \theta d \phi\right)^{1 / 2} .
\end{aligned}
$$

Since vol $\sim r^{2}, k(r) \sim r^{\alpha+1}$ and $k^{\prime}(r) \sim r^{\alpha}$, we have $\left(1+k(r)^{2}\right) /\left(1+k^{\prime}(r)\right) \sim r^{\alpha}$ vol.

We now add up the estimates for $\Phi_{b}, \Phi_{\downarrow}, \Phi_{\sharp}$ and $\Phi_{\sharp}$.

\section{Proposition 22.}

$$
\begin{aligned}
& \iint_{\mathscr{R}\left(\tau^{\prime}, \tau\right)}\left(r^{-1-\delta} \mathbb{1}_{\left\{|r-3 M| \geq \frac{1}{8} M\right\}} J_{\mu}^{N_{e}}(\Phi) n_{\Sigma_{\tau}}^{\mu}+r^{-1-\delta}\left(\partial_{r} \Phi\right)^{2}+r^{-3-\delta} \Phi^{2}\right) \\
& \leq C\left(\int_{\Sigma_{\tau}} J_{\mu}^{N_{e}}(\Phi) n_{\Sigma_{\tau}}^{\mu}+\int_{\Sigma_{\tau^{\prime}}} J_{\mu}^{N_{e}}(\Phi) n_{\Sigma_{\tau^{\prime}}}^{\mu}+\int_{\mathscr{H}\left(\tau^{\prime}, \tau\right)} J_{\mu}^{N_{e}}(\Phi) n_{\mathscr{H} \mathcal{H}^{+}}^{\mu}\right. \\
& \quad+\iint_{\mathscr{R}\left(\tau^{\prime}-1, \tau+1\right)}\left(\left|\partial_{r^{*}} \Phi\right|+r^{-1}|\Phi|\right)|G|+\left|\iint_{\mathscr{R}\left(\tau^{\prime}-1, \tau+1\right) \cap\left\{r \leq \frac{23}{8} M\right\}}\left(\partial_{t^{*}} \Phi+e Y \Phi\right) G\right| \\
& \left.+\iint_{\mathscr{R}\left(\tau^{\prime}-1, \tau+1\right)} G^{2}\right) .
\end{aligned}
$$

Proof. Since the function $f$ appears identically in Propositions 19 and 20, we can add up the estimates to obtain

$$
\begin{gathered}
\iint_{\mathscr{R}(-\infty, \infty)}\left(\chi\left(J_{\mu}^{N_{e}}\left(\Phi_{b}\right)+J_{\mu}^{N_{e}}\left(\Phi_{\mathrm{d}}\right)+\mathbb{1}_{\left\{|r-3 M| \geq \frac{1}{8} M\right\}} J_{\mu}^{N_{e}}\left(\Phi_{\sharp}\right)+J_{\mu}^{N_{e}}\left(\Phi_{\sharp}\right)\right) n_{\Sigma_{\tau}}^{\mu}+\chi\left(\Phi_{b}^{2}+\Phi_{\mathrm{d}}^{2}+\Phi_{\sharp}^{2}+\Phi_{\sharp}^{2}\right)\right) \\
\leq C \int_{\mathscr{H}\left(\tau^{\prime}-1, \tau+1\right)} J_{\mu}^{N_{e}}(\Phi) n_{\mathscr{\ell ^ { + }}}^{\mu}+C\left(\delta^{\prime}\right)^{-1} \iint_{\mathscr{R}(-\infty, \infty) \cap\{r \leq R\}} F^{2} \\
\quad+C \delta^{\prime} \iint_{\mathscr{R}^{\prime}(-\infty, \infty) \cap\{r \leq R\}}\left(\Phi_{\tau^{\prime}}^{\tau}\right)^{2}+\left(\partial_{r^{*}} \Phi_{\tau^{\prime}}^{\tau}\right)^{2}+\mathbb{1}_{\left\{r \leq \frac{23}{8} M\right\}} J_{\mu}^{N_{e}}\left(\Phi_{\tau^{\prime}}^{\tau}\right) n_{\Sigma_{t^{*}}}^{\mu} \\
\quad+\int_{-\infty}^{\infty} d t^{*} \int_{\{r \geq R\}}\left(2 f\left(r^{2}+a^{2}\right)^{1 / 2}\left(F_{b}+F_{\mathrm{d}}+F_{\natural}+F_{\sharp}\right) \partial_{r^{*}}\left(\left(r^{2}+a^{2}\right)^{1 / 2} \Phi_{\tau^{\prime}}^{\tau}\right)\right. \\
\left.+f^{\prime}\left(r^{2}+a^{2}\right)\left(F_{b}+F_{\mathrm{d}}+F_{\sharp}+F_{\sharp}\right) \Phi_{\tau^{\prime}}^{\tau}\right) \frac{\Delta}{r^{2}+a^{2}} \sin \theta d \phi d \theta d r^{*} .
\end{gathered}
$$

By the definition of the cutoff, we have the pointwise equalities

$$
F=F_{b}+F_{\mathrm{d}}+F_{\natural}+F_{\sharp} .
$$


Therefore, we have

$$
\begin{aligned}
& \iint_{\mathscr{R}(-\infty, \infty)} \chi \mathbb{1}_{\left\{|r-3 M| \geq \frac{1}{8} M\right\}} J_{\mu}^{N_{e}}\left(\Phi_{\tau^{\prime}}^{\tau}\right) n_{\Sigma_{t^{*}}}^{\mu}+\chi\left(\Phi_{\tau^{\prime}}^{\tau}\right)^{2} \\
& \leq C \int_{\mathscr{H}\left(\tau^{\prime}-1, \tau+1\right)} J_{\mu}^{N_{e}}(\Phi) n_{\mathscr{H} \mathcal{C}^{+}}^{\mu}+C\left(\delta^{\prime}\right)^{-1} \iint_{\mathscr{R}(-\infty, \infty) \cap\{r \leq R\}} F^{2} \\
& \quad+C \delta^{\prime} \iint_{\mathscr{R}(-\infty, \infty) \cap\{r \leq R\}}\left(\Phi_{\tau^{\prime}}^{\tau}\right)^{2}+\left(\partial_{r^{*}} \Phi_{\tau^{\prime}}^{\tau}\right)^{2}+\mathbb{1}_{\left\{r \leq \frac{23}{8} M\right\}} J_{\mu}^{N_{e}}\left(\Phi_{\tau^{\prime}}^{\tau}\right) n_{\Sigma_{t^{*}}}^{\mu} \\
& \quad+\int_{-\infty}^{\infty} d t^{*} \int_{\{r \geq R\}}\left(2 f\left(r^{2}+a^{2}\right)^{1 / 2} F \partial_{r^{*}}\left(\left(r^{2}+a^{2}\right)^{1 / 2} \Phi_{\tau^{\prime}}^{\tau}\right)+f^{\prime}\left(r^{2}+a^{2}\right) F \Phi_{\tau^{\prime}}^{\tau}\right) \frac{\Delta}{r^{2}+a^{2}} \sin \theta d \phi d \theta d r^{*} .
\end{aligned}
$$

First, by Proposition 10, we have

$$
\int_{\mathscr{H}\left(\tau^{\prime}-1, \tau+1\right)} J_{\mu}^{N_{e}}(\Phi) n_{\mathscr{H} \mathcal{H}^{+}}^{\mu} \leq C \int_{\Sigma_{\tau^{\prime}}} J_{\mu}^{N_{e}}(\Phi) n_{\Sigma_{\tau^{\prime}}}^{\mu}+C \int_{\Sigma_{\tau}} J_{\mu}^{N_{e}}(\Phi) n_{\Sigma_{\tau}}^{\mu}+\int_{\mathscr{H}\left(\tau^{\prime}, \tau\right)} J_{\mu}^{N_{e}}(\Phi) n_{\mathscr{H} \mathcal{H}^{+}}^{\mu} .
$$

Recall that

$$
F=\xi G+2 D^{\alpha} \Phi D_{\alpha} \xi+\Phi \square_{g_{K}} \xi
$$

By the definition of $\xi$, the last two terms are supported in the $t^{*}$ range $\left(\tau^{\prime}-1, \tau^{\prime}\right) \cup(\tau, \tau+1)$. Moreover, since $\xi$ depends only on $t^{*}$, the only terms involving $D \Phi$ are $\partial_{t^{*}} \Phi$ and $O(\epsilon) \partial_{\phi^{*}} \Phi$. Using this, we immediately have the following with $C$ independent of $e$ as long as $\epsilon \ll e$ :

$$
\begin{aligned}
& C\left(\delta^{\prime}\right)^{-1} \iint_{\mathscr{R}^{(-\infty, \infty)} \cap\{r \leq R\}} F^{2} \\
& \leq C\left(\delta^{\prime}\right)^{-1}\left(\iint_{\mathscr{R}_{\left(\tau^{\prime}-1, \tau+1\right) \cap\{r \leq R\}}} G^{2}+\iint_{\mathscr{R}\left(\tau^{\prime}-1, \tau^{\prime}\right) \cup \mathscr{R}(\tau, \tau+1)}\left(r^{-2} \Phi^{2}+J_{\mu}^{N_{e}}(\Phi) n_{\Sigma_{t^{*}}}^{\mu}\right)\right) .
\end{aligned}
$$

Similarly, we have

$$
\begin{aligned}
\int_{-\infty}^{\infty} d t^{*} \int_{\{r \geq R\}} f^{\prime}\left(r^{2}+\right. & \left.a^{2}\right) F \Phi_{\tau^{\prime}}^{\tau} \frac{\Delta}{r^{2}+a^{2}} \sin \theta d \phi d \theta d r^{*} \\
& \leq C\left(\iint_{\mathscr{R}_{\left(\tau^{\prime}-1, \tau+1\right)}} r^{-1}|\Phi||G|+\iint_{\mathscr{R}_{\left(\tau^{\prime}-1, \tau^{\prime}\right) \cup \Re(\tau, \tau+1)}}\left(r^{-2} \Phi^{2}+J_{\mu}^{N_{e}}(\Phi) n_{\Sigma_{t^{*}}}^{\mu}\right)\right) .
\end{aligned}
$$

The other term with $F$ is more delicate to estimate. One of the terms in the expansion does not have sufficient decay in $r$ :

$$
\begin{aligned}
\int_{-\infty}^{\infty} d t^{*} \int_{\{r \geq R\}} 2 f\left(r^{2}+a^{2}\right)^{1 / 2} F \partial_{r^{*}}\left(\left(r^{2}+a^{2}\right)^{1 / 2} \Phi_{\tau^{\prime}}^{\tau}\right) \frac{\Delta}{r^{2}+a^{2}} \sin \theta d \phi d \theta d r^{*} \\
\leq C\left(\iint_{\mathscr{R}\left(\tau^{\prime}-1, \tau+1\right)} r^{-1}|\Phi||G|+\iint_{\mathscr{R}\left(\tau^{\prime}-1, \tau^{\prime}\right) \cup \mathscr{R}(\tau, \tau+1)}\left(r^{-2} \Phi^{2}+J_{\mu}^{N_{e}}(\Phi) n_{\Sigma_{t^{*}}}^{\mu}\right)\right) \\
\quad+\int_{-\infty}^{\infty} d t^{*} \int_{\{r \geq R\}} 2 f\left(r^{2}+a^{2}\right)^{1 / 2} \Phi \square_{g_{K}} \xi \partial_{r^{*}}\left(\left(r^{2}+a^{2}\right)^{1 / 2} \Phi\right) \xi \frac{\Delta}{r^{2}+a^{2}} \sin \theta d \phi d \theta d r^{*}
\end{aligned}
$$


Nevertheless, noting that $\xi$ is independent of $r^{*}$, an integration by parts in $r^{*}$ would give

$$
\begin{aligned}
\int_{-\infty}^{\infty} d t^{*} & \int_{\{r \geq R\}} 2 f\left(r^{2}+a^{2}\right)^{1 / 2} \Phi \square_{g_{K}} \xi \partial_{r^{*}}\left(\left(r^{2}+a^{2}\right)^{1 / 2} \Phi\right) \xi \frac{\Delta}{r^{2}+a^{2}} \sin \theta d \phi d \theta d r^{*} \\
& =-\int_{-\infty}^{\infty} d t^{*} \int_{\{r \geq R\}}\left(r^{2}+a^{2}\right) \Phi^{2} \xi \square_{g_{K}} \xi \partial_{r^{*}}\left(f \frac{\Delta}{r^{2}+a^{2}}\right) \sin \theta d \phi d \theta d r^{*}+\text { boundary terms } \\
& \leq C \iint_{\mathscr{R}\left(\tau^{\prime}-1, \tau^{\prime}\right) \cup \Re(\tau, \tau+1)} r^{-2} \Phi^{2},
\end{aligned}
$$

where the boundary terms can be controlled (after possibly changing $R$ ) by pigeonholing in $r \in[R, R+1]$. By the mild growth estimate of Proposition 10, the estimate near the event horizon from Proposition 18 and the Hardy inequality of Proposition 21,

$$
\begin{aligned}
\iint_{\mathscr{R}\left(\tau^{\prime}-1, \tau^{\prime}\right) \cup \Re(\tau, \tau+1)}\left(r^{-2} \Phi^{2}+J_{\mu}^{N_{e}}(\Phi) n_{\Sigma_{t^{*}}}^{\mu}\right) & \\
& \leq C\left(\int_{\Sigma_{\tau^{\prime}}} J_{\mu}^{N_{e}}(\Phi) n_{\Sigma_{\tau^{\prime}}}^{\mu}+\int_{\Sigma_{\tau}} J_{\mu}^{N_{e}}(\Phi) n_{\Sigma_{\tau^{\prime}}}^{\mu}+\iint_{\mathscr{R}\left(\tau^{\prime}-1, \tau^{\prime}\right) \cup \Re(\tau, \tau+1)} G^{2}\right) .
\end{aligned}
$$

Therefore, using all the estimates above and noticing the support of $\xi$, we have

$$
\begin{aligned}
& \iint_{\mathscr{R}\left(\tau^{\prime}, \tau\right)} \chi\left(\mathbb{1}_{\left\{|r-3 M| \geq \frac{1}{8} M\right\}} J_{\mu}^{N_{e}}(\Phi) n_{\Sigma_{t^{*}}}^{\mu}+\Phi^{2}\right) \\
& \leq C\left(\delta^{\prime}\right)^{-1} \int_{\Sigma_{\tau^{\prime}}} J_{\mu}^{N_{e}}(\Phi) n_{\Sigma_{\tau^{\prime}}}^{\mu}+C\left(\delta^{\prime}\right)^{-1} \int_{\Sigma_{\tau}} J_{\mu}^{N_{e}}(\Phi) n_{\Sigma_{\tau}}^{\mu}+C \int_{\mathscr{H}\left(\tau^{\prime}, \tau\right)} J_{\mu}^{N_{e}}(\Phi) n_{\mathscr{H}^{+}}^{\mu} \\
& +C \iint_{\mathscr{R}\left(\tau^{\prime}-1, \tau+1\right)} r^{-1}|\Phi||G|+C\left(\delta^{\prime}\right)^{-1} \iint_{\mathscr{R}\left(\tau^{\prime}-1, \tau+1\right)} G^{2} \\
& +C \delta^{\prime} \iint_{\mathscr{R}\left(\tau^{\prime}, \tau\right) \cap\{r \leq R\}} \Phi^{2}+\left(\partial_{r^{*}} \Phi\right)^{2}+\mathbb{1}_{\left\{r \leq \frac{23}{8} M\right\}} J_{\mu}^{N_{e}(\Phi) n_{\Sigma_{t^{*}}}^{\mu}}
\end{aligned}
$$

We add to this the estimates near spatial infinity and the event horizon, that is, Propositions 15 and 18 , to get

$$
\begin{aligned}
& \iint_{\mathscr{R}\left(\tau^{\prime}, \tau\right)} r^{-1-\delta_{1}} \mathbb{1}_{\left\{|r-3 M| \geq \frac{1}{8} M\right\}} J_{\mu}^{N_{e}}(\Phi) n_{\Sigma_{t^{*}}}^{\mu}+r^{-1-\delta}\left(\partial_{r} \Phi\right)^{2}+r^{-3-\delta} \Phi^{2} \\
& \leq C\left(\delta^{\prime}\right)^{-1} \int_{\Sigma_{\tau^{\prime}}} J_{\mu}^{N}(\Phi) n_{\Sigma_{\tau^{\prime}}}^{\mu}+C\left(\delta^{\prime}\right)^{-1} \int_{\Sigma_{\tau}} J_{\mu}^{N_{e}}(\Phi) n_{\Sigma_{\tau}}^{\mu}+C \int_{\mathscr{H}\left(\tau^{\prime}, \tau\right)} J_{\mu}^{N_{e}}(\Phi) n_{\mathscr{H}^{+}}^{\mu} \\
& \quad+C \iint_{\mathscr{R}\left(\tau^{\prime}-1, \tau+1\right)}\left(\left|\partial_{r^{*}} \Phi\right|+r^{-1}|\Phi|\right)|G|+C\left|\iint_{\mathscr{R}\left(\tau^{\prime}-1, \tau+1\right) \cap\left\{r \leq \frac{23}{8} M\right\}}\left(\partial_{t^{*}} \Phi+e Y \Phi\right) G\right| \\
& \quad+C\left(\delta^{\prime}\right)^{-1} \iint_{\mathscr{R}\left(\tau^{\prime}-1, \tau+1\right)} G^{2}+C \delta^{\prime} \iint_{\mathscr{R}\left(\tau^{\prime}-1, \tau+1\right) \cap\{r \leq R\}} \Phi^{2}+\left(\partial_{r^{*}} \Phi\right)^{2}+\mathbb{1}_{\left\{r \leq \frac{23}{8} M\right\}} J_{\mu}^{N}(\Phi) n_{\Sigma_{t^{*}}}^{\mu}
\end{aligned}
$$


By choosing $\delta^{\prime}$ sufficiently small and absorbing $\left(\delta^{\prime}\right)^{-1}$ into the constant $C$, we can absorb the last term:

$$
\begin{aligned}
& \iint_{\mathscr{R}\left(\tau^{\prime}, \tau\right)} r^{-1-\delta} \mathbb{1}_{\left\{|r-3 M| \geq \frac{1}{8} M\right\}} J_{\mu}^{N_{e}}(\Phi) n_{\Sigma_{t^{*}}}^{\mu}+r^{-1-\delta}\left(\partial_{r} \Phi\right)^{2}+r^{-3-\delta} \Phi^{2} \\
& \leq C \int_{\Sigma_{\tau^{\prime}}} J_{\mu}^{N_{e}}(\Phi) n_{\Sigma_{\tau^{\prime}}}^{\mu}+C \int_{\Sigma_{\tau}} J_{\mu}^{N_{e}}(\Phi) n_{\Sigma_{\tau}}^{\mu}+C \int_{\mathscr{H}\left(\tau^{\prime}, \tau\right)} J_{\mu}^{N_{e}}(\Phi) n_{\mathscr{H}^{+}}^{\mu} \\
& \quad+C \iint_{\mathscr{R}\left(\tau^{\prime}-1, \tau+1\right) \cap\left\{r \leq \frac{23}{8} M\right\}}\left(\left|\partial_{r^{*}} \Phi\right|+r^{-1}|\Phi|\right)|G|+C\left|\iint_{\mathscr{R}\left(\tau^{\prime}-1, \tau+1\right) \cap\left\{r \leq \frac{23}{8} M\right\}}\left(\partial_{t^{*}} \Phi+e Y \Phi\right) G\right| \\
& \quad+C \iint_{\mathscr{R}\left(\tau^{\prime}-1, \tau+1\right)} G^{2} .
\end{aligned}
$$

using Proposition 10 and 21 at the last step.

Definition 23. From now on, we write

$$
\begin{aligned}
& K^{X_{0}}(\Phi)=r^{-1-\delta} \mathbb{1}_{\left\{|r-3 M| \geq \frac{1}{8} M\right\}} J_{\mu}^{N}(\Phi) n_{\Sigma_{\tau}}^{\mu}+r^{-1-\delta}\left(\partial_{r} \Phi\right)^{2}+r^{-3-\delta} \Phi^{2}, \\
& K^{X_{1}}(\Phi)=r^{-1-\delta} J_{\mu}^{N}(\Phi) n_{\Sigma_{\tau}}^{\mu}+r^{-3-\delta} \Phi^{2}
\end{aligned}
$$

This is a slight abuse of notation because these "currents" do not arise directly from a vector field.

\subsection{Boundedness of the nondegenerate energy.}

Proposition 24. Let $\Phi$ satisfy $\square_{g_{K}} \Phi=G$. For e sufficiently small and $\epsilon \ll e$, we have

$$
\begin{aligned}
& \int_{\Sigma_{\tau}} J_{\mu}^{N_{e}}(\Phi) n_{\Sigma_{\tau}}^{\mu}+\int_{\mathscr{H}\left(\tau^{\prime}, \tau\right)} J_{\mu}^{N_{e}}(\Phi) n_{\mathscr{H}^{+}}^{\mu}+\iint_{\mathscr{R}\left(\tau^{\prime}, \tau\right) \cap\left\{r \leq r_{Y}^{-}\right\}} K_{\mu}^{N_{e}}(\Phi) \\
& \leq C\left(\int_{\Sigma_{\tau^{\prime}}} J_{\mu}^{N_{e}}(\Phi) n_{\Sigma_{\tau^{\prime}}}^{\mu}+\left|\iint_{\mathscr{R}\left(\tau^{\prime}-1, \tau+1\right)} \partial_{t^{*}} \Phi G\right|+\left|\iint_{\mathscr{R}\left(\tau^{\prime}-1, \tau+1\right)} e Y \Phi G\right|\right. \\
& \left.+\iint_{\mathscr{R}\left(\tau^{\prime}-1, \tau+1\right)}\left(\left|\partial_{r} \Phi\right|+r^{-1}|\Phi|\right)|G|+\iint_{\mathscr{R}\left(\tau^{\prime}-1, \tau+1\right)} G^{2}\right) .
\end{aligned}
$$

Proof. We recall that

$$
\iint_{\mathscr{R}\left(\tau^{\prime}, \tau\right) \cap\left\{r_{Y}^{-} \leq r \leq r_{Y}^{+}\right\}} K^{Y}(\Phi) \leq C \int_{\tau^{\prime}}^{\tau} \int_{\Sigma_{\bar{\tau}}} J_{\mu}^{N_{e}}(\Phi) n_{\Sigma_{t^{*}}}^{\mu} d t^{*},
$$

with $C$ independent of $e$ and $\epsilon$ whenever $\epsilon \ll e<1$. At this point, we choose $r_{Y}^{+}<\frac{11}{4} M<\frac{23}{8} M$. Hence this term can be controlled by the integrated decay estimates. Then, by Proposition 9 ,

$$
\begin{aligned}
& \int_{\Sigma_{\tau}} J_{\mu}^{N_{e}}(\Phi) n_{\Sigma_{\tau}}^{\mu}+\int_{\mathscr{H}\left(\tau^{\prime}, \tau\right)} J_{\mu}^{N_{e}}(\Phi) n_{\mathscr{H}^{+}}^{\mu}+\iint_{\mathscr{R}\left(\tau^{\prime}, \tau\right) \cap\left\{r \leq r_{Y}^{-}\right\}} K_{\mu}^{N_{e}}(\Phi) \\
& =\int_{\Sigma_{\tau^{\prime}}} J_{\mu}^{N_{e}}(\Phi) n_{\Sigma_{\tau^{\prime}}}^{\mu}+e \iint_{\mathscr{R}\left(\tau^{\prime}, \tau\right) \cap\left\{r_{Y}^{-} \leq r \leq r_{Y}^{+}\right\}} K^{Y}(\Phi)+\iint_{\mathscr{R}\left(\tau^{\prime}, \tau\right)}\left(\partial_{t^{*}} \Phi+e Y \Phi\right) G \\
& \leq \int_{\Sigma_{\tau^{\prime}}} J_{\mu}^{N_{e}}(\Phi) n_{\Sigma_{\tau^{\prime}}}^{\mu}+C e \int_{\tau^{\prime}}^{\tau} \int_{\Sigma_{\bar{\tau}} \cap\left\{r_{Y}^{-} \leq r \leq r_{Y}^{+}\right\}} J_{\mu}^{N_{e}}(\Phi) n_{\Sigma_{\bar{\tau}}}^{\mu} d \bar{\tau}+\left|\iint_{\mathscr{R}\left(\tau^{\prime}, \tau\right)}\left(\partial_{t^{*}} \Phi+e Y \Phi\right) G\right|
\end{aligned}
$$




$$
\begin{aligned}
\leq \int_{\Sigma_{\tau^{\prime}}} J_{\mu}^{N_{e}}(\Phi) n_{\Sigma_{\tau^{\prime}}}^{\mu}+C e\left(\int_{\Sigma_{\tau}} J_{\mu}^{N_{e}}(\Phi) n_{\Sigma_{\tau}}^{\mu}\right. & +\int_{\Sigma_{\tau^{\prime}}} J_{\mu}^{N_{e}}(\Phi) n_{\Sigma_{\tau^{\prime}}}^{\mu}+\int_{\mathscr{H}\left(\tau^{\prime}, \tau\right)} J_{\mu}^{N_{e}}(\Phi) n_{\mathscr{H}}^{\mu} \\
& \left.+\iint_{\mathscr{R}\left(\tau^{\prime}-1, \tau+1\right)}\left(\left|\partial_{r} \Phi\right|+r^{-1}|\Phi|\right)|G|+\iint_{\mathscr{R}\left(\tau^{\prime}-1, \tau+1\right)} G^{2}\right) \\
& +\left|\iint_{\mathscr{R}\left(\tau^{\prime}, \tau\right)}\left(\partial_{t^{*}} \Phi+e Y \Phi\right) G\right| .
\end{aligned}
$$

Hence, the proposition holds if $e$ is chosen to be sufficiently small.

Remark. From this point on, we will consider $r_{Y}^{+}$and $e$ to be fixed. After $e$ is fixed, the vector field $N_{e}$ will be written simply as $N$.

We now estimate the inhomogeneous terms in Proposition 24:

\section{Proposition 25.}

$$
\begin{aligned}
\int_{\Sigma_{\tau}} J_{\mu}^{N}(\Phi) n_{\Sigma_{\tau}}^{\mu}+\int_{\mathscr{H}\left(\tau^{\prime}, \tau\right)} J_{\mu}^{N}(\Phi) & n_{\mathscr{H} \mathcal{H}^{+}}^{\mu}+\iint_{\mathscr{R}\left(\tau^{\prime}, \tau\right) \cap\left\{r \leq r_{Y}^{-}\right\}} K^{N}(\Phi)+\iint_{\mathscr{R}\left(\tau^{\prime}, \tau\right)} K^{X_{0}}(\Phi) \\
& \leq C\left(\int_{\Sigma_{\tau^{\prime}}} J_{\mu}^{N}(\Phi) n_{\Sigma_{\tau^{\prime}}}^{\mu}+\left(\int_{\tau^{\prime}-1}^{\tau+1}\left(\int_{\Sigma_{t^{*}}} G^{2}\right)^{1 / 2} d t^{*}\right)^{2}+\iint_{\mathscr{R}\left(\tau^{\prime}, \tau\right)} G^{2}\right) .
\end{aligned}
$$

Proof. Adding the estimates in Propositions 22 and $\delta$ times the estimates in Proposition 24, we have

$$
\begin{array}{r}
\int_{\Sigma_{\tau}} J_{\mu}^{N}(\Phi) n_{\Sigma_{\tau}}^{\mu}+\int_{\mathscr{H}\left(\tau^{\prime}, \tau\right)} J_{\mu}^{N}(\Phi) n_{\mathscr{H} \mathcal{H}^{+}}^{\mu}+\iint_{\mathscr{R}\left(\tau^{\prime}, \tau\right) \cap\left\{r \leq r_{Y}^{-}\right\}} K^{N}(\Phi)+\delta \iint_{\mathscr{R}\left(\tau^{\prime}, \tau\right)} K^{X_{0}}(\Phi) \\
\leq C\left(\int_{\Sigma_{\tau^{\prime}}} J_{\mu}^{N}(\Phi) n_{\Sigma_{\tau^{\prime}}}^{\mu}+\iint_{\mathscr{R}\left(\tau^{\prime}-1, \tau+1\right)}\left(|\partial \Phi|+r^{-1}|\Phi|\right)|G|+\iint_{\mathscr{R}\left(\tau^{\prime}-1, \tau+1\right)} G^{2}\right) \\
+C \delta\left(\int_{\Sigma_{\tau}} J_{\mu}^{N}(\Phi) n_{\Sigma_{\tau}}^{\mu}+\int_{\mathscr{H}\left(\tau^{\prime}, \tau\right)} J_{\mu}^{N}(\Phi) n_{\mathscr{H} \mathcal{H}^{+}}^{\mu}\right) \\
\leq C\left(\int_{\Sigma_{\tau^{\prime}}} J_{\mu}^{N}(\Phi) n_{\Sigma_{\tau^{\prime}}}^{\mu}+\sup _{t^{*} \in\left[\tau^{\prime}-1, \tau+1\right]}\left(\int_{\Sigma_{t^{*}}} J_{\mu}^{N}(\Phi) n_{\Sigma_{t^{*}}}^{\mu}\right)^{1 / 2} \int_{\tau^{\prime}-1}^{\tau+1}\left(\int_{\Sigma_{t^{*}}} G^{2}\right)^{1 / 2} d t^{*}\right. \\
\left.\quad+\iint_{\mathscr{R}\left(\tau^{\prime}-1, \tau+1\right)} G^{2}\right)+C \delta\left(\int_{\Sigma_{\tau}} J_{\mu}^{N}(\Phi) n_{\Sigma_{\tau}}^{\mu}+\int_{\mathscr{H}\left(\tau^{\prime}, \tau\right)} J_{\mu}^{N}(\Phi) n_{\mathscr{H}}^{\mu}\right),
\end{array}
$$

where at the last step we have used Proposition 21. Choosing $C \delta \leq \frac{1}{2}$, we can absorb the last term to the left side to get

$$
\begin{aligned}
\int_{\Sigma_{\tau}} J_{\mu}^{N}(\Phi) n_{\Sigma_{\tau}}^{\mu}+\int_{\mathscr{H}\left(\tau^{\prime}, \tau\right)} J_{\mu}^{N}(\Phi) n_{\mathscr{H} \mathcal{H}^{+}}^{\mu}+\iint_{\mathscr{R}\left(\tau^{\prime}, \tau\right) \cap\left\{r \leq r_{Y}^{-}\right\}} K^{N}(\Phi)+\delta \iint_{\mathscr{R}\left(\tau^{\prime}, \tau\right)} K^{X_{0}}(\Phi) \\
\leq C\left(\int_{\Sigma_{\tau^{\prime}}} J_{\mu}^{N}(\Phi) n_{\Sigma_{\tau^{\prime}}}^{\mu}+\sup _{t^{*} \in\left[\tau^{\prime}-1, \tau+1\right]}\left(\int_{\Sigma_{t^{*}}} J_{\mu}^{N}(\Phi) n_{\Sigma_{t^{*}}}^{\mu}\right)^{1 / 2} \int_{\tau^{\prime}-1}^{\tau+1}\left(\int_{\Sigma_{t^{*}}} G^{2}\right)^{1 / 2} d t^{*}\right. \\
\left.+\iint_{\mathscr{R}\left(\tau^{\prime}-1, \tau+1\right)} G^{2}\right) .
\end{aligned}
$$


By considering the estimate above on $\left[\tau^{\prime}, \tilde{\tau}\right]$, where $\tilde{\tau}$ is when the supremum on the right-hand side is achieved, and using Proposition 10, we get

$$
\begin{aligned}
\int_{\Sigma_{\tilde{\tau}}} J_{\mu}^{N}(\Phi) n_{\Sigma_{\tilde{\tau}}}^{\mu}+\int_{\mathscr{H}\left(\tau^{\prime}, \tilde{\tau}\right)} J_{\mu}^{N}(\Phi) n_{\mathscr{H}+}^{\mu}+\iint_{\mathscr{R}\left(\tau^{\prime}, \tilde{\tau}\right) \cap\left\{r \leq r_{Y}^{-}\right\}} K^{N}(\Phi)+\delta \iint_{\mathscr{R}\left(\tau^{\prime}, \tilde{\tau}\right)} K^{X_{0}}(\Phi) \\
\leq C\left(\int_{\Sigma_{\tau^{\prime}}} J_{\mu}^{N}(\Phi) n_{\Sigma_{\tau^{\prime}}}^{\mu}+\left(\int_{\tau^{\prime}-1}^{\tau+1}\left(\int_{\Sigma_{t^{*}}} G^{2}\right)^{1 / 2} d t^{*}\right)^{2}\right) .
\end{aligned}
$$

We plug this into (7) and apply Cauchy-Schwarz to prove the proposition.

We can also estimate the inhomogeneous terms not in $L^{1} L^{2}$ but in $L^{2} L^{2}$, provided that we allow some extra factors of $r$ and some loss of derivatives in $G$. This is especially useful for estimating the commutator terms from $S$, which do not have sufficient decay in $t^{*}$ in the interior to be estimated in $L^{1} L^{2}$. More precisely:

Proposition 26. $\int_{\Sigma_{\tau}} J_{\mu}^{N}(\Phi) n_{\Sigma_{\tau}}^{\mu}+\int_{\mathscr{H}\left(\tau^{\prime}, \tau\right)} J_{\mu}^{N}(\Phi) n_{\mathscr{H}}^{\mu}+\iint_{\mathscr{R}\left(\tau^{\prime}, \tau\right) \cap\left\{r \leq r_{Y}^{-}\right\}} K^{N}(\Phi)+\iint_{\mathscr{R}^{+}\left(\tau^{\prime}, \tau\right)} K^{X_{0}}(\Phi)$

$$
\leq C\left(\int_{\Sigma_{\tau^{\prime}}} J_{\mu}^{N}(\Phi) n_{\Sigma_{\tau^{\prime}}}^{\mu}+\sum_{m=0}^{1} \iint_{\mathscr{R}\left(\tau^{\prime}-1, \tau+1\right)} r^{1+\delta}\left(\partial_{t^{*}}^{m} G\right)^{2}+\sup _{t^{*} \in\left[\tau^{\prime}-1, \tau+1\right]} \int_{\Sigma_{t^{*}} \cap\left\{|r-3 M| \leq \frac{1}{8} M\right\}} G^{2}\right) .
$$

Proof. By Propositions 22 and 24,

$$
\begin{aligned}
& \int_{\Sigma_{\tau}} J_{\mu}^{N}(\Phi) n_{\Sigma_{\tau}}^{\mu}+\int_{\mathscr{H}\left(\tau^{\prime}, \tau\right)} J_{\mu}^{N}(\Phi) n_{\mathscr{H}+^{+}}^{\mu}+\iint_{\mathscr{R}\left(\tau^{\prime}, \tau\right) \cap\left\{r \leq r_{Y}^{-}\right\}} K^{N}(\Phi)+\delta \iint_{\mathscr{R}\left(\tau^{\prime}, \tau\right)} K^{X_{0}}(\Phi) \\
& \leq C\left(\int_{\Sigma_{\tau^{\prime}}} J_{\mu}^{N_{e}(\Phi)} n_{\Sigma_{\tau^{\prime}}}^{\mu}+\left|\iint_{\mathscr{R}\left(\tau^{\prime}-1, \tau+1\right)} \partial_{t^{*}} \Phi G\right|\right. \\
& \left.+\left|\iint_{\mathscr{R}\left(\tau^{\prime}-1, \tau+1\right)} e Y \Phi G\right|+\iint_{\mathscr{R}\left(\tau^{\prime}-1, \tau+1\right)}\left(\left|\partial_{r} \Phi\right|+r^{-1}|\Phi|\right)|G|+\iint_{\mathscr{R}\left(\tau^{\prime}-1, \tau+1\right)} G^{2}\right) \\
& \quad+C \delta^{\prime}\left(\int_{\Sigma_{\tau}} J_{\mu}^{N}(\Phi) n_{\Sigma_{\tau}}^{\mu}+\int_{\mathscr{H}\left(\tau^{\prime}, \tau\right)} J_{\mu}^{N}(\Phi) n_{\mathscr{H} \mathcal{H}^{+}}^{\mu}\right) .
\end{aligned}
$$

Choosing $C \delta^{\prime} \leq \frac{1}{2}$, we can absorb the last term into the left hand side to get

$$
\begin{aligned}
& \int_{\Sigma_{\tau}} J_{\mu}^{N}(\Phi) n_{\Sigma_{\tau}}^{\mu}+ \int_{\mathscr{H}\left(\tau^{\prime}, \tau\right)} J_{\mu}^{N}(\Phi) n_{\mathscr{H}+^{+}}^{\mu}+\iint_{\mathscr{R}\left(\tau^{\prime}, \tau\right) \cap\left\{r \leq r_{Y}^{-}\right\}} K^{N}(\Phi)+\iint_{\mathscr{R}\left(\tau^{\prime}, \tau\right)} K^{X_{0}}(\Phi) \\
& \leq C\left(\int_{\Sigma_{\tau^{\prime}}} J_{\mu}^{N}(\Phi) n_{\Sigma_{\tau^{\prime}}}^{\mu}+\left|\iint_{\mathscr{R}\left(\tau^{\prime}-1, \tau+1\right)} \partial_{t^{*}} \Phi G\right|+\left|\iint_{\mathscr{R}\left(\tau^{\prime}-1, \tau+1\right)} e Y \Phi G\right|\right. \\
&\left.\quad+\iint_{\mathscr{R}\left(\tau^{\prime}-1, \tau+1\right)}\left(\left|\partial_{r} \Phi\right|+r^{-1}|\Phi|\right)|G|+\iint_{\mathscr{R}\left(\tau^{\prime}-1, \tau+1\right)} G^{2}\right) .
\end{aligned}
$$


For the bulk error term, we focus at the region $\left\{|r-3 M| \leq \frac{1}{8} M\right\}$ and integrate by parts.

$$
\begin{aligned}
& \left|\iint_{\mathscr{R}\left(\tau^{\prime}-1, \tau+1\right) \cap\left\{|r-3 M| \leq \frac{1}{8} M\right\}} \partial_{t^{*}} \Phi G\right| \\
& \leq \delta^{\prime} \iint_{\mathscr{R}\left(\tau^{\prime}-1, \tau+1\right) \cap\left\{|r-3 M| \leq \frac{1}{8} M\right\}} \Phi^{2}+C\left(\delta^{\prime}\right)^{-1} \iint_{\mathscr{R}\left(\tau^{\prime}-1, \tau+1\right) \cap\left\{|r-3 M| \leq \frac{1}{8} M\right\}}\left(\partial_{t^{*}} G\right)^{2} \\
& +\left|\int_{\Sigma_{\tau+1} \cap\left\{|r-3 M| \leq \frac{1}{8} M\right\}} \Phi G\right|+\left|\int_{\Sigma_{\tau^{\prime}-1} \cap\left\{|r-3 M| \leq \frac{1}{8} M\right\}} \Phi G\right| \\
& \leq \delta^{\prime} \iint_{\mathscr{R}\left(\tau^{\prime}-1, \tau+1\right) \cap\left\{|r-3 M| \leq \frac{1}{8} M\right\}} r^{-3-\delta} \Phi^{2}+C\left(\delta^{\prime}\right)^{-1} \iint_{\mathscr{R}\left(\tau^{\prime}-1, \tau+1\right) \cap\left\{|r-3 M| \leq \frac{1}{8} M\right\}}\left(\partial_{t^{*}} G\right)^{2} \\
& +\sup _{t^{*} \in\left[\tau^{\prime}-1, \tau+1\right]}\left(\delta \int_{\Sigma_{t^{*}} \cap\left\{|r-3 M| \leq \frac{1}{8} M\right\}} r^{-2} \Phi^{2}+C\left(\delta^{\prime}\right)^{-1} \int_{\Sigma_{t^{*} \cap\left\{|r-3 M| \leq \frac{1}{8} M\right\}}} G^{2}\right) \\
& \leq \delta^{\prime} \iint_{\mathscr{R}\left(\tau^{\prime}-1, \tau+1\right) \cap\left\{|r-3 M| \leq \frac{1}{8} M\right\}} r^{-3-\delta} \Phi^{2}+C\left(\delta^{\prime}\right)^{-1} \iint_{\mathscr{R}\left(\tau^{\prime}-1, \tau+1\right) \cap\left\{|r-3 M| \leq \frac{1}{8} M\right\}}\left(\partial_{t^{*}} G\right)^{2} \\
& +\sup _{t^{*} \in\left[\tau^{\prime}-1, \tau+1\right]}\left(\delta^{\prime} \int_{\Sigma_{t^{*}}} J_{\mu}^{N}(\Phi) n_{\Sigma_{t^{*}}}^{\mu}+C\left(\delta^{\prime}\right)^{-1} \int_{\Sigma_{t^{*} \cap\left\{|r-3 M| \leq \frac{1}{8} M\right\}}} G^{2}\right),
\end{aligned}
$$

where at the last step we used Proposition 21. Therefore,

$$
\begin{aligned}
& \int_{\Sigma_{\tau}} J_{\mu}^{N}(\Phi) n_{\Sigma_{\tau}}^{\mu}+\int_{\mathscr{H}\left(\tau^{\prime}, \tau\right)} J_{\mu}^{N}(\Phi) n_{\mathscr{H}^{+}}^{\mu}+\iint_{\mathscr{R}\left(\tau^{\prime}, \tau\right) \cap\left\{r \leq r_{Y}^{-}\right\}} K^{N}(\Phi)+\iint_{\mathscr{R}\left(\tau^{\prime}, \tau\right)} K^{X_{0}}(\Phi) \\
& \leq C\left(\int_{\Sigma_{\tau^{\prime}}} J_{\mu}^{N}(\Phi) n_{\Sigma_{\tau^{\prime}}}^{\mu}+\left|\iint_{\mathscr{R}\left(\tau^{\prime}-1, \tau+1\right)} \partial_{t^{*}} \Phi G\right|+\left|\iint_{\mathscr{R}\left(\tau^{\prime}-1, \tau+1\right)} e Y \Phi G\right|\right. \\
& \left.+\iint_{\mathscr{R}\left(\tau^{\prime}-1, \tau+1\right)}\left(\left|\partial_{r} \Phi\right|+r^{-1}|\Phi|\right)|G|+\iint_{\mathscr{R}\left(\tau^{\prime}-1, \tau+1\right)} G^{2}\right) \\
& \leq C\left(\int_{\Sigma_{\tau^{\prime}}} J_{\mu}^{N}(\Phi) n_{\Sigma_{\tau^{\prime}}}^{\mu}+\left|\iint_{\mathscr{R}\left(\tau^{\prime}-1, \tau+1\right) \cap\left\{|r-3 M| \leq \frac{1}{8} M\right\}} \partial_{t^{*}} \Phi G\right|\right)+C\left(\delta^{\prime}\right)^{-1} \iint_{\mathscr{R}\left(\tau^{\prime}-1, \tau+1\right)} r^{1+\delta} G^{2} \\
& +\delta^{\prime} \iint_{\mathscr{R}\left(\tau^{\prime}-1, \tau+1\right)}\left(r^{-3-\delta} \Phi^{2}+r^{-1-\delta}\left(\partial_{r} \Phi\right)^{2}+\mathbb{1}_{\left\{r \leq r_{Y}^{+}\right\}} J_{\mu}^{N}(\Phi) n_{\Sigma_{t^{*}}}^{\mu}\right) \\
& \leq C \int_{\Sigma_{\tau^{\prime}}} J_{\mu}^{N}(\Phi) n_{\Sigma_{\tau^{\prime}}}^{\mu}+C\left(\delta^{\prime}\right)^{-1} \sum_{m=0}^{1} \int_{\mathscr{R}\left(\tau^{\prime}-1, \tau+1\right)} r^{1+\delta}\left(\partial_{t^{*}}^{m} G\right)^{2}+\delta^{\prime} \iint_{\mathscr{R}\left(\tau^{\prime}-1, \tau+1\right)} K^{X_{0}}(\Phi) \\
& +\sup _{t^{*} \in\left[\tau^{\prime}-1, \tau+1\right]}\left(\delta^{\prime} \int_{\Sigma_{t^{*}}} J_{\mu}^{N}(\Phi) n_{\Sigma_{t^{*}}}^{\mu}+C\left(\delta^{\prime}\right)^{-1} \int_{\Sigma_{t^{*}} \cap\left\{|r-3 M| \leq \frac{1}{8} M\right\}} G^{2}\right) .
\end{aligned}
$$


where at the last step we have used Propositions 10 and 21. Suppose $\sup _{t^{*} \in\left[\tau^{\prime}-1, \tau+1\right]} \delta^{\prime} \int_{\Sigma_{t^{*}}} J_{\mu}^{N}(\Phi) n_{\Sigma_{t^{*}}}^{\mu}$ is achieved by $t^{*}=\tilde{\tau}$. Applying (8) on $\left[\tau^{\prime}, \tilde{\tau}\right]$, we get

$$
\begin{aligned}
\int_{\Sigma_{\tilde{\tau}}} J_{\mu}^{N}(\Phi) n_{\Sigma_{\tilde{\tau}}}^{\mu} \leq C \int_{\Sigma_{\tau^{\prime}}} J_{\mu}^{N}(\Phi) n_{\Sigma_{\tau^{\prime}}}^{\mu}+C\left(\delta^{\prime}\right)^{-1} & \sum_{m=0}^{1} \iint_{\mathscr{R}_{\left(\tau^{\prime}-1, \tau+1\right)}} r^{1+\delta}\left(\partial_{t^{*}}^{m} G\right)^{2}+\delta^{\prime} \iint_{\mathscr{R}\left(\tau^{\prime}-1, \tau+1\right)} K^{X_{0}}(\Phi) \\
& +\delta^{\prime} \int_{\Sigma_{\tilde{\tau}}} J_{\mu}^{N}(\Phi) n_{\Sigma_{\tilde{\tau}}}^{\mu}+C \sup _{t^{*} \in\left[\tau^{\prime}-1, \tau+1\right]} \int_{\Sigma_{t^{*} * \cap\left\{|r-3 M| \leq \frac{1}{8} M\right\}} G^{2},}
\end{aligned}
$$

which, upon choosing $\delta^{\prime} \leq \frac{1}{2}$ and subtracting the small term on both sides, gives

$$
\begin{array}{r}
\int_{\Sigma_{\tilde{\tau}}} J_{\mu}^{N}(\Phi) n_{\Sigma_{\tilde{\tau}}}^{\mu} \leq C \int_{\Sigma_{\tau^{\prime}}} J_{\mu}^{N}(\Phi) n_{\Sigma_{\tau^{\prime}}}^{\mu}+C\left(\delta^{\prime}\right)^{-1} \sum_{m=0}^{1} \iint_{\mathscr{R}\left(\tau^{\prime}-1, \tau+1\right)} r^{1+\delta}\left(\partial_{t^{*}}^{m} G\right)^{2}+\delta^{\prime} \iint_{\mathscr{R}\left(\tau^{\prime}-1, \tau+1\right)} K^{X_{0}}(\Phi) \\
+C \sup _{t^{*} \in\left[\tau^{\prime}-1, \tau+1\right]} \int_{\Sigma_{t^{*}} \cap\left\{|r-3 M| \leq \frac{1}{8} M\right\}} G^{2},
\end{array}
$$

Therefore, plugging this back into (8), we have

$$
\begin{aligned}
& \int_{\Sigma_{\tau}} J_{\mu}^{N}(\Phi) n_{\Sigma_{\tau}}^{\mu}+\int_{\mathscr{H}\left(\tau^{\prime}, \tau\right)} J_{\mu}^{N}(\Phi) n_{\mathscr{H} \mathcal{H}^{+}}^{\mu}+\iint_{\mathscr{R}\left(\tau^{\prime}, \tau\right) \cap\left\{r \leq r_{Y}^{-}\right\}} K^{N}(\Phi)+\iint_{\mathscr{R}\left(\tau^{\prime}, \tau\right)} K^{X_{0}}(\Phi) \\
& \leq C \int_{\Sigma_{\tau^{\prime}}} J_{\mu}^{N}(\Phi) n_{\Sigma_{\tau^{\prime}}}^{\mu}+\delta^{\prime} \iint_{\mathscr{R}\left(\tau^{\prime}, \tau\right)} K^{X_{0}}(\Phi)+\delta^{\prime} \iint_{\mathscr{R}\left(\tau^{\prime}-1, \tau^{\prime}\right) \cup \mathscr{R}(\tau, \tau+1)} K^{X_{0}}(\Phi) \\
& +C \sum_{m=0}^{1} \iint_{\mathscr{R}\left(\tau^{\prime}-1, \tau+1\right)} r^{1+\delta}\left(\partial_{t^{*}}^{m} G\right)^{2}+C \sup _{t^{*} \in\left[\tau^{\prime}-1, \tau+1\right]} \int_{\Sigma_{t^{*}} \cap\left\{|r-3 M| \leq \frac{1}{8} M\right\}} G^{2} \\
& \leq C \int_{\Sigma_{\tau^{\prime}}} J_{\mu}^{N}(\Phi) n_{\Sigma_{\tau^{\prime}}}^{\mu}+\delta^{\prime} \iint_{\mathscr{R}\left(\tau^{\prime}, \tau\right)} K^{X_{0}}(\Phi)+C \delta^{\prime} \int_{\Sigma_{\tau}} J_{\mu}^{N}(\Phi) n_{\Sigma_{\tau}}^{\mu} \\
& +C \sum_{m=0}^{1} \iint_{\mathscr{R}\left(\tau^{\prime}-1, \tau+1\right)} r^{1+\delta}\left(\partial_{t^{*}}^{m} G\right)^{2}+C \sup _{t^{*} \in\left[\tau^{\prime}-1, \tau+1\right]} \int_{\Sigma_{t^{*}} \cap\left\{|r-3 M| \leq \frac{1}{8} M\right\}} G^{2},
\end{aligned}
$$

where at the last step we have used Proposition 10. Finally, by choosing $C \delta \leq \frac{1}{2}$, we can absorb the small terms into the left-hand side and achieve the conclusion of the proposition.

In the proof of Proposition 26, there is a loss in derivative for $G$ because we have to integrate by parts in the region $\left\{|r-3 M| \leq \frac{1}{8} M\right\}$. Therefore, if $G$ is supported away from this region, we can repeat the proof without this loss. In other words:

Proposition 27. Suppose $G$ is supported away from $\left\{|r-3 M| \leq \frac{1}{8} M\right\}$. Then

$$
\begin{aligned}
\int_{\Sigma_{\tau}} J_{\mu}^{N}(\Phi) n_{\Sigma_{\tau}}^{\mu} & +\int_{\mathscr{H}\left(\tau^{\prime}, \tau\right)} J_{\mu}^{N}(\Phi) n_{\mathscr{H}^{+}}^{\mu}+\iint_{\mathscr{R}\left(\tau^{\prime}, \tau\right) \cap\left\{r \leq r_{Y}^{-}\right\}} K^{N}(\Phi)+\iint_{\mathscr{R}\left(\tau^{\prime}, \tau\right)} K^{X_{0}}(\Phi) \\
& \leq C\left(\int_{\Sigma_{\tau^{\prime}}} J_{\mu}^{N}(\Phi) n_{\Sigma_{\tau^{\prime}}}^{\mu}+\sum_{m=0}^{1} \iint_{\mathscr{R}^{\prime}\left(\tau^{\prime}-1, \tau+1\right)} r^{1+\delta} G^{2}+\sup _{t^{*} \in\left[\tau^{\prime}-1, \tau+1\right]} \int_{\Sigma_{t^{*}} \cap\left\{|r-3 M| \leq \frac{1}{8} M\right\}} G^{2}\right) .
\end{aligned}
$$


This will be useful in Section 13 .

In applications, it is useful to have both ways of estimating $G$.

Proposition 28. Let $G=G_{1}+G_{2}$ be any way to decompose the function $G$. Then

$$
\begin{aligned}
\int_{\Sigma_{\tau}} J_{\mu}^{N}(\Phi) n_{\Sigma_{\tau}}^{\mu} & +\int_{\mathscr{H}\left(\tau^{\prime}, \tau\right)} J_{\mu}^{N}(\Phi) n_{\mathscr{H}^{+}}^{\mu}+\iint_{\mathscr{R}\left(\tau^{\prime}, \tau\right) \cap\left\{r \leq r_{Y}^{-}\right\}} K^{N}(\Phi)+\iint_{\mathscr{R}\left(\tau^{\prime}, \tau\right)} K^{X_{0}}(\Phi) \\
& \leq C\left(\int_{\Sigma_{\tau^{\prime}}} J_{\mu}^{N}(\Phi) n_{\Sigma_{\tau^{\prime}}}^{\mu}+\left(\int_{\tau^{\prime}-1}^{\tau+1}\left(\int_{\Sigma_{t^{*}}} G_{1}^{2}\right)^{1 / 2} d t^{*}\right)^{2}+\iint_{\mathscr{R}\left(\tau^{\prime}-1, \tau+1\right)} G_{1}^{2}\right. \\
& +\sum_{m=0}^{1} \iint_{\mathscr{R}\left(\tau^{\prime}-1, \tau+1\right)} r^{1+\delta}\left(\partial_{t^{*}}^{m} G_{2}\right)^{2}+\sup _{t^{*} \in\left[\tau^{\prime}-1, \tau+1\right]} \int_{\left.\Sigma_{t^{*} \cap\left\{|r-3 M| \leq \frac{1}{8} M\right\}} G_{2}^{2}\right) .}
\end{aligned}
$$

In the estimates above, only the function $\Phi$ and its $\partial_{r}$ derivative can be estimated without a loss around the trapped set. To estimate the other derivatives, we need to commute with the Killing vector field $\partial_{t^{*}}$.

Proposition 29. We have

$$
\begin{aligned}
& \iint_{\mathscr{R}\left(\tau^{\prime}, \tau\right)} K^{X_{1}}(\Phi) \\
& \leq C\left(\sum_{m=0}^{1} \int_{\Sigma_{\tau^{\prime}}} J_{\mu}^{N}\left(\partial_{t^{*}}^{m} \Phi\right) n_{\Sigma_{\tau^{\prime}}}^{\mu}+\sum_{m=0}^{1}\left(\int_{\tau^{\prime}-1}^{\tau+1}\left(\int_{\Sigma_{t^{*}}}\left(\partial_{t^{*}}^{m} G_{1}\right)^{2}\right)^{1 / 2} d t^{*}\right)^{2}+\sum_{m=0}^{1} \iint_{\mathscr{R}\left(\tau^{\prime}-1, \tau+1\right)}\left(\partial_{t^{*}}^{m} G_{1}\right)^{2}\right. \\
& \quad+\sum_{m=0}^{2} \iint_{\mathscr{R}\left(\tau^{\prime}-1, \tau+1\right)} r^{1+\delta}\left(\partial_{t^{*}}^{m} G_{2}\right)^{2}+\sup _{t^{*} \in\left[\tau^{\prime}-1, \tau+1\right]} \sum_{m=0}^{1} \int_{\left.\Sigma_{t^{*} \cap\left\{|r-3 M| \leq \frac{1}{8} M\right\}}\left(\partial_{t^{*}}^{m} G_{2}\right)^{2}\right) .}
\end{aligned}
$$

Proof. Using Proposition 28 and the fact that $\partial_{t^{*}}$ is Killing, we immediately have the following estimate for $\partial_{t^{*}} \Phi$ :

$$
\begin{aligned}
& \iint_{\mathscr{R}\left(\tau^{\prime}, \tau\right)} r^{-3-\delta}\left(\partial_{t^{*}} \Phi\right)^{2} \\
& \leq C\left(\int_{\Sigma_{\tau^{\prime}}} J_{\mu}^{N}\left(\partial_{t^{*}} \Phi\right) n_{\Sigma_{\tau^{\prime}}}^{\mu}+\left(\int_{\tau^{\prime}-1}^{\tau+1}\left(\int_{\Sigma_{t^{*}}}\left(\partial_{t^{*}} G_{1}\right)^{2}\right)^{1 / 2} d t^{*}\right)^{2}+\iint_{\mathscr{R}\left(\tau^{\prime}-1, \tau+1\right)}\left(\partial_{t^{*}} G_{1}\right)^{2}\right. \\
& \left.+\sum_{m=1}^{2} \iint_{\mathscr{R}\left(\tau^{\prime}-1, \tau+1\right)} r^{1+\delta}\left(\partial_{t^{*}}^{m} G_{2}\right)^{2}+\sup _{t^{*} \in\left[\tau^{\prime}-1, \tau+1\right]} \int_{\Sigma_{t^{*}} \cap\left\{|r-3 M| \leq \frac{1}{8} M\right\}}\left(\partial_{t^{*}} G_{2}\right)^{2}\right) .
\end{aligned}
$$

This would allow us to estimate all derivatives of $\Phi$ except for the fact that the estimates for the angular derivatives of $\Phi$ degenerate around $r=3 M$ : 


$$
\begin{gathered}
\iint_{\mathscr{R}\left(\tau^{\prime}, \tau\right)}\left(\left.r^{-1-\delta} \mathbb{1}_{\left\{|r-3 M| \geq \frac{1}{8} M\right\}}|\not| \Phi\right|^{2}+r^{-1-\delta}\left(\partial_{r} \Phi\right)^{2}+r^{-1-\delta}\left(\partial_{t^{*}} \Phi\right)^{2}+r^{-3-\delta} \Phi^{2}\right) \\
\leq C\left(\sum_{m=0}^{1} \int_{\Sigma_{\tau^{\prime}}} J_{\mu}^{N}\left(\partial_{t^{*}}^{m} \Phi\right) n_{\Sigma_{\tau^{\prime}}}^{\mu}+\sum_{m=0}^{1}\left(\int_{\tau^{\prime}-1}^{\tau+1}\left(\int_{\Sigma_{t^{*}}}\left(\partial_{t^{*}}^{m} G_{1}\right)^{2}\right)^{1 / 2} d t^{*}\right)^{2}+\sum_{m=0}^{1} \iint_{\mathscr{R}\left(\tau^{\prime}-1, \tau+1\right)}\left(\partial_{t^{*}}^{m} G_{1}\right)^{2}\right. \\
\left.+\sum_{m=0}^{2} \iint_{\mathscr{R}\left(\tau^{\prime}-1, \tau+1\right)} r^{1+\delta}\left(\partial_{t^{*}}^{m} G_{2}\right)^{2}+\sup _{t^{*} \in\left[\tau^{\prime}-1, \tau+1\right]} \sum_{m=0}^{1} \int_{\Sigma_{t^{*}} \cap\left\{|r-3 M| \leq \frac{1}{8} M\right\}}\left(\partial_{t^{*}}^{m} G_{2}\right)^{2}\right) .
\end{gathered}
$$

We now use this known estimate and construct another vector field to control the angular derivatives in the region $r \sim 3 M$. The argument is simple because the estimate is only local. Take $f_{a n}(r)$ to be compactly support in $3 M-\frac{1}{4} M \leq r \leq 3 M+\frac{1}{4} M$ and identically equal to -1 in $3 M-\frac{1}{8} M \leq r \leq 3 M+\frac{1}{8} M$. If we consider $X_{a n}=f_{a n}(r) \partial_{r^{*}}$ in Schwarzschild spacetime, we get that the coefficient in front of the terms with angular derivatives is $\mu / 2 r$, which is bounded below in $3 M-\frac{1}{8} M \leq r \leq 3 M+\frac{1}{8} M$. In other words, one gets an estimate of the form

$$
\begin{aligned}
\left.\iint_{\mathscr{R}\left(\tau^{\prime}, \tau\right)} r^{-1-\delta} \mathbb{1}_{\left\{|r-3 M| \leq \frac{1}{8} M\right\}}|\not| \Phi\right|^{2} & \\
\leq & C\left(\iint_{\mathscr{R}\left(\tau^{\prime}, \tau\right)}\left(\left.r^{-1-\delta} \mathbb{1}_{\left\{|r-3 M| \geq \frac{1}{8} M\right\}}|\not| \Phi\right|^{2}+r^{-1-\delta}\left(\partial_{r} \Phi\right)^{2}+r^{-1-\delta}\left(\partial_{t^{*}} \Phi\right)^{2}+r^{-3-\delta} \Phi^{2}\right)\right. \\
& \left.\quad+\int_{\Sigma_{\tau}} J_{\mu}^{N}(\Phi) n_{\Sigma_{\tau}}^{\mu}+\int_{\Sigma_{\tau^{\prime}}} J_{\mu}^{N}(\Phi) n_{\Sigma_{\tau^{\prime}}}^{\mu}+\iint_{\mathscr{R}\left(\tau^{\prime}, \tau\right)}\left(\left|\partial_{r} \Phi\right|+\left|r^{-1} \Phi\right|\right)|G|+\iint_{\mathscr{R}\left(\tau^{\prime}, \tau\right)} G^{2}\right) .
\end{aligned}
$$

Using a stability argument, (9) would hold also on Kerr spacetimes. One easily checks that the terms with $G$ on the right-hand side can be estimated in the same manner as before. Hence, the proposition can be proved by applying Proposition 28 .

\section{Vector field multiplier $Z$ and decay of nondegenerate energy}

We follow the definition of $Z$ in [Dafermos and Rodnianski 2008]. Let $Z=u^{2} \underline{L}+v^{2} L$, where $u$ and $v$ are the Schwarzschild coordinates $u=\frac{1}{2}\left(t-r_{S}^{*}\right)$ and $v=\frac{1}{2}\left(t+r_{S}^{*}\right)$, and $\underline{L}=\partial_{u}$ and $L=2 V-\underline{L}$, where $V=\partial_{t^{*}}+\chi(r) a /\left(2 M r_{+}\right) \partial_{\phi^{*}}$ with $\chi$ being a cutoff function that is identically 1 for $r \leq r_{Y}^{-}-\frac{1}{2}\left(r_{Y}^{-}-r_{+}\right)$ and is compactly supported in $\left\{r \leq r_{Y}^{-}-\frac{1}{4}\left(r_{Y}^{-}-r_{+}\right)\right\}$. With this definition, $V$ is Killing except in the set $\left\{r_{Y}^{-}-\frac{1}{2}\left(r_{Y}^{-}-r_{+}\right) \leq r \leq r_{Y}^{-}-\frac{1}{4}\left(r_{Y}^{-}-r_{+}\right)\right\}$. Let $w^{Z}=4 t r_{S}^{*}(1-\mu) / r$. Notice that while $u \rightarrow \infty$ as one approaches the event horizon, $Z$ is continuous up to the event horizon due to the following (however, $Z$ is not $C^{1}$ and hence its deformation tensor is not continuous up to the event horizon):

Proposition 30. In the $\operatorname{Kerr}\left(t^{*}, r, \theta, \phi^{*}\right)$ coordinates,

$$
\underline{L}=(1-\mu) \partial_{t^{*}}-(1-\mu)\left(\frac{2 r_{s}-2 M}{2 r-2 M}\right) \partial_{r} .
$$

In the null frame near the event horizon in Section 2.3, we can write

$$
\underline{L}=\underline{L}^{\hat{V}} \hat{V}+\underline{L}^{\hat{Y}} \hat{Y}+\underline{L}^{A} E_{A}, \quad \text { where }\left|\underline{L}^{\alpha}\right| \leq C(1-\mu) .
$$


Heuristically, we want to show that in the region $\left\{r \geq r_{Y}^{-}\right\}$,

$$
\int_{\Sigma_{\tau} \cap\left\{r \geq r_{Y}^{-}\right\}} J_{\mu}^{Z, w^{Z}}(\Phi) n_{\Sigma_{\tau}}^{\mu} \geq 0
$$

Moreover, we would like to have

$$
\int_{\Sigma_{\tau} \cap\left\{r \geq r_{Y}^{-}\right\}} J_{\mu}^{Z, w^{Z}}(\Phi) n_{\Sigma_{\tau}}^{\mu} \geq \int_{\Sigma_{\tau} \cap\left\{r \geq r_{Y}^{-}\right\}} u^{2}(\underline{L} \Phi)^{2}+v^{2}(L \Phi)^{2}+\left(u^{2}+v^{2}\right)|\not \Phi|^{2}+\left(\frac{u^{2}+v^{2}}{r^{2}}\right) \Phi^{2}
$$

These are true modulo some error terms that can be controlled:

Proposition 31. We have

$$
\begin{aligned}
\int_{\Sigma_{\tau} \cap\left\{r \geq r_{Y}^{-}\right\}} u^{2}(\underline{L} \Phi)^{2}+v^{2}(L \Phi)^{2} & +\left.\left(u^{2}+v^{2}\right)|\not| \Phi\right|^{2}+\left(\frac{u^{2}+v^{2}}{r^{2}}\right) \Phi^{2} \\
& \leq C \int_{\Sigma_{\tau}} J_{\mu}^{Z, w^{Z}}(\Phi) n_{\Sigma_{\tau}}^{\mu}+C \int_{\Sigma_{\tau}} J_{\mu}^{N}(\Phi) n_{\Sigma_{\tau}}^{\mu}+C^{2} \tau^{2} \int_{\Sigma_{\tau} \cap\left\{r \leq r_{Y}^{-}\right\}} J_{\mu}^{N}(\Phi) n_{\Sigma_{\tau}}^{\mu}
\end{aligned}
$$

Proof. The proof is analogous to that in Minkowski spacetime [Morawetz 1975] and Schwarzschild spacetime [Dafermos and Rodnianski 2009]. Recall from the latter that on Schwarzschild spacetime, on a $t$ slice,

$$
\begin{aligned}
\left(J_{S}^{Z, w^{Z}}\right)_{\mu}(\Phi) & n_{\Sigma_{t}}^{\mu} \\
& =\frac{1}{\sqrt{1-\mu}}\left(v^{2}(L \Phi)^{2}+u^{2}(\underline{L} \Phi)^{2}+\left.\left(u^{2}+v^{2}\right)|\not| \Phi\right|^{2}+\frac{2 t r^{*}(1-\mu)}{r} \Phi \partial_{t} \Phi-\frac{r^{*}(1-\mu)}{r} \Phi^{2}\right) .
\end{aligned}
$$

Now, since $t$ and $r^{*}$ are stable under perturbation on $\left\{r \geq r_{Y}^{-}-\left(r_{Y}^{-}-r_{+}\right) / 4\right\}$, we have, on this set,

$$
\begin{array}{r}
\left(J_{K}^{Z, w^{Z}}\right)_{\mu}(\Phi) n_{\Sigma_{\tau}}^{\mu} \geq \frac{1}{\sqrt{1-\mu}}\left(v^{2}(L \Phi)^{2}+u^{2}(\underline{L} \Phi)^{2}+\left(u^{2}+v^{2}\right)|\not \Phi|^{2}+\frac{2 t r^{*}(1-\mu)}{r} \Phi \partial_{t^{*}} \Phi-\frac{r^{*}(1-\mu)}{r} \Phi^{2}\right) \\
-C \epsilon r^{-2}\left(\left(u^{2}+v^{2}\right)(\nabla \Phi)^{2}+t^{*} \Phi^{2}\right)
\end{array}
$$

We now cut off $\Phi$. Define $\hat{\Phi}$ so that it is supported in $\left\{r \geq r_{Y}^{-}-\left(r_{Y}^{-}-r_{+}\right) / 4\right\}$ and equals $\Phi$ in $\left\{r \geq r_{Y}^{-}\right\}$. All the error terms arising from the cutoff will be controlled using the red-shift vector field:

$$
\begin{aligned}
\int_{\Sigma_{\tau}} J_{\mu}^{Z, w^{Z}}(\hat{\Phi}) n_{\Sigma_{\tau}}^{\mu} \geq \int_{\Sigma_{\tau} \cap\left\{r \geq r_{Y}^{-}-\left(r_{Y}^{-}-r_{+}\right) / 4\right\}} \frac{1}{\sqrt{1-\mu}}\left(v^{2}(L \hat{\Phi})^{2}+u^{2}(\underline{L} \hat{\Phi})^{2}+\left(u^{2}+v^{2}\right)|\not \hat{\Phi}|^{2}\right) \\
+\frac{2 t r_{S}^{*}(1-\mu)^{1 / 2}}{r} \hat{\Phi} \partial_{t^{*}} \hat{\Phi}-\frac{r_{S}^{*}(1-\mu)^{1 / 2}}{r} \hat{\Phi}^{2}-C \epsilon r^{-2}\left(\left(u^{2}+v^{2}\right)(\nabla \hat{\Phi})^{2}+t^{*} \hat{\Phi}^{2}\right)
\end{aligned}
$$

The term

$$
\int_{\Sigma_{\tau} \cap\left\{r \geq r_{Y}^{-}-\left(r_{Y}^{-}-r_{+}\right) / 4\right\}} \frac{2 t r_{S}^{*}(1-\mu)^{1 / 2}}{r} \hat{\Phi} \partial_{t^{*}} \hat{\Phi}
$$


is to be handled by two different integrations by parts. Recall [Dafermos and Rodnianski 2009] that on Schwarzschild spacetimes we have

$$
t \partial_{t} \hat{\Phi}=v L \hat{\Phi}+u \underline{L} \hat{\Phi}-r_{S}^{*} \partial_{r_{S}^{*}} \hat{\Phi} \quad \text { and } \quad t \partial_{t} \hat{\Phi}=\frac{t}{r_{S}^{*}}(v L \hat{\Phi}-u \underline{L} \hat{\Phi})-\frac{t^{2}}{r_{S}^{*}} \partial_{r_{S}^{*}} \hat{\Phi}
$$

Therefore, upon integrating by parts, we have on Schwarzschild spacetimes that

$$
\begin{aligned}
\int_{\Sigma_{\tau} \cap\left\{r \geq r_{Y}^{-}-\left(r_{Y}^{-}-r_{+}\right) / 4\right\}} \frac{t r_{S}^{*}(1-\mu)^{1 / 2}}{r} \hat{\Phi} \partial_{t^{*}} \hat{\Phi} \\
=\int_{\Sigma_{\tau} \cap\left\{r \geq r_{Y}^{-}-\left(r_{Y}^{-}-r_{+}\right) / 4\right\}}\left((1-\mu) r^{2} \frac{r_{S}^{*}}{r}(v L \hat{\Phi}+u \underline{L} \hat{\Phi}) \hat{\Phi}+\frac{1}{2} \partial_{r_{S}^{*}}\left((1-\mu) r\left(r^{*}\right)^{2}\right) \hat{\Phi}^{2}\right) d \theta d \phi d r^{*} \\
=\int_{\Sigma_{\tau} \cap\left\{r \geq r_{Y}^{-}-\left(r_{Y}^{-}-r_{+}\right) / 4\right\}}(1-\mu) r^{2}\left(\frac{r_{S}^{*}}{r}(v L \hat{\Phi}+u \underline{L} \hat{\Phi}) \hat{\Phi}+\left(\frac{1}{2} \frac{\left(r_{S}^{*}\right)^{2}}{r^{2}}+\frac{r_{S}^{*}}{r}\right) \hat{\Phi}^{2}\right) d \theta d \phi d r^{*}
\end{aligned}
$$

Notice that in the equation above, we suppressed the volume form in our notation in the first line, while when we write in coordinates as in the second and the third line, we write out the volume form explicitly. Alternatively, we have

$$
\begin{aligned}
\int_{\Sigma_{\tau} \cap\left\{r \geq r_{Y}^{-}-\left(r_{Y}^{-}-r_{+}\right) / 4\right\}} \frac{t r_{S}^{*}(1-\mu)^{1 / 2}}{r} \hat{\Phi} \partial_{t^{*}} \hat{\Phi} \\
=\int_{\Sigma_{\tau} \cap\left\{r \geq r_{Y}^{-}-\left(r_{Y}^{-}-r_{+}\right) / 4\right\}}\left((1-\mu) r^{2} \frac{t^{*}}{r}(v L \hat{\Phi}-u \underline{L} \hat{\Phi}) \hat{\Phi}+\frac{1}{2} \partial_{r^{*}}\left((1-\mu) r\left(t^{*}\right)^{2}\right) \hat{\Phi}^{2}\right) d \theta d \phi d r^{*} \\
=\int_{\Sigma_{\tau} \cap\left\{r \geq r_{Y}^{-}-\left(r_{Y}^{-}-r_{+}\right) / 4\right\}}(1-\mu) r^{2}\left(\frac{t^{*}}{r}(v L \hat{\Phi}-u \underline{L} \hat{\Phi}) \hat{\Phi}+\frac{1}{2} \frac{\left(t^{*}\right)^{2}}{r^{2}} \hat{\Phi}^{2}\right) d \theta d \phi d r^{*} .
\end{aligned}
$$

We would like to imitate this integration by parts on Kerr spacetimes. On the domain of integration, we have

$$
\begin{aligned}
& t \partial_{t} \hat{\Phi}=v L \hat{\Phi}+u \underline{L} \hat{\Phi}-r_{S}^{*} \partial_{r_{S}^{*}} \hat{\Phi} \\
& t \partial_{t} \hat{\Phi}=\frac{t}{r_{S}^{*}}(v L \hat{\Phi}-u \underline{L} \hat{\Phi})-\frac{t^{2}}{r_{S}^{*}} \partial_{r_{S}^{*}} \hat{\Phi}
\end{aligned}
$$

The volume form on a constant $t^{*}$ slice on a Kerr spacetime is close to that on a Schwarzschild spacetime, including in the region being considered. In other words, for $r \geq r_{Y}^{-}-\left(r_{Y}^{-}-r_{+}\right) / 4$,

$$
d \operatorname{Vol}_{\Sigma_{\tau}}=\left(r^{2}(1-\mu)^{-1 / 2}+O_{1}(\epsilon)\right) d r d x^{A} d x^{B}
$$

Moreover, for $r \geq r_{Y}^{-}-\left(r_{Y}^{-}-r_{+}\right) / 4$,

$$
\partial_{r_{S}^{*}}=\left((1-\mu)+O_{1}\left(\epsilon r^{-2}\right)\right) \partial_{r} .
$$


Therefore, using (10), we have

$$
\begin{aligned}
& \int_{\Sigma_{\tau} \cap\left\{r \geq r_{Y}^{-}-\left(r_{Y}^{-}-r_{+}\right) / 4\right\}} \frac{t r_{S}^{*}(1-\mu)^{1 / 2}}{r} \hat{\Phi} \partial_{t^{*}} \hat{\Phi} \\
& =\int_{\Sigma_{\tau} \cap\left\{r \geq r_{Y}^{-}-\left(r_{Y}^{-}-r_{+}\right) / 4\right\}}\left(\left(r r_{S}^{*}+O(\epsilon)\right)(v L \hat{\Phi}+u \underline{L} \hat{\Phi}) \hat{\Phi}+\left(\frac{1}{2} \partial_{r}\left((1-\mu) r\left(r_{S}^{*}\right)^{2}\right)+O(\epsilon)\right) \hat{\Phi}^{2}\right) d r d x^{A} d x^{B} \\
& =\int_{\Sigma_{\tau} \cap\left\{r \geq r_{Y}^{-}-\left(r_{Y}^{-}-r_{+}\right) / 4\right\}}(1-\mu)^{1 / 2}\left(\left(\frac{r_{S}^{*}}{r}+O\left(\epsilon r^{-2}\right)\right)(v L \hat{\Phi}+u \underline{L} \hat{\Phi}) \hat{\Phi}+\left(\frac{1}{2} \frac{\left(r_{S}^{*}\right)^{2}}{r^{2}}+\frac{r_{S}^{*}}{r}+O\left(\epsilon r^{-2}\right)\right) \hat{\Phi}^{2}\right) .
\end{aligned}
$$

Alternatively, we can integrate by parts after using (11):

$$
\begin{aligned}
& \int_{\Sigma_{\tau} \cap\left\{r \geq r_{Y}^{-}-\left(r_{Y}^{-}-r_{+}\right) / 4\right\}} \frac{t r_{S}^{*}(1-\mu)^{1 / 2}}{r} \hat{\Phi} \partial_{t^{*}} \hat{\Phi} \\
& =\int_{\Sigma_{\tau} \cap\left\{r \geq r_{Y}^{-}-\left(r_{Y}^{-}-r_{+}\right) / 4\right\}}\left(\left(r t^{*}+O(\epsilon)\right)(v L \hat{\Phi}-u \underline{L} \hat{\Phi}) \hat{\Phi}+\left(\frac{1}{2} \partial_{r}\left((1-\mu) r\left(t^{*}\right)^{2}\right)+O(\epsilon)\right) \hat{\Phi}^{2}\right) \\
& =\int_{\Sigma_{\tau} \cap\left\{r \geq r_{Y}^{-}-\left(r_{Y}^{-}-r_{+}\right) / 4\right\}}(1-\mu)^{1 / 2}\left(\left(\frac{t^{*}}{r}+O\left(\epsilon r^{-2}\right)\right)(v L \hat{\Phi}-u \underline{L} \hat{\Phi}) \hat{\Phi}+\left(\frac{1}{2} \frac{\left(t^{*}\right)^{2}}{r^{2}}+O\left(\epsilon r^{-2}\right)\right) \hat{\Phi}^{2}\right) .
\end{aligned}
$$

Therefore, we have

$$
\begin{aligned}
& \int_{\Sigma_{\tau}} J_{\mu}^{Z, w^{Z}}(\hat{\Phi}) n_{\Sigma_{\tau}}^{\mu} \\
& \geq \int_{\Sigma_{\tau} \cap\left\{r \geq r_{Y}^{-}-\left(r_{Y}^{-}-r_{+}\right) / 4\right\}} \frac{1}{\sqrt{1-\mu}}\left(v^{2}(L \hat{\Phi})^{2}+u^{2}(\underline{L} \hat{\Phi})^{2}+\left(u^{2}+v^{2}\right)|\not \hat{\Phi}|^{2}\right) \\
& +\frac{2 t r_{S}^{*}(1-\mu)^{1 / 2}}{r} \hat{\Phi} \partial_{t^{*}} \hat{\Phi}-\frac{r_{S}^{*}(1-\mu)^{1 / 2}}{r} \hat{\Phi}^{2}-C \epsilon r^{-2}\left(\left(u^{2}+v^{2}\right)(D \hat{\Phi})^{2}+t^{*} \hat{\Phi}^{2}\right) \\
& \geq \int_{\Sigma_{\tau} \cap\left\{r \geq r_{Y}^{-}-\left(r_{Y}^{-}-r_{+}\right) / 4\right\}} \frac{1}{\sqrt{1-\mu}}\left(v^{2}(L \hat{\Phi})^{2}+u^{2}(\underline{L} \hat{\Phi})^{2}+\left(u^{2}+v^{2}\right)|\not \hat{\Phi}|^{2}\right) \\
& +\frac{r_{S}^{*}(1-\mu)^{1 / 2}}{r}(v L \hat{\Phi}+u \underline{L} \hat{\Phi}) \hat{\Phi}+\frac{1}{2} \frac{\left(r_{S}^{*}\right)^{2}(1-\mu)^{1 / 2}}{r^{2}} \hat{\Phi}^{2} \\
& +\frac{t^{*}(1-\mu)^{1 / 2}}{r}(v L \hat{\Phi}-u \underline{L} \hat{\Phi}) \hat{\Phi}+\frac{1}{2} \frac{\left(t^{*}\right)^{2}}{r^{2}} \hat{\Phi}^{2}-C \epsilon r^{-2}\left(\left(u^{2}+v^{2}\right)(D \hat{\Phi})^{2}+t^{*} \hat{\Phi}^{2}\right) \\
& \geq c\left(\int_{\Sigma_{\tau} \cap\left\{r \geq r_{Y}^{-}-\left(r_{Y}^{-}-r_{+}\right) / 4\right\}} \mu\left((v L \hat{\Phi}+u \underline{L} \hat{\Phi})^{2}+(v L \hat{\Phi}-u \underline{L} \hat{\Phi})^{2}\right)\right.
\end{aligned}
$$

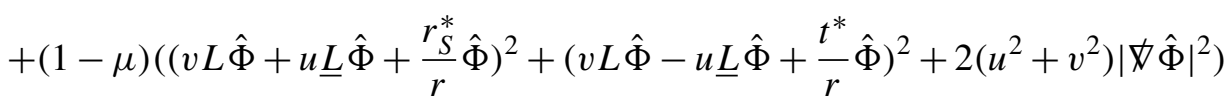

$$
\begin{aligned}
& \left.-C \epsilon r^{-2}\left(\left(u^{2}+v^{2}\right)(D \hat{\Phi})^{2}+t^{*} \hat{\Phi}^{2}\right)\right) \text {, }
\end{aligned}
$$

where the last line is obtained by first completing the square and using $c \leq 1-\mu \leq C$ in this region of $r$. Let us for now ignore the error term and look at the other terms (which are manifestly positive). By exactly the same argument as in [Dafermos and Rodnianski 2009], these positive terms provide good 
estimates:

$$
\begin{gathered}
\int_{\Sigma_{\tau} \cap\left\{r \geq r_{Y}^{-}-\left(r_{Y}^{-}-r_{+}\right) / 4\right\}} \mu\left((v L \hat{\Phi}+u \underline{L} \hat{\Phi})^{2}+(v L \hat{\Phi}-u \underline{L} \hat{\Phi})^{2}\right) \\
+(1-\mu)\left(\left(v L \hat{\Phi}+u \underline{L} \hat{\Phi}+\frac{r_{S}^{*}}{r} \hat{\Phi}\right)^{2}+\left(v L \hat{\Phi}-u \underline{L} \hat{\Phi}+\frac{t^{*}}{r} \hat{\Phi}\right)^{2}+\left.2\left(u^{2}+v^{2}\right)|\not| \hat{\Phi}\right|^{2}\right) \\
\geq c \int_{\Sigma_{\tau} \cap\left\{r \geq r_{Y}^{-}-\left(r_{Y}^{-}-r_{+}\right) / 4\right\}}\left(v^{2}(L \hat{\Phi})^{2}+u^{2}(\underline{L} \hat{\Phi})^{2}+\left(u^{2}+v^{2}\right)|\not \Phi \hat{\Phi}|^{2}+\frac{u^{2}+v^{2}}{r^{2}} \hat{\Phi}^{2}\right) \\
+C \epsilon \int_{\Sigma_{\tau} \cap\left\{r \geq r_{Y}^{-}-\left(r_{Y}^{-}-r_{+}\right) / 4\right\}} r^{-2}\left(\left(u^{2}+v^{2}\right)(D \hat{\Phi})^{2}+t^{*} \hat{\Phi}^{2}\right) .
\end{gathered}
$$

See [Dafermos and Rodnianski 2009] for the proof. This together with $J_{\mu}^{N}(\hat{\Phi}) n_{\Sigma_{\tau}}^{\mu}$ bounds the error term in (12):

$$
\begin{gathered}
\int_{\Sigma_{\tau} \cap\left\{r \geq r_{Y}^{-}-\left(r_{Y}^{-}-r_{+}\right) / 4\right\}} r^{-2}\left(\left(u^{2}+v^{2}\right)(D \hat{\Phi})^{2}+\tau \Phi^{2}\right) \\
\leq C \int_{\Sigma_{\tau} \cap\left\{r \geq r_{Y}^{-}-\left(r_{Y}^{-}-r_{+}\right) / 4\right\}}\left(v^{2}(L \hat{\Phi})^{2}+u^{2}(\underline{L} \hat{\Phi})^{2}+\left.\left(u^{2}+v^{2}\right)|\not| \hat{\Phi}\right|^{2}+\frac{u^{2}+v^{2}}{r^{2}} \hat{\Phi}^{2}\right) \\
\quad+C \int_{\Sigma_{\tau} \cap\{r \geq \tau / 4\}}(\underline{L} \hat{\Phi})^{2} \\
\leq C \int_{\Sigma_{\tau} \cap\left\{r \geq r_{Y}^{-}-\left(r_{Y}^{-}-r_{+}\right) / 4\right\}}\left(v^{2}(L \hat{\Phi})^{2}+u^{2}(\underline{L} \hat{\Phi})^{2}+\left.\left(u^{2}+v^{2}\right)|\not| \hat{\Phi}\right|^{2}+\frac{u^{2}+v^{2}}{r^{2}} \hat{\Phi}^{2}\right) \\
\quad+C \int_{\Sigma_{\tau}} J_{\mu}^{N}(\hat{\Phi}) n_{\Sigma_{\tau}}^{\mu} \\
\quad \mu\left((v L \hat{\Phi}+u \underline{L} \hat{\Phi})^{2}+(v L \hat{\Phi}-u \underline{L} \hat{\Phi})^{2}\right)+\frac{\left.\left.\left.t^{*} \hat{\Phi}\right)^{2}+2\left(u^{2}+v^{2}\right)|\not \hat{\Phi}|^{2}\right)\right)}{r}+\int_{\Sigma_{\tau} \cap\left\{r \geq r_{Y}^{-}-\left(r_{Y}^{-}-r_{+}\right) / 4\right\}}+C \int_{\Sigma_{\tau}} J_{\mu}^{N}(\hat{\Phi}) n_{\Sigma_{\tau}}^{\mu} .
\end{gathered}
$$

Therefore, if $\epsilon$ is chosen to be small enough, then (12) implies that

$$
\begin{aligned}
\int_{\Sigma_{\tau}} J_{\mu}^{Z, w^{Z}}(\hat{\Phi}) n_{\Sigma_{\tau}}^{\mu} & +\int_{\Sigma_{\tau}} J_{\mu}^{N}(\hat{\Phi}) n_{\Sigma_{\tau}}^{\mu} \\
& \geq c \int_{\Sigma_{\tau} \cap\left\{r \geq r_{Y}^{-}-\left(r_{Y}^{-}-r_{+}\right) / 4\right\}}\left(v^{2}(L \hat{\Phi})^{2}+u^{2}(\underline{L} \hat{\Phi})^{2}+\left.\left(u^{2}+v^{2}\right)|\not| \hat{\Phi}\right|^{2}+\frac{u^{2}+v^{2}}{r^{2}} \hat{\Phi}^{2}\right) .
\end{aligned}
$$

We note that $c$ here is independent of the choice of $r_{Y}^{-}$. With this bound we would like to estimate $\int_{\mathbb{S}^{2}} \Phi(\tau, r)^{2}$. Using (13), there exists a $\tilde{r} \in\left[r_{Y}^{-}, r_{Y}^{-}+1\right]$ such that

$$
\int_{\mathbb{S}^{2}} \Phi(\tau, \tilde{r})^{2}=\int_{\mathbb{S}^{2}} \hat{\Phi}(\tau, \tilde{r})^{2} \leq C \tau^{-2}\left(\int_{\Sigma_{\tau}} J_{\mu}^{Z, w^{Z}}(\hat{\Phi}) n_{\Sigma_{\tau}}^{\mu}+\int_{\Sigma_{\tau}} J_{\mu}^{N}(\hat{\Phi}) n_{\Sigma_{\tau}}^{\mu}\right) \text {. }
$$


Then for every $r \in\left[r_{+}, r_{Y}^{-}\right]$, since $\Phi(\tau, \tilde{r})-\Phi(\tau, r)=\int_{r}^{\tilde{r}} \partial_{r} \Phi d r$, we have

$$
\begin{aligned}
\int_{\mathbb{S}^{2}} \Phi(\tau, r)^{2} & \leq \int_{\mathbb{S}^{2}} \Phi(\tau, \tilde{r})^{2}+(\tilde{r}-r) \int_{\Sigma_{\tau} \cap[r, \tilde{r}]} J_{\mu}^{N}(\Phi) n_{\Sigma_{\tau}}^{\mu} \\
& \leq C \int_{\Sigma_{\tau} \cap\left\{r \leq r_{Y}^{-}\right\}} J_{\mu}^{N}(\Phi) n_{\Sigma_{\tau}}^{\mu}+C \tau^{-2}\left(\int_{\Sigma_{\tau}} J_{\mu}^{Z, w^{Z}}(\hat{\Phi}) n_{\Sigma_{\tau}}^{\mu}+\int_{\Sigma_{\tau}} J_{\mu}^{N}(\hat{\Phi}) n_{\Sigma_{\tau}}^{\mu}\right)
\end{aligned}
$$

Now we need to obtain estimates for $\Phi$ from that for $\hat{\Phi}$. It is obvious that

$$
\begin{aligned}
\int_{\Sigma_{\tau} \cap\left\{r \geq r_{Y}^{-}\right\}} & \left(v^{2}(L \Phi)^{2}+u^{2}(\underline{L} \Phi)^{2}+\left.\left(u^{2}+v^{2}\right)|\not| \Phi\right|^{2}+\frac{u^{2}+v^{2}}{r^{2}} \Phi^{2}\right) \\
& \leq \int_{\Sigma_{\tau} \cap\left\{r \geq r_{Y}^{-}\right\}}\left(v^{2}(L \hat{\Phi})^{2}+u^{2}(\underline{L} \hat{\Phi})^{2}+\left.\left(u^{2}+v^{2}\right)|\not| \hat{\Phi}\right|^{2}+\frac{u^{2}+v^{2}}{r^{2}} \hat{\Phi}^{2}\right) \\
& \leq \int_{\Sigma_{\tau} \cap\left\{r \geq r_{Y}^{-}-\left(r_{Y}^{-}-r_{+}\right) / 4\right\}}\left(v^{2}(L \hat{\Phi})^{2}+u^{2}(\underline{L} \hat{\Phi})^{2}+\left(u^{2}+v^{2}\right)|\not \hat{\Phi}|^{2}+\frac{u^{2}+v^{2}}{r^{2}} \hat{\Phi}^{2}\right),
\end{aligned}
$$

and

$$
\begin{aligned}
& \int_{\Sigma_{\tau}} J_{\mu}^{Z, w^{Z}}(\hat{\Phi}) n_{\Sigma_{\tau}}^{\mu}+\int_{\Sigma_{\tau}} J_{\mu}^{N}(\hat{\Phi}) n_{\Sigma_{\tau}}^{\mu} \\
& \quad \leq \int_{\Sigma_{\tau}} J_{\mu}^{Z, w^{Z}}(\Phi) n_{\Sigma_{\tau}}^{\mu}+\int_{\Sigma_{\tau}} J_{\mu}^{N}(\Phi) n_{\Sigma_{\tau}}^{\mu}+C \tau^{2} \int_{\Sigma_{\tau} \cap\left\{r \leq r_{Y}^{-}\right\}} J_{\mu}^{N}(\Phi) n_{\Sigma_{\tau}}^{\mu}+C \tau^{2} \int_{\Sigma_{\tau} \cap\left\{r \leq r_{Y}^{-}\right\}} \Phi^{2}
\end{aligned}
$$

(where we have used Proposition 30 to show that the $u^{2}$ factor comes with a factor of $1-\mu$ )

$$
\begin{aligned}
& \leq \int_{\Sigma_{\tau}} J_{\mu}^{Z, w^{Z}}(\Phi) n_{\Sigma_{\tau}}^{\mu}+\int_{\Sigma_{\tau}} J_{\mu}^{N}(\Phi) n_{\Sigma_{\tau}}^{\mu}+C \tau^{2} \int_{\Sigma_{\tau} \cap\left\{r \leq r_{Y}^{-}\right\}} J_{\mu}^{N}(\Phi) n_{\Sigma_{\tau}}^{\mu} \\
&+C\left(r_{Y}^{-}-r_{+}\right)\left(\int_{\Sigma_{\tau}} J_{\mu}^{Z, w^{Z}}(\hat{\Phi}) n_{\Sigma_{\tau}}^{\mu}+\int_{\Sigma_{\tau}} J_{\mu}^{N}(\hat{\Phi}) n_{\Sigma_{\tau}}^{\mu}\right) \\
& \leq \int_{\Sigma_{\tau}} J_{\mu}^{Z, w^{Z}}(\Phi) n_{\Sigma_{\tau}}^{\mu}+\int_{\Sigma_{\tau}} J_{\mu}^{N}(\Phi) n_{\Sigma_{\tau}}^{\mu}+C \tau^{2} \int_{\Sigma_{\tau} \cap\left\{r \leq r_{Y}^{-}\right\}} J_{\mu}^{N}(\Phi) n_{\Sigma_{\tau}}^{\mu} \\
&+\frac{1}{2}\left(\int_{\Sigma_{\tau}} J_{\mu}^{Z, w^{Z}}(\hat{\Phi}) n_{\Sigma_{\tau}}^{\mu}+\int_{\Sigma_{\tau}} J_{\mu}^{N}(\hat{\Phi}) n_{\Sigma_{\tau}}^{\mu}\right)
\end{aligned}
$$

for $r_{Y}^{-}$chosen to be sufficiently close to $r_{+}$. Then

$$
\begin{aligned}
\int_{\Sigma_{\tau}} J_{\mu}^{Z, w^{Z}}(\hat{\Phi}) n_{\Sigma_{\tau}}^{\mu}+\int_{\Sigma_{\tau}} J_{\mu}^{N}(\hat{\Phi}) & n_{\Sigma_{\tau}}^{\mu} \\
\leq & \int_{\Sigma_{\tau}} J_{\mu}^{Z, w^{Z}}(\Phi) n_{\Sigma_{\tau}}^{\mu}+\int_{\Sigma_{\tau}} J_{\mu}^{N}(\Phi) n_{\Sigma_{\tau}}^{\mu}+C \tau^{2} \int_{\Sigma_{\tau} \cap\left\{r \leq r_{Y}^{-}\right\}} J_{\mu}^{N}(\Phi) n_{\Sigma_{\tau}}^{\mu} .
\end{aligned}
$$

Remark. From this point onward, we consider $r_{Y}^{-}$to be fixed. We note again that $r_{Y}^{-}$is chosen so that (5) and (15) hold. 
Remark. The proof of the proposition above in particular shows that

$$
\int_{\Sigma_{\tau}} J_{\mu}^{Z, w^{Z}}(\Phi) n_{\Sigma_{\tau}}^{\mu}+C \tau^{2} \int_{\Sigma_{\tau} \cap\left\{r \leq r_{Y}^{-}\right\}} J_{\mu}^{N}(\Phi) n_{\Sigma_{\tau}}^{\mu} \geq 0
$$

To use this proposition, it is helpful to have a localized version of $\Phi$. This follows [Dafermos and Rodnianski 2008; 2009]. The idea is to use the finite speed of propagation and cutoff $\Phi$ outside the domain of dependence. Focus now on the time interval $\left[\tau^{\prime}, \tau\right]$. Take $\tilde{G}$ to be any smooth function agreeing with $G$ on the domain of dependence of the region $\left(t^{*}=\tau, r \leq \tau / 2\right)$. Let $\tilde{\Phi}\left(\tau^{\prime}\right)=\chi \Phi\left(\tau^{\prime}\right)$ and $\partial_{t^{*}} \tilde{\Phi}\left(\tau^{\prime}\right)=\chi \partial_{t^{*}} \Phi\left(\tau^{\prime}\right)$, where $\chi$ is a cutoff function identically equal to 1 for $r \leq \frac{7}{10} \tau^{\prime}$ and compactly supported in $r \leq \frac{9}{10} \tau^{\prime}$. The region for which $\chi$ is one is inside the domain of dependence of the region $\left(t^{*}=\tau, r \leq \tau / 2\right)$ if $\tau^{\prime} \leq \tau \leq(1.1) \tau^{\prime}$. We solve for $\square_{g_{K}} \tilde{\Phi}=\tilde{G}$.

With this definition of $\tilde{\Phi}$, we have two ways to estimate the nondegenerate energy of $\tilde{\Phi}$ :

Proposition 32. We have

$$
\begin{aligned}
& \int_{\Sigma_{\tau^{\prime}}} J_{\mu}^{N}(\tilde{\Phi}) n_{\Sigma_{\tau^{\prime}}}^{\mu} \leq C \int_{\Sigma_{\tau^{\prime}}} J_{\mu}^{N}(\Phi) n_{\Sigma_{\tau^{\prime}}}^{\mu}, \\
& \int_{\Sigma_{\tau^{\prime}}} J_{\mu}^{N}(\tilde{\Phi}) n_{\Sigma_{\tau^{\prime}}}^{\mu} \leq C^{2} \int_{\Sigma_{\tau^{\prime}} \cap\left\{r \leq r_{Y}^{-}\right\}} J_{\mu}^{N}(\Phi) n_{\Sigma_{\tau^{\prime}}}^{\mu}+C\left(\tau^{\prime}\right)^{-2}\left(\int_{\Sigma_{\tau^{\prime}}} J_{\mu}^{N}(\Phi) n_{\Sigma_{\tau^{\prime}}}^{\mu}+\int_{\Sigma_{\tau^{\prime}}} J_{\mu}^{Z, w^{Z}}(\Phi) n_{\Sigma_{\tau^{\prime}}}^{\mu}\right) .
\end{aligned}
$$

Proof. The first part is an easy application of Proposition 21:

$$
\begin{aligned}
\int_{\Sigma_{\tau^{\prime}}} J_{\mu}^{N}(\tilde{\Phi}) n_{\Sigma_{\tau^{\prime}}}^{\mu} & \leq C \int_{\Sigma_{\tau^{\prime}} \cap\left\{R \leq r \leq \frac{9}{10} \tau^{\prime}\right\}}\left((D \Phi)^{2}+\left(\tau^{\prime}\right)^{-2} \Phi^{2}\right) \\
& \leq C \int_{\Sigma_{\tau^{\prime}} \cap\left\{R \leq r \leq \frac{9}{10} \tau^{\prime}\right\}}\left((D \Phi)^{2}+r^{-2} \Phi^{2}\right) \leq C \int_{\Sigma_{\tau^{\prime}}} J_{\mu}^{N}(\Phi) n_{\Sigma_{\tau^{\prime}}}^{\mu} .
\end{aligned}
$$

Following (14), we have

$$
\int_{\Sigma_{\tau^{\prime}} \cap\left\{r \leq r_{Y}^{-}\right\}} \Phi^{2} \leq C\left(\int_{\Sigma_{\tau^{\prime}} \cap\left\{r \leq r_{Y}^{-}\right\}} J_{\mu}^{N}(\Phi) n_{\Sigma_{\tau^{\prime}}}^{\mu}+\int_{\Sigma_{\tau^{\prime}} \cap\left\{r_{Y}^{-} \leq r \leq r_{Y}^{+}\right\}} \Phi^{2}\right) .
$$

Using this and Proposition 31, we have

$$
\begin{aligned}
\int_{\Sigma_{\tau^{\prime}}} J_{\mu}^{N}(\tilde{\Phi}) n_{\Sigma_{\tau^{\prime}}}^{\mu} & \\
\leq & C \int_{\Sigma_{\tau^{\prime}} \cap\left\{r \leq \frac{9}{10} \tau^{\prime}\right\}}\left((D \Phi)^{2}+\left(\tau^{\prime}\right)^{-2} \Phi^{2}\right) \\
\leq & C \int_{\Sigma_{\tau^{\prime}} \cap\left\{r \leq r_{Y}^{-}\right\}}\left((D \Phi)^{2}+\Phi^{2}\right) \\
& \quad+C\left(\tau^{\prime}\right)^{-2} \int_{\Sigma_{\tau^{\prime}} \cap\left\{r_{Y}^{-} \leq r \leq \frac{9}{10} \tau^{\prime}\right\}}\left(u^{2}(\underline{L} \Phi)^{2}+v^{2}(L \Phi)^{2}+\left(u^{2}+v^{2}\right)|\not \Phi|^{2}+\left(\frac{u^{2}+v^{2}}{r^{2}}\right) \Phi^{2}\right) \\
& \leq C^{2} \int_{\Sigma_{\tau^{\prime}} \cap\left\{r \leq r_{Y}^{-}\right\}} J_{\mu}^{N}(\Phi) n_{\Sigma_{\tau^{\prime}}}^{\mu}+C\left(\tau^{\prime}\right)^{-2}\left(\int_{\Sigma_{\tau^{\prime}}} J_{\mu}^{N}(\Phi) n_{\Sigma_{\tau^{\prime}}}^{\mu}+\int_{\Sigma_{\tau^{\prime}}} J_{\mu}^{Z, w^{Z}}(\Phi) n_{\Sigma_{\tau^{\prime}}}^{\mu}\right) .
\end{aligned}
$$


The cutoff procedure above will also allow us to localize the estimates for the bulk term:

Proposition 33. Let $G=G_{1}+G_{2}$ be any way to decompose the function $G$. Then for $\tau^{\prime} \leq \tau \leq(1.1) \tau^{\prime}$, we have

(1) the localized boundedness estimate

$$
\begin{gathered}
\int_{\Sigma_{\tau} \cap\{r \leq \tau / 2\}} J_{\mu}^{N}(\Phi) n_{\Sigma_{\tau}}^{\mu}+\int_{\mathscr{H}\left(\tau^{\prime}, \tau\right)} J_{\mu}^{N}(\Phi) n_{\mathscr{R ^ { + }}}^{\mu}+\iint_{\mathscr{R}\left(\tau^{\prime}, \tau\right) \cap\left\{r \leq r_{Y}^{-}\right\}} K^{N}(\Phi)+\iint_{\mathscr{R}\left(\tau^{\prime}, \tau\right) \cap\left\{r \leq \frac{1}{2} t^{*}\right\}} K^{X_{0}}(\Phi) \\
\leq C\left(\int_{\Sigma_{\tau^{\prime}}} J_{\mu}^{N}(\Phi) n_{\Sigma_{\tau^{\prime}}}^{\mu}+\left(\int_{\tau^{\prime}-1}^{\tau+1}\left(\int_{\Sigma_{t^{*}} \cap\left\{r \leq \frac{9}{10} t^{*}\right\}} G_{1}^{2}\right)^{1 / 2} d t^{*}\right)^{2}\right. \\
+\iint_{\mathscr{R}\left(\tau^{\prime}-1, \tau+1\right) \cap\left\{r \leq \frac{9}{10} *^{*}\right\}} G_{1}^{2}+\sum_{m=0}^{1} \iint_{\mathscr{R}\left(\tau^{\prime}-1, \tau+1\right) \cap\left\{r \leq \frac{9}{10} t^{*}\right\}} r^{1+\delta}\left(\partial_{t^{*}}^{m} G_{2}\right)^{2} \\
\left.+\sup _{t^{*} \in\left[\tau^{\prime}-1, \tau+1\right]} \int_{\Sigma_{t^{*}} \cap\left\{|r-3 M| \leq \frac{1}{8} M\right\} \cap\left\{r \leq \frac{9}{10} t^{*}\right\}} G_{2}{ }^{2}\right) ;
\end{gathered}
$$

(2) the localized decay estimate

$$
\begin{aligned}
& \int_{\Sigma_{\tau} \cap\left\{r \leq \frac{1}{2} \tau\right\}} J_{\mu}^{N}(\Phi) n_{\Sigma_{\tau}}^{\mu}+\int_{\mathscr{H}\left(\tau^{\prime}, \tau\right)} J_{\mu}^{N}(\Phi) n_{\mathscr{H}^{+}}^{\mu}+\iint_{\mathscr{R}\left(\tau^{\prime}, \tau\right) \cap\left\{r \leq r_{Y}^{-}\right\}} K^{N}(\Phi)+\iint_{\mathscr{R}\left(\tau^{\prime}, \tau\right) \cap\left\{r \leq \frac{1}{2} t^{*}\right\}} K^{X_{0}}(\Phi) \\
& \leq C\left(\tau^{-2} \int_{\Sigma_{\tau^{\prime}}} J_{\mu}^{Z+N, w^{Z}}(\Phi) n_{\Sigma_{\tau^{\prime}}}^{\mu}+C \int_{\Sigma_{\tau^{\prime}} \cap\left\{r \leq r_{Y}^{-}\right\}} J_{\mu}^{N}(\Phi) n_{\Sigma_{\tau^{\prime}}}^{\mu}\right) \\
& +C\left(\left(\int_{\tau^{\prime}-1}^{\tau+1}\left(\int_{\Sigma_{t^{*} \cap\left\{r \leq \frac{9}{10} t^{*}\right\}}} G_{1}^{2}\right)^{1 / 2} d t^{*}\right)^{2}+\iint_{\mathscr{R}\left(\tau^{\prime}-1, \tau+1\right) \cap\left\{r \leq \frac{9}{10} t^{*}\right\}} G_{1}^{2}\right. \\
& \left.\quad+\sum_{m=0}^{1} \iint_{\mathscr{R}\left(\tau^{\prime}-1, \tau+1\right) \cap\left\{r \leq \frac{9}{10} *^{*}\right\}} r^{1+\delta}\left(\partial_{t^{*}}^{m} G_{2}\right)^{2}+\sup _{t^{*} \in\left[\tau^{\prime}-1, \tau+1\right]} \int_{\Sigma_{t^{*}} \cap\left\{|r-3 M| \leq \frac{1}{8} M\right\} \cap\left\{r \leq \frac{9}{10} t^{*}\right\}} G_{2}^{2}\right) .
\end{aligned}
$$

Proof. Applying Proposition 28 to the equation $\square_{g_{K}} \tilde{\Phi}=\tilde{G}$, we have

$$
\begin{gathered}
\int_{\Sigma_{\tau} \cap\left\{r \leq \frac{1}{2} \tau\right\}} J_{\mu}^{N}(\tilde{\Phi}) n_{\Sigma_{\tau}}^{\mu}+\int_{\mathscr{H}\left(\tau^{\prime}, \tau\right)} J_{\mu}^{N}(\tilde{\Phi}) n_{\mathscr{H}+}^{\mu}+\iint_{\mathscr{R}\left(\tau^{\prime}, \tau\right) \cap\left\{r \leq r_{Y}^{-}\right\}} K^{N}(\tilde{\Phi})+\iint_{\mathscr{R}\left(\tau^{\prime}, \tau\right) \cap\left\{r \leq \frac{1}{2} t^{*}\right\}} K^{X_{0}}(\tilde{\Phi}) \\
\leq C\left(\int_{\Sigma_{\tau^{\prime}}} J_{\mu}^{N}(\tilde{\Phi}) n_{\Sigma_{\tau^{\prime}}}^{\mu}+\left(\int_{\tau^{\prime}-1}^{\tau+1}\left(\int_{\Sigma_{t^{*}}} \tilde{G}_{1}^{2}\right)^{1 / 2} d t^{*}\right)^{2}+\iint_{\mathscr{R}\left(\tau^{\prime}-1, \tau+1\right)} \tilde{G}_{1}^{2}\right. \\
+\sum_{m=0}^{1} \iint_{\mathscr{R}\left(\tau^{\prime}-1, \tau+1\right)} r^{1+\delta}\left(\partial_{t^{*}}^{m} \tilde{G}_{2}\right)^{2}+\sup _{t^{*} \in\left[\tau^{\prime}-1, \tau+1\right]} \int_{\left.\Sigma_{t^{*} \cap\left\{\left\{r-3 M \mid \leq \frac{1}{8} M\right\}\right.} \tilde{G}_{2}^{2}\right) .}
\end{gathered}
$$


Since by the finite speed of propagation, $\tilde{\Phi}=\Phi$ in $\left\{r \leq \frac{1}{2} t^{*}\right\}$, we have

$$
\begin{gathered}
\int_{\Sigma_{\tau} \cap\left\{r \leq \frac{1}{2} \tau\right\}} J_{\mu}^{N}(\Phi) n_{\Sigma_{\tau}}^{\mu}+\int_{\mathscr{H}\left(\tau^{\prime}, \tau\right)} J_{\mu}^{N}(\Phi) n_{\mathscr{\mathscr { H } ^ { + }}}^{\mu}+\iint_{\mathscr{R}^{\prime}\left(\tau^{\prime}, \tau\right) \cap\left\{r \leq r_{Y}^{-}\right\}} K^{N}(\Phi)+\iint_{\mathscr{R}^{\prime}\left(\tau^{\prime}, \tau\right) \cap\left\{r \leq \frac{1}{2} t^{*}\right\}} K^{X_{0}}(\Phi) \\
\leq C\left(\int_{\Sigma_{\tau^{\prime}}} J_{\mu}^{N}(\tilde{\Phi}) n_{\Sigma_{\tau^{\prime}}}^{\mu}+\left(\int_{\tau^{\prime}-1}^{\tau+1}\left(\int_{\Sigma_{t^{*}}} \tilde{G}_{1}^{2}\right)^{1 / 2} d t^{*}\right)^{2}+\iint_{\mathscr{R}\left(\tau^{\prime}-1, \tau+1\right)} \tilde{G}_{1}^{2}\right. \\
\left.\quad+\sum_{m=0}^{1} \iint_{\mathscr{R}\left(\tau^{\prime}-1, \tau+1\right)} r^{1+\delta}\left(\partial_{t^{*}}^{m} \tilde{G}_{2}\right)^{2}+\sup _{t^{*} \in\left[\tau^{\prime}-1, \tau+1\right]} \int_{\Sigma_{t^{*}} \cap\left\{|r-3 M| \leq \frac{1}{8} M\right\}} \tilde{G}_{2}^{2}\right) .
\end{gathered}
$$

Now, we choose a particular $\tilde{G}$. Define $\tilde{G}$ to be $G$ for $r \leq \frac{7}{10} t^{*}$, and 0 for $r \geq \frac{9}{10} t^{*}$. It can be easily shown that one has the bounds

$$
\left|\partial_{t^{*}}^{m} \tilde{G}\right| \leq C \sum_{k=0}^{m}\left|\left(\frac{r^{*}}{\left(t^{*}\right)^{2}}\right)^{k} \partial_{t^{*}}^{m-k} G\right| \leq C \sum_{k=0}^{m}\left|\left(t^{*}\right)^{-k} \partial_{t^{*}}^{m-k} G\right| \quad \text { for } \frac{7}{10} t^{*} \leq r \leq \frac{9}{10} t^{*} .
$$

Therefore, we have

$$
\begin{aligned}
& \int_{\Sigma_{\tau} \cap\left\{r \leq \frac{1}{2} \tau\right\}} J_{\mu}^{N}(\Phi) n_{\Sigma_{\tau}}^{\mu}+\int_{\mathscr{H}\left(\tau^{\prime}, \tau\right)} J_{\mu}^{N}(\Phi) n_{\mathscr{H} t^{+}}^{\mu}+\iint_{\mathscr{R}\left(\tau^{\prime}, \tau\right) \cap\left\{r \leq r_{Y}^{-}\right\}} K^{N}(\Phi)+\iint_{\mathscr{R}\left(\tau^{\prime}, \tau\right) \cap\left\{r \leq \frac{1}{2} *^{*}\right\}} K^{X_{0}}(\Phi) \\
& \leq C\left(\int_{\Sigma_{\tau^{\prime}}} J_{\mu}^{N}(\tilde{\Phi}) n_{\Sigma_{\tau^{\prime}}}^{\mu}+\left(\int_{\tau^{\prime}-1}^{\tau+1}\left(\int_{\Sigma_{t^{*}} \cap\left\{r \leq \frac{9}{10} t^{*}\right\}} G_{1}^{2}\right)^{1 / 2} d t^{*}\right)^{2}+\iint_{\mathscr{R}^{\prime}\left(\tau^{\prime}-1, \tau+1\right) \cap\left\{r \leq \frac{9}{10} t^{*}\right\}} G_{1}^{2}\right. \\
&\left.+\sum_{m=0}^{1} \iint_{\mathscr{R}\left(\tau^{\prime}-1, \tau+1\right) \cap\left\{r \leq \frac{9}{10} t^{*}\right\}} r^{1+\delta}\left(\partial_{t^{*}}^{m} G_{2}\right)^{2}+\sup _{t^{*} \in\left[\tau^{\prime}-1, \tau+1\right]} \int_{\Sigma_{t^{*}} \cap\left\{|r-3 M| \leq \frac{1}{8} M\right\}} G_{2}^{2}\right) .
\end{aligned}
$$

We can now conclude the proposition using Proposition 32.

We can remove the degeneracy around $r \sim 3 M$ using an extra derivative.

Proposition 34. Let $G=G_{1}+G_{2}$ be any way to decompose the function $G$. Then for $\tau^{\prime} \leq \tau \leq(1.1) \tau^{\prime}$, we have

(1) the localized boundedness estimate

$$
\begin{aligned}
& \iint_{\mathscr{R}\left(\tau^{\prime}, \tau\right) \cap\left\{r \leq \frac{1}{2} t^{*}\right\}} K^{X_{1}}(\Phi) \\
& \leq C\left(\sum_{m=0}^{1} \int_{\Sigma_{\tau^{\prime}}} J_{\mu}^{N}\left(\partial_{t^{*}}^{m} \Phi\right) n_{\Sigma_{\tau^{\prime}}}^{\mu}+\sum_{m=0}^{1}\left(\int_{\tau^{\prime}-1}^{\tau+1}\left(\int_{\Sigma_{t^{*}} \cap\left\{r \leq \frac{9}{10} t^{*}\right\}}\left(\partial_{t^{*}}^{m} G_{1}\right)^{2}\right)^{1 / 2} d t^{*}\right)^{2}\right. \\
& +\sum_{m=0}^{1} \iint_{\mathscr{R}\left(\tau^{\prime}-1, \tau+1\right) \cap\left\{r \leq \frac{9}{10} *^{*}\right\}}\left(\partial_{t^{*}}^{m} G_{1}\right)^{2}+\sum_{m=0}^{2} \iint_{\mathscr{R}\left(\tau^{\prime}-1, \tau+1\right) \cap\left\{r \leq \frac{9}{10} t^{*}\right\}} r^{1+\delta}\left(\partial_{t^{*}}^{m} G_{2}\right)^{2} \\
& \left.+\sup _{t^{*} \in\left[\tau^{\prime}-1, \tau+1\right]} \sum_{m=0}^{1} \int_{\Sigma_{t^{*}} \cap\left\{|r-3 M| \leq \frac{1}{8} M\right\} \cap\left\{r \leq \frac{9}{10} t^{*}\right\}}\left(\partial_{t^{*}}^{m} G_{2}\right)^{2}\right) ;
\end{aligned}
$$


(2) the localized decay estimate

$$
\begin{aligned}
& \iint_{\mathscr{R}\left(\tau^{\prime}, \tau\right) \cap\left\{r \leq \frac{1}{2} t^{*}\right\}} K^{X_{1}}(\Phi) \\
& \leq C\left(\tau^{-2} \sum_{m=0}^{1} \int_{\Sigma_{\tau^{\prime}}} J_{\mu}^{Z+N, w^{Z}}\left(\partial_{t^{*}}^{m} \Phi\right) n_{\Sigma_{\tau^{\prime}}}^{\mu}+C \sum_{m=0}^{1} \int_{\Sigma_{\tau^{\prime}} \cap\left\{r \leq r_{Y}^{-}\right\}} J_{\mu}^{N}\left(\partial_{t^{*}}^{m} \Phi\right) n_{\Sigma_{\tau^{\prime}}}^{\mu}\right) \\
& \quad+C\left(\sum_{m=0}^{1}\left(\int_{\tau^{\prime}-1}^{\tau+1}\left(\int_{\Sigma_{t^{*}} \cap\left\{r \leq \frac{9}{10} t^{*}\right\}}\left(\partial_{t^{*}}^{m} G_{1}\right)^{2}\right)^{1 / 2} d t^{*}\right)^{2}+\sum_{m=0}^{1} \iint_{\mathscr{R}\left(\tau^{\prime}-1, \tau+1\right) \cap\left\{r \leq \frac{9}{10} t^{*}\right\}}\left(\partial_{t^{*}}^{m} G_{1}\right)^{2}\right. \\
& \left.\quad+\sum_{m=0}^{2} \iint_{\mathscr{R}\left(\tau^{\prime}-1, \tau+1\right) \cap\left\{r \leq \frac{9}{10} t^{*}\right\}} r^{1+\delta}\left(\partial_{t^{*}}^{m} G_{2}\right)^{2}+\sup _{t^{*} \in\left[\tau^{\prime}-1, \tau+1\right]} \sum_{m=0} \int_{\Sigma_{t^{*}} \cap\left\{|r-3 M| \leq \frac{1}{8} M\right\} \cap\left\{r \leq \frac{9}{10} t^{*}\right\}}\left(\partial_{t^{*}}^{m} G_{2}\right)^{2}\right) .
\end{aligned}
$$

Proof. We repeat the argument in Proposition 33, using Proposition 29 instead of 28.

When using the conservation law for $Z$, we can ignore the part of the bulk term that has a good sign.

Definition 35. Let $K_{+}^{Z, w^{Z}}(\Phi)=\max \left\{K^{Z, w^{Z}}(\Phi), 0\right\}$.

Using the conservation law for the modified vector field, we have a one-sided bound:

\section{Proposition 36.}

$$
\begin{aligned}
& \int_{\Sigma_{\tau}} J_{\mu}^{Z, w^{Z}}(\Phi) n_{\Sigma_{\tau}}^{\mu}+\int_{\mathscr{H}\left(\tau^{\prime}, \tau\right)} J_{\mu}^{Z, w^{Z}}(\Phi) n_{\mathscr{H}^{+}}^{\mu} \\
& \leq C\left(\tau^{\prime}\right)^{2} \int_{\Sigma_{\tau^{\prime}} \cap\left\{r \leq r_{Y}^{-}\right\}} J_{\mu}^{N}(\Phi) n_{\Sigma_{\tau^{\prime}}}^{\mu}+\int_{\Sigma_{\tau^{\prime}}} J_{\mu}^{Z, w^{Z}}(\Phi) n_{\Sigma_{\tau^{\prime}}}^{\mu} \\
& \quad+\iint_{\mathscr{R}\left(\tau^{\prime}, \tau\right)} K_{+}^{Z, w^{Z}}(\Phi)+\left|\iint_{\mathscr{R}\left(\tau^{\prime}, \tau\right)}\left(u^{2} L \Phi+v^{2} \underline{L} \Phi-\frac{1}{4} w \Phi\right) G\right| .
\end{aligned}
$$

Remark. In the proposition, the left-hand side is not claimed to be positive. Note, however, that the right-hand side is positive by the remark on page 591 .

Remark. We note also that

$$
\int_{\mathscr{H}\left(\tau^{\prime}, \tau\right)} J_{\mu}^{Z, w^{Z}}(\Phi) n_{\mathscr{H}^{+}}^{\mu} \geq 0
$$

because $Z$ and $n_{\mathscr{H}^{+}}^{\mu}$ are both null and future directed and $w^{Z}=0$ on the event horizon.

To show that $\int_{\Sigma_{\tau}} J_{\mu}^{Z, w^{Z}}(\Phi) n_{\Sigma_{\tau}}^{\mu}$ is almost bounded, we will have to show that

$$
\int_{\Sigma_{\tau^{\prime}} \cap\left\{r \leq r_{Y}^{-}\right\}} J_{\mu}^{N}(\Phi) n_{\Sigma_{\tau^{\prime}}}^{\mu}
$$

in fact decays: 


\section{Proposition 37.}

$$
\begin{aligned}
& \int_{\Sigma_{\tau} \cap\left\{r \leq \frac{1}{2} t^{*}\right\}} J_{\mu}^{N}(\Phi) n_{\Sigma_{\tau}}^{\mu} \\
& \leq C^{2} \tau^{-2} \int_{\Sigma_{(1.1)^{-2} \tau} \cap\left\{r \leq r_{Y}^{-}\right\}} J_{\mu}^{N}(\Phi) n_{\Sigma_{(1.1)^{-2} \tau}}^{\mu}+C \tau^{-2} \int_{\Sigma_{(1.1)^{-2} \tau}} J_{\mu}^{Z, w^{Z}}(\Phi) n_{\Sigma_{(1.1)^{-1} \tau}^{\mu}}^{\mu} \\
& +C \tau^{-2} \iint_{\mathscr{R}\left((1.1)^{-2} \tau, \tau\right)} K_{+}^{Z, w^{Z}}(\Phi)+C \tau^{-2}\left|\iint_{\mathscr{R}\left((1.1)^{-2} \tau, \tau\right)}\left(u^{2} L \Phi+v^{2} \underline{L} \Phi-\frac{1}{4} w \Phi\right) G\right| \\
& +C\left(\left(\int_{(1.1)^{-2} \tau-1}^{\tau+1}\left(\int_{\Sigma_{t^{*} \cap\left\{r \leq \frac{9}{10} t^{*}\right\}}} G_{1}^{2}\right)^{1 / 2} d t^{*}\right)^{2}+\iint_{\mathscr{R}\left((1.1)^{-2} \tau-1, \tau+1\right) \cap\left\{r \leq \frac{9}{10} t^{*}\right\}} G_{1}^{2}\right) \\
& +C\left(\sum_{m=0}^{1} \iint_{\mathscr{R}\left((1.1)^{-2} \tau-1, \tau+1\right) \cap\left\{r \leq \frac{9}{10} t^{*}\right\}} r^{1+\delta}\left(\partial_{t^{*}}^{m} G_{2}\right)^{2}+\sup _{t^{*} \in\left[(1.1)^{-2} \tau-1, \tau+1\right]} \int_{\Sigma_{t^{*}} \cap\left\{|r-3 M| \leq \frac{1}{8} M\right\} \cap\left\{r \leq \frac{9}{10} t^{*}\right\}} G_{2}^{2}\right) .
\end{aligned}
$$

Proof. By Proposition 33(2) applied to the $t^{*}$ interval $\left[(1.1)^{-1} \tau, \tau\right]$, we have

$$
\begin{array}{r}
\iint_{\mathscr{R}\left((1.1)^{-1} \tau, \tau\right) \cap\left\{r \leq r_{Y}^{-}\right\}} K^{N}(\Phi) \leq C \tau^{-2} \int_{\Sigma_{(1.1)^{-1} \tau}} J_{\mu}^{Z+N, w^{Z}}(\Phi) n_{\Sigma_{(1.1)^{-1} \tau}^{\mu}}^{\mu}+C^{2} \int_{\Sigma_{(1.1)^{-1} \tau} \cap\left\{r \leq r_{Y}^{-}\right\}} J_{\mu}^{N}(\Phi) n_{\Sigma_{(1.1)^{-1} \tau}^{\mu}} \\
+C\left(\sum_{m=0}^{1} \iint_{\mathscr{R}^{\prime}\left((1.1)^{-1} \tau-1, \tau+1\right) \cap\left\{r \leq \frac{9}{10} t^{*}\right\}} r^{1+\delta}\left(\partial_{t^{*}}^{m} G\right)^{2}+\sup _{t^{*} \in\left[(1.1)^{-1} \tau-1, \tau+1\right]} \int_{\left.\Sigma_{t^{*} \cap\left\{\left\{|r-3 M| \leq \frac{1}{8} M\right\}\right.} G^{2}\right) .}\right.
\end{array}
$$

By taking the infimum there exists $\tilde{\tau} \in\left[(1.1)^{-1} \tau, \tau\right]$ such that

$$
\int_{\Sigma_{\tilde{\tau}} \cap\left\{r \leq r_{Y}^{-}\right\}} J_{\mu}^{N}(\Phi) n_{\Sigma_{\tilde{\tau}}}^{\mu} \leq C \tau^{-1} \iint_{\mathscr{R}^{(}\left((1.1)^{-1} \tau, \tau\right) \cap\left\{r \leq r_{Y}^{-}\right\}} K^{N}(\Phi) .
$$

Hence,

$$
\begin{aligned}
\int_{\Sigma_{\tilde{\tau}} \cap\left\{r \leq r_{Y}^{-}\right\}} & J_{\mu}^{N}(\Phi) n_{\Sigma_{\tilde{\tau}}}^{\mu} \\
\leq & C \tau^{-2} \int_{\Sigma_{(1.1)^{-1} \tau}} J_{\mu}^{Z+N, w^{Z}}(\Phi) n_{\Sigma_{(1.1)^{-1} \tau}}^{\mu}+C^{2} \tau^{-1} \int_{\Sigma_{(1.1)^{-1} \tau} \cap\left\{r \leq r_{Y}^{-}\right\}} J_{\mu}^{N}(\Phi) n_{\Sigma_{(1.1)^{-1} \tau}}^{\mu} \\
& +C \tau^{-1}\left(\sum_{m=0}^{1} \iint_{\mathscr{R}^{\prime}\left((1.1)^{-1} \tau-1, \tau+1\right) \cap\left\{r \leq \frac{9}{10} t^{*}\right\}} r^{1+\delta}\left(\partial_{t^{*}}^{m} G\right)^{2}+\sup _{t^{*} \in\left[(1.1)^{-1} \tau-1, \tau+1\right]} \int_{\Sigma_{t^{*}} \cap\left\{|r-3 M| \leq \frac{1}{8} M\right\}} G^{2}\right) .
\end{aligned}
$$

We apply Proposition 33(2) to the $t^{*}$ interval $[\tilde{\tau}, \tau]$ and use Proposition 28 and 36, getting 


$$
\begin{aligned}
& \int_{\Sigma_{\tau} \cap\left\{r \leq \frac{1}{2} t^{*}\right\}} J_{\mu}^{N}(\Phi) n_{\Sigma_{\tau}}^{\mu} \\
& \leq C\left(\tau^{-2} \int_{\Sigma_{\tilde{\tau}}} J_{\mu}^{Z+N, w^{Z}}(\Phi) n_{\Sigma_{\tilde{\tau}}}^{\mu}+C \int_{\Sigma_{\tilde{\tau}} \cap\left\{r \leq r_{Y}^{-}\right\}} J_{\mu}^{N}(\Phi) n_{\Sigma_{\tilde{\tau}}}^{\mu}\right) \\
& +C\left(\sum_{m=0}^{1} \iint_{\mathscr{R}\left((1.1)^{-1} \tau-1, \tau+1\right) \cap\left\{r \leq \frac{9}{10} t^{*}\right\}} r^{1+\delta}\left(\partial_{t^{*}}^{m} G\right)^{2}+\sup _{t^{*} \in\left[(1.1)^{-1} \tau-1, \tau+1\right]} \int_{\Sigma_{t^{*}} \cap\left\{|r-3 M| \leq \frac{1}{8} M\right\} \cap\left\{r \leq \frac{9}{10} t^{*}\right\}} G^{2}\right) \\
& \leq C \tau^{-2}\left(\int_{\Sigma_{\tilde{\tau}}} J_{\mu}^{Z+N, w^{Z}}(\Phi) n_{\Sigma_{\tilde{\tau}}}^{\mu}+\int_{\Sigma_{(1.1)-1}} J_{\mu}^{Z+N, w^{Z}}(\Phi) n_{\Sigma_{(1.1)-1}}^{\mu}\right) \\
& +C^{2} \tau^{-1} \int_{\Sigma_{(1.1)-1} \cap\left\{r \leq r_{Y}^{-}\right\}} J_{\mu}^{N}(\Phi) n_{\Sigma_{(1.1)^{-1} \tau}^{\mu}}^{\mu} \\
& +C\left(\sum_{m=0}^{1} \iint_{\mathscr{R}\left((1.1)^{-1} \tau-1, \tau+1\right) \cap\left\{r \leq \frac{9}{10} t^{*}\right\}} r^{1+\delta}\left(\partial_{t^{*}}^{m} G\right)^{2}+\sup _{t^{*} \in\left[(1.1)^{-1} \tau-1, \tau+1\right]} \int_{\Sigma_{t^{*}} \cap\left\{|r-3 M| \leq \frac{1}{8} M\right\} \cap\left\{r \leq \frac{9}{10} t^{*}\right\}} G^{2}\right) \\
& \leq C^{2} \tau^{-1} \int_{\Sigma_{(1.1)^{-1} \tau_{\tau}} \cap\left\{r \leq r_{Y}^{-}\right\}} J_{\mu}^{N}(\Phi) n_{\Sigma_{(1.1)^{-1} \tau}}^{\mu}+C \tau^{-2} \int_{\Sigma_{(1.1)^{-1} \tau}} J_{\mu}^{Z+N, w^{Z}}(\Phi) n_{\Sigma_{(1.1)^{-1} \tau}}^{\mu} \\
& +C \tau^{-2} \iint_{\mathscr{R}\left((1.1)^{-1} \tau, \tau\right)} K_{+}^{Z, w^{Z}}(\Phi)+C \tau^{-2}\left|\iint_{\mathscr{R}\left((1.1)^{-1} \tau, \tau\right)}\left(u^{2} \underline{L} \Phi+v^{2} L \Phi-\frac{1}{4} w \Phi\right) G\right|
\end{aligned}
$$

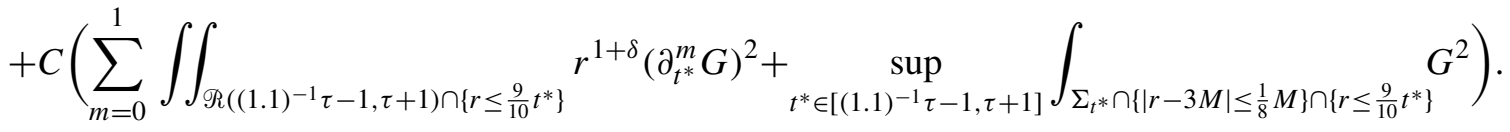

Replacing $\left[(1.1)^{-1} \tau, \tau\right]$ with $\left[(1.1)^{-2} \tau,(1.1)^{-1} \tau\right]$, we get also

$$
\begin{aligned}
& \int_{\Sigma_{(1.1)-1} \tau^{\cap}\left\{r \leq \frac{1}{2} t^{*}\right\}} J_{\mu}^{N}(\Phi) n_{\Sigma_{\tau}}^{\mu} \\
& \leq C^{2} \tau^{-1} \int_{\Sigma_{(1.1)-2} \cap\left\{r \leq r_{Y}^{-}\right\}} J_{\mu}^{N}(\Phi) n_{\Sigma_{(1.1)^{-2} \tau}}^{\mu}+C \tau^{-2} \int_{\Sigma_{(1.1)^{-2} \tau}} J_{\mu}^{Z+N, w^{Z}(\Phi) n_{\Sigma_{(1.1)-2}}^{\mu}} \\
&+C \tau^{-2} \iint_{\mathscr{R}\left((1.1)^{-2} \tau,(1.1)^{-1} \tau\right)} K_{+}^{Z, w^{Z}} C(\Phi)+C \tau^{-2}\left|\iint_{\mathscr{R}\left((1.1)^{-2} \tau,(1.1)^{-1} \tau\right)}\left(u^{2} L \Phi+v^{2} \underline{L} \Phi-\frac{1}{4} w \Phi\right) G\right| \\
&+C\left(\sum_{m=0}^{1} \iint_{\mathscr{R}\left((1.1)^{-2} \tau-1,(1.1)^{-1} \tau+1\right) \cap\left\{r \leq \frac{9}{10} t^{*}\right\}} r^{1+\delta}\left(\partial_{t^{*}}^{m} G\right)^{2}\right. \\
&\left.+\operatorname{sip}_{t^{*} \in\left[(1.1)^{-2} \tau-1,(1.1)^{-1} \tau+1\right]} \int_{\Sigma_{t^{*}} \cap\left\{|r-3 M| \leq \frac{1}{8} M\right\} \cap\left\{r \leq \frac{9}{10} t^{*}\right\}} G^{2}\right)
\end{aligned}
$$

Therefore, plugging this result into the previous, we get 


$$
\begin{aligned}
& \int_{\Sigma_{\tau} \cap\left\{r \leq \frac{1}{2} t^{*}\right\}} J_{\mu}^{N}(\Phi) n_{\Sigma_{\tau}}^{\mu} \\
& \leq C^{2} \tau^{-2} \int_{\Sigma_{(1.1)^{-2} \tau} \cap\left\{r \leq r_{Y}^{-}\right\}} J_{\mu}^{N}(\Phi) n_{\Sigma_{(1.1)^{-2} \tau}}^{\mu}+C \tau^{-2} \int_{\Sigma_{(1.1)^{-2} \tau}} J_{\mu}^{Z, w^{Z}}(\Phi) n_{\Sigma_{(1.1)^{-1} \tau}}^{\mu} \\
& \quad+C \tau^{-2} \iint_{\mathscr{R}\left((1.1)^{-2} \tau, \tau\right)} K_{+}^{Z, w^{Z}}(\Phi)+C \tau^{-2}\left|\iint_{\mathscr{R}\left((1.1)^{-2} \tau, \tau\right)}\left(u^{2} L \Phi+v^{2} \underline{L} \Phi-\frac{1}{4} w \Phi\right) G\right| \\
& \quad+C\left(\sum_{m=0}^{1} \iint_{\mathscr{R}\left((1.1)^{-2} \tau-1, \tau+1\right) \cap\left\{r \leq \frac{9}{10} t^{*}\right\}} r^{1+\delta}\left(\partial_{t^{*}}^{m} G\right)^{2}\right. \\
& \left.\quad+\sup _{t^{*} \in\left[(1.1)^{-2} \tau-1, \tau+1\right]} \int_{\Sigma_{t^{*}} \cap\left\{|r-3 M| \leq \frac{1}{8} M\right\} \cap\left\{r \leq \frac{9}{10} t^{*}\right\}} G^{2}\right) .
\end{aligned}
$$

Proposition 37 immediately gives control over the nondegenerate energy and conformal energy using Propositions 31 and 36, respectively:

Corollary 38. For any $\gamma<1$,

$$
\begin{aligned}
& \int_{\Sigma_{\tau}} J_{\mu}^{Z, w^{Z}}(\Phi) n_{\Sigma_{\tau}}^{\mu}+C \tau^{2} \int_{\Sigma_{\tau} \cap\{r \leq \gamma \tau\}} J_{\mu}^{N}(\Phi) n_{\Sigma_{\tau}}^{\mu} \\
& \leq C\left(\int_{\Sigma_{\tau_{0}}} J_{\mu}^{Z, w^{Z}}(\Phi) n_{\Sigma_{\tau_{0}}}^{\mu}+C \int_{\Sigma_{\tau_{0}}} J_{\mu}^{N}(\Phi) n_{\Sigma_{\tau_{0}}}^{\mu}\right. \\
& \left.+\iint_{\mathscr{R}\left(\tau_{0}, \tau\right)} K_{+}^{Z, w^{Z}}(\Phi)+\left|\iint_{\mathscr{R}\left(\tau_{0}, \tau\right)}\left(u^{2} \underline{L} \Phi+v^{2} L \Phi-\frac{1}{4} w \Phi\right) G\right|\right)
\end{aligned}
$$

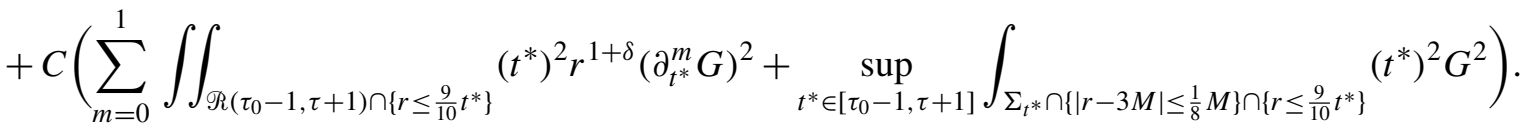

Proof. By Proposition 31,

$$
\tau^{2} \int_{\Sigma_{\tau} \cap\{r \leq \gamma \tau\}} J_{\mu}^{N}(\Phi) n_{\Sigma_{\tau}}^{\mu} \leq C \int_{\Sigma_{\tau}} J_{\mu}^{Z+N, w^{Z}}(\Phi) n_{\Sigma_{\tau}}^{\mu}+C^{2} \tau^{2} \int_{\Sigma_{\tau} \cap\left\{r \leq r_{Y}^{-}\right\}} J_{\mu}^{N}(\Phi) n_{\Sigma_{\tau}}^{\mu} .
$$

Therefore, by Propositions 36 and 37,

$$
\begin{aligned}
& \int_{\Sigma_{\tau}} J_{\mu}^{Z, w^{Z}}(\Phi) n_{\Sigma_{\tau}}^{\mu}+C \tau^{2} \int_{\Sigma_{\tau} \cap\{r \leq \gamma \tau\}} J_{\mu}^{N}(\Phi) n_{\Sigma_{\tau}}^{\mu} \\
& \leq C\left(\int_{\Sigma_{(1.1)^{-2} \tau}} J_{\mu}^{N}(\Phi) n_{\Sigma_{(1.1)^{-2} \tau}^{\mu}}^{\mu}+\int_{\Sigma_{(1.1)^{-2} \tau}} J_{\mu}^{Z, w^{Z}}(\Phi) n_{\Sigma_{(1.1)^{-2} \tau}}^{\mu}+\iint_{\mathscr{R}^{(}\left((1.1)^{-2} \tau, \tau\right)} K_{+}^{Z, w^{Z}}(\Phi)\right. \\
& \left.\quad+\left|\iint_{\mathscr{R}\left((1.1)^{-2} \tau, \tau\right)}\left(u^{2} \underline{L} \Phi+v^{2} L \Phi-\frac{1}{4} w \Phi\right) G\right|\right) \\
& \quad+C \tau^{2}\left(\sum_{m=0}^{1} \iint_{\mathscr{R}\left((1.1)^{-2} \tau-1, \tau+1\right)} r^{1+\delta}\left(\partial_{t^{*}}^{m} G\right)^{2}+\sup _{t^{*} \in\left[(1.1)^{-2} \tau-1, \tau+1\right]} \int_{\Sigma_{t^{*}} \cap\left\{|r-3 M| \leq \frac{1}{8} M\right\} \cap\left\{r \leq \frac{9}{10} t^{*}\right\}} G^{2}\right) .
\end{aligned}
$$

We then use the same estimate for $\left[(1.1)^{-4} \tau,(1.1)^{-2} \tau\right],\left[(1.1)^{-6} \tau,(1.1)^{-4} \tau\right], \ldots$ 
The term $\iint_{\mathscr{R}\left(\tau_{0}, \tau\right)} K_{+}^{Z, w^{Z}}(\Phi)$ can be controlled. Here is where the control of the logarithmic divergences from the red-shift vector field is crucially used.

Proposition 39. We have

$$
\iint_{\mathscr{R}\left(\tau^{\prime}, \tau\right)} K_{+}^{Z, w^{Z}}(\Phi) \leq C \iint_{\mathscr{R}\left(\tau^{\prime}, \tau\right) \cap\left\{r \geq r_{Y}^{-}\right\}} t^{*}\left(r^{-2} J_{\mu}^{N}(\Phi) n_{\Sigma_{\bar{\tau}}}^{\mu}+r^{-4} \Phi\right)+\epsilon \iint_{\mathscr{R}\left(\tau^{\prime}, \tau\right) \cap\left\{r \leq r_{Y}^{-}\right\}}\left(t^{*}\right)^{2} K^{N}(\Phi) .
$$

Proof. See [Dafermos and Rodnianski 2008].

The bulk term arising from the inhomogeneous term $G$ can also be controlled.

\section{Proposition 40.}

$$
\begin{aligned}
& \left|\iint_{\mathscr{R}\left(\tau_{0}, \tau\right)}\left(u^{2} \underline{L} \Phi+v^{2} L \Phi-\frac{1}{4} w \Phi\right) G\right| \\
& \leq \delta^{\prime} \iint_{\mathscr{R}\left(\tau_{0}, \tau\right) \cap\left\{r \leq \frac{1}{2} t^{*}\right\}}\left(t^{*}\right)^{2} K^{X_{0}}(\Phi)+\delta^{\prime} \iint_{\mathscr{R}\left(\tau_{0}, \tau\right) \cap\left\{r \leq r_{Y}^{-}\right\}}\left(t^{*}\right)^{2} K^{N}(\Phi) \\
& +\delta^{\prime} \sup _{t^{*} \in\left[\tau_{0}, \tau\right]}\left(\int_{\Sigma_{t^{*}} \cap\left\{r \geq \frac{1}{2} t^{*}\right\}} J_{\mu}^{Z+N, w^{Z}}(\Phi) n_{\Sigma_{t^{*}}}^{\mu}+\left(t^{*}\right)^{2} \int_{\Sigma_{t^{*} \cap\left\{r \leq \frac{23}{8} M\right\}}} J_{\mu}^{N}(\Phi) n_{\Sigma_{t^{*}}}^{\mu}\right) \\
& +C\left(\delta^{\prime}\right)^{-1} \sum_{m=0}^{1} \iint_{\mathscr{R}\left(\tau_{0}, \tau\right) \cap\left\{r \leq \frac{1}{2} t^{*}\right\}}\left(t^{*}\right)^{2} r^{1+\delta}\left(\partial_{t^{*}}^{m} G\right)^{2}+C\left(\delta^{\prime}\right)^{-1}\left(\int_{\tau_{0}}^{\tau}\left(\int_{\Sigma_{t^{*}} \cap\left\{r \geq \frac{1}{2} t^{*}\right\}} r^{2} G^{2}\right)^{1 / 2} d t^{*}\right)^{2} \\
& +C\left(\delta^{\prime}\right)^{-1} \sup _{t^{*} \in\left[\tau_{0}, \tau\right]} \int_{\Sigma_{t^{*}} \cap\left\{r_{Y}^{-} \leq r \leq \frac{25}{8} M\right\}}\left(t^{*}\right)^{2} G^{2} .
\end{aligned}
$$

Proof. Two regions require particular care. The first is the region $\left\{r \leq r_{Y}^{-}\right\}$, since the coefficients of the vector field $Z$ are not bounded as $r \rightarrow r_{+}$. The other is the region $\left\{|r-3 M| \leq \frac{1}{8} M\right\}$. This is where trapping occurs and where the integrated decay estimate degenerates or loses derivatives. We first look at the region $\left\{r \leq r_{+}\right\}$using the null frame:

$$
\begin{aligned}
\iint_{\mathscr{R}\left(\tau_{0}, \tau\right) \cap\left\{r \leq r_{Y}^{-}\right\}}\left(u^{2} \underline{L} \Phi+v^{2} L \Phi-\frac{1}{4} w \Phi\right) G & \\
\leq & C \iint_{\mathscr{R}\left(\tau_{0}, \tau\right) \cap\left\{r \leq r_{Y}^{-}\right\}}\left(\left(t^{*}\right)^{2}+\left(r_{S}^{*}\right)^{2}\right)\left(\left|\nabla_{\hat{V}} \Phi G\right|+(1-\mu)\left|\nabla_{\hat{Y}} \Phi G\right|+(1-\mu) \sum_{A}\left|\nabla_{E_{A}} \Phi G\right|\right) \\
& \text { (using Proposition 30) } \\
\leq & C \iint_{\mathscr{R}\left(\tau_{0}, \tau\right) \cap\left\{r \leq r_{Y}^{-}\right\}}\left(t^{*}\right)^{2}\left(|\log | r-r_{+}||^{2}\left|\nabla_{\hat{V}} \Phi G\right|+\left|\nabla_{\hat{Y}} \Phi G\right|+\sum_{A}\left|\nabla_{E_{A}} \Phi G\right|\right) \\
\leq & \delta^{\prime} \iint_{\mathscr{R}\left(\tau_{0}, \tau\right) \cap\left\{r \leq r_{Y}^{-}\right\}}\left(t^{*}\right)^{2}\left(|\log | r-r_{+} \|^{4}\left(\nabla_{\hat{V}} \Phi\right)^{2}+\left(\nabla_{\hat{Y}} \Phi\right)^{2}+\sum_{A}\left(\nabla_{E_{A}} \Phi\right)^{2}\right) \\
& \\
\leq & \delta^{\prime} \iint_{\mathscr{R}\left(\tau_{0}, \tau\right) \cap\left\{r \leq r_{Y}^{-}\right\}}\left(t^{*}\right)^{2} K^{N}(\Phi)+C\left(\delta^{\prime}\right)^{-1} \iint_{\mathscr{R}\left(\tau_{0}, \tau\right) \cap\left\{r \leq r_{Y}^{-}\right\}}\left(t^{*}\right)^{2} G^{2}
\end{aligned}
$$


For the region $\left\{r_{Y}^{-} \leq r \leq \frac{25}{8} M\right\}$, where trapping occurs, we integrate by parts in $t^{*}$ so that the bulk term does not have $\partial_{t^{*}} \Phi$, which cannot be controlled by the integrated decay estimate.

$$
\begin{gathered}
\left|\iint_{\mathscr{R}\left(\tau_{0}, \tau\right) \cap\left\{r_{Y}^{-} \leq r \leq \frac{25}{8} M\right\}}\left(u^{2} \underline{L} \Phi+v^{2} L \Phi-\frac{1}{4} w \Phi\right) G\right| \\
\leq C \iint_{\mathscr{R}\left(\tau_{0}, \tau\right) \cap\left\{r_{Y}^{-} \leq r \leq \frac{25}{8} M\right\}}\left(t^{*}\right)^{2}\left|\partial_{r} \Phi G\right|+\left(t^{*}\right)^{2}\left|\Phi \partial_{t^{*}} G\right|+t^{*}|\Phi G|+\int_{\Sigma_{\tau}} \tau^{2}|\Phi G|+\int_{\Sigma_{\tau_{0}}} \tau_{0}^{2}|\Phi G| \\
\leq C\left(\iint_{\mathscr{R}\left(\tau_{0}, \tau\right) \cap\left\{r \leq \frac{25}{8} M\right\}}\left(t^{*}\right)^{2}\left(\Phi^{2}+\left(\partial_{r} \Phi\right)^{2}\right)\right)^{1 / 2}\left(\sum_{m=0}^{1} \iint_{\mathscr{R}\left(\tau_{0}, \tau\right) \cap\left\{r_{Y}^{-} \leq r \leq \frac{25}{8} M\right\}}\left(t^{*}\right)^{2}\left(\partial_{t^{*}}^{m} G\right)^{2}\right)^{1 / 2} \\
+\delta^{\prime} \int_{\Sigma_{\tau} \cap\left\{r_{Y}^{-} \leq r \leq r_{Y}^{-} \leq \frac{25}{8} M\right\}} \tau^{2} J_{\mu}^{N}(\Phi) n_{\Sigma_{\tau}}^{\mu}+\delta^{\prime} \int_{\Sigma_{\tau_{0} \cap\left\{r_{Y}^{-} \leq r \leq \frac{25}{8} M\right\}} \tau_{0}^{2} J_{\mu}^{N}(\Phi) n_{\Sigma_{\tau_{0}}}^{\mu}}+C\left(\delta^{\prime}\right)^{-1} \sup _{t^{*} \in\left[\tau_{0}, \tau\right]} \int_{\Sigma_{t^{*} \cap\left\{r_{Y}^{-} \leq r \leq \frac{25}{8} M\right\}}\left(t^{*}\right)^{2} G^{2}}
\end{gathered}
$$

using Proposition 21. We then move to the region $\left\{\frac{25}{8} M \leq r \leq \frac{1}{2} t^{*}\right\}$ :

$$
\begin{aligned}
& \left|\iint_{\mathscr{R}\left(\tau_{0}, \tau\right) \cap\left\{\frac{25}{8} M \leq r \leq \frac{1}{2} t^{*}\right\}}\left(u^{2} \underline{L} \Phi+v^{2} L \Phi-\frac{1}{4} w \Phi\right) G\right| \\
& \quad \leq C \iint_{\mathscr{R}\left(\tau_{0}, \tau\right) \cap\left\{\frac{25}{8} M \leq r \leq \frac{1}{2} t^{*}\right\}}\left(\left(t^{*}\right)^{2}|\partial \Phi|+t^{*}|\Phi|\right)|G| \\
& \quad \leq C\left(\iint_{\mathscr{R}\left(\tau_{0}, \tau\right) \cap\left\{\frac{25}{8} M \leq r \leq \frac{1}{2} t^{*}\right\}}\left(t^{*}\right)^{2}\left(r^{-3-\delta} \Phi^{2}+r^{-1-\delta} J_{\mu}^{N}(\Phi) n_{\Sigma_{t^{*}}}^{\mu}\right)\right)^{1 / 2} \\
& \quad \times\left(\iint_{\mathscr{R}\left(\tau_{0}, \tau\right) \cap\left\{\frac{25}{8} M \leq r \leq \frac{1}{2} t^{*}\right\}}\left(r^{3+\delta}+\left(t^{*}\right)^{2} r^{1+\delta}\right) G^{2}\right)^{1 / 2} \\
& \quad \leq C\left(\iint_{\mathscr{R}\left(\tau_{0}, \tau\right) \cap\left\{\frac{25}{8} M \leq r \leq \frac{1}{2} t^{*}\right\}}\left(t^{*}\right)^{2}\left(r^{-3-\delta} \Phi^{2}+r^{-1-\delta} J_{\mu}^{N}(\Phi) n_{\Sigma_{t^{*}}}^{\mu}\right)\right)^{1 / 2} \times\left(\iint_{\mathscr{R}\left(\tau_{0}, \tau\right) \cap\left\{\frac{25}{8} M \leq r \leq \frac{1}{2} t^{*}\right\}}\left(t^{*}\right)^{2} r^{1+\delta} G^{2}\right)^{1 / 2}
\end{aligned}
$$

Finally, we estimate in the region $\left\{r \geq \frac{1}{2} t^{*}\right\}$ :

$$
\begin{aligned}
\left|\iint_{\mathscr{R}\left(\tau_{0}, \tau\right) \cap\left\{r \geq \frac{1}{2} t^{*}\right\}}\left(u^{2} \underline{L} \Phi+v^{2} L \Phi-\frac{1}{4} w \Phi\right) G\right| \\
\leq C \sup _{t^{*} \in\left[\tau_{0}, \tau\right]}\left(\int_{\Sigma_{t^{*}} \cap\left\{r \geq \frac{1}{2} t^{*}\right\}} J_{\mu}^{Z, w^{Z}}(\Phi) n_{\Sigma_{t^{*}}}^{\mu}+\left(t^{*}\right)^{2} \int_{\Sigma_{t^{*} \cap\left\{r \leq r_{Y}^{-}\right\}}} J_{\mu}^{N}(\Phi) n_{\Sigma_{t^{*}}}^{\mu}\right)^{1 / 2} \\
\times \int_{\tau_{0}}^{\tau}\left(\int_{\left.\Sigma_{t^{*} \cap\left\{r \geq \frac{1}{2} t^{*}\right\}} r^{2} G^{2}\right)^{1 / 2} d t^{*},}\right.
\end{aligned}
$$

where we have used Proposition 31. The proposition follows from Cauchy-Schwarz.

We have therefore proved the following decay result associated to the vector field $Z$. 
Proposition 41. For sufficiently small positive $\delta$ and $\delta^{\prime}$ and $0 \leq \gamma<1$, there exist $c=c(\delta, \gamma)$ and $C=C(\delta, \gamma)$ such that the following estimate holds for any solution to $\square_{g_{K}} \Phi=G$ :

$$
\begin{aligned}
& c \int_{\Sigma_{\tau}} J_{\mu}^{Z, w^{Z}}(\Phi) n_{\Sigma_{\tau}}^{\mu}+\tau^{2} \int_{\Sigma_{\tau} \cap\{r \leq \gamma \tau\}} J_{\mu}^{N}(\Phi) n_{\Sigma_{\tau}}^{\mu} \\
& \leq C \int_{\Sigma_{\tau_{0}}} J_{\mu}^{Z+C N, w^{Z}}(\Phi) n_{\Sigma_{\tau_{0}}}^{\mu}+C \iint_{\mathscr{R}\left(\tau_{0}, \tau\right)} t^{*} r^{-1+\delta} K^{X_{1}}(\Phi) \\
& \quad+C \delta^{\prime} \iint_{\mathscr{R}\left(\tau_{0}, \tau\right) \cap\left\{r \leq \frac{1}{2} t^{*}\right\}}\left(t^{*}\right)^{2} K^{X_{0}}(\Phi)+C\left(\delta^{\prime}+\epsilon\right) \iint_{\mathscr{R}\left(\tau_{0}, \tau\right) \cap\left\{r \leq r_{Y}^{-}\right\}}\left(t^{*}\right)^{2} K^{N}(\Phi) \\
& \quad+C\left(\delta^{\prime}\right)^{-1}\left(\int_{\tau_{0}}^{\tau}\left(\int_{\Sigma_{t^{*}} \cap\left\{r \geq \frac{1}{2} t^{*}\right\}} r^{2} G^{2}\right)^{1 / 2} d t^{*}\right)^{2}+C\left(\delta^{\prime}\right)^{-1} \sum_{m=0} \iint_{\mathscr{R}\left(\tau_{0}, \tau\right) \cap\left\{r \leq \frac{9}{10} t^{*}\right\}}\left(t^{*}\right)^{2} r^{1+\delta}\left(\partial_{t^{*}}^{m} G\right)^{2} \\
& \quad+C\left(\delta^{\prime}\right)^{-1} \sup _{t^{*} \in\left[\tau_{0}, \tau\right]} \int_{\Sigma_{t^{*} \cap\left\{r_{Y}^{-} \leq r \leq \frac{25}{8} M\right\}}\left(t^{*}\right)^{2} G^{2} .}
\end{aligned}
$$

\section{Estimates for solutions to $\square_{g_{K}} \Phi=0$}

From this point on, we consider $\square_{g_{K}} \Phi=0$. In this section, we write down the energy estimates derived by Dafermos and Rodnianski [2008]. These will be used in later sections.

\section{Proposition 42.}

$$
\begin{aligned}
\tau^{2} \int_{\Sigma_{\tau} \cap\left\{r \leq \frac{1}{2} \tau\right\}} J_{\mu}^{N}(\Phi) n_{\Sigma_{\tau}}^{\mu}+c \int_{\Sigma_{\tau}} J_{\mu}^{Z+N, w^{Z}}(\Phi) n_{\Sigma_{\tau}}^{\mu} & \\
& \leq C \tau^{\eta} \sum_{m=0}^{2}\left(\int_{\Sigma_{\tau_{0}}} J_{\mu}^{Z, w^{Z}}\left(\partial_{t^{*}}^{m} \Phi\right) n_{\Sigma_{\tau_{0}}}^{\mu}+\int_{\Sigma_{\tau_{0}}} J_{\mu}^{N}\left(\partial_{t^{*}}^{m} \Phi\right) n_{\Sigma_{\tau_{0}}}^{\mu}\right)
\end{aligned}
$$

Proof. We introduce the bootstrap assumptions:

$$
\begin{gathered}
\tau^{2} \int_{\Sigma_{\tau} \cap\left\{r \leq \frac{1}{2} \tau\right\}} J_{\mu}^{N}(\Phi) n_{\Sigma_{\tau}}^{\mu}+c \int_{\Sigma_{\tau}} J_{\mu}^{Z+N, w^{Z}}(\Phi) n_{\Sigma_{\tau}}^{\mu} \leq A^{2} \tau^{\eta} \sum_{m=0}^{2} \int_{\Sigma_{\tau_{0}}} J_{\mu}^{Z+C N, w^{Z}}\left(\partial_{t^{*}}^{m} \Phi\right) n_{\Sigma_{\tau_{0}}}^{\mu}, \\
\tau^{2} \int_{\Sigma_{\tau} \cap\left\{r \leq \frac{1}{2} \tau\right\}} J_{\mu}^{N}\left(\partial_{t^{*}} \Phi\right) n_{\Sigma_{\tau}}^{\mu}+c \int_{\Sigma_{\tau}} J_{\mu}^{Z+N, w^{Z}}(\Phi) n_{\Sigma_{\tau}}^{\mu} \leq A \tau^{1+\eta} \sum_{m=0}^{2} \int_{\Sigma_{\tau_{0}}} J_{\mu}^{Z+C N, w^{Z}}\left(\partial_{t^{*}}^{m} \Phi\right) n_{\Sigma_{\tau_{0}}}^{\mu} .
\end{gathered}
$$

Here we think of $\eta$ as a small positive number. We divide the interval $\left[\tau_{0}, \tau\right]$ dyadically into $\tau_{0} \leq \tau_{1} \leq$ $\cdots \leq \tau_{n-1} \leq \tau_{n}=\tau$ with $\tau_{i+1} \leq(1.1) \tau_{i}$ and $n$ the smallest integer for doing such division. We then have $n \sim \log \left|\tau-\tau^{\prime}\right|$. We can now apply Proposition 33 on the intervals $\left[\tau_{i-1}, \tau_{i}\right]$ and use the bootstrap 
assumption (16):

$$
\begin{aligned}
\iint_{\mathscr{R}\left(\tau_{i-1}, \tau_{i}\right) \cap\left\{r \leq \frac{1}{2} t^{*}\right\}} K^{X_{0}}(\Phi) & +\iint_{\mathscr{R}\left(\tau_{i-1}, \tau_{i}\right) \cap\left\{r \leq r_{Y}^{-}\right\}} K^{N}(\Phi) \\
\leq & C\left(\tau_{i}^{-2} \int_{\Sigma_{\tau_{i-1}}} J_{\mu}^{Z, w^{Z}}(\Phi) n_{\Sigma_{\tau_{i-1}}^{\mu}}^{\mu}+C \int_{\Sigma_{\tau_{i-1}} \cap\left\{r \leq r_{Y}^{-}\right\}} J_{\mu}^{N}(\Phi) n_{\Sigma_{\tau_{i-1}}}^{\mu}\right) \\
\leq & C A^{2} \tau_{i}^{-2+\eta} \sum_{m=0}^{2} \int_{\Sigma_{\tau_{0}}} J_{\mu}^{Z+C N, w^{Z}}\left(\partial_{t^{*}}^{m} \Phi\right) n_{\Sigma_{\tau_{0}}}^{\mu} .
\end{aligned}
$$

Similarly, we can apply Proposition 33 on the intervals $\left[\tau_{i-1}, \tau_{i}\right]$ for $\partial_{t^{*}} \Phi$ and use the bootstrap assumption (17):

$$
\begin{aligned}
\iint_{\mathscr{R}\left(\tau_{i-1}, \tau_{i}\right) \cap\left\{r \leq \frac{1}{2} t^{*}\right\}} K^{X_{0}}\left(\partial_{t^{*}} \Phi\right) & +\iint_{\mathscr{R}\left(\tau_{i-1}, \tau_{i}\right) \cap\left\{r \leq r_{Y}^{-}\right\}} K^{N}\left(\partial_{t^{*}} \Phi\right) \\
& \leq C\left(\tau_{i}^{-2} \int_{\Sigma_{\tau_{i-1}}} J_{\mu}^{Z, w^{Z}}\left(\partial_{t^{*}} \Phi\right) n_{\Sigma_{\tau_{i-1}}}^{\mu}+C \int_{\Sigma_{\tau_{i-1}} \cap\left\{r \leq r_{Y}^{-}\right\}} J_{\mu}^{N}\left(\partial_{t^{*}} \Phi\right) n_{\Sigma_{\tau_{i-1}}}^{\mu}\right) \\
& \leq C A \tau_{i}^{-1+\eta} \sum_{m=0}^{2} \int_{\Sigma_{\tau_{0}}} J_{\mu}^{Z+C N, w^{Z}}\left(\partial_{t^{*}}^{m} \Phi\right) n_{\Sigma_{\tau_{0}}}^{\mu} .
\end{aligned}
$$

By Proposition 29, we have

$$
\iint_{\Re\left(\tau_{i-1}, \tau_{i}\right)} r^{-1+\delta} K^{X_{1}}\left(\partial_{t^{*}} \Phi\right) \leq C \sum_{m=0}^{1} \int_{\Sigma_{\tau_{0}}} J_{\mu}^{N}\left(\partial_{t^{*}}^{m} \Phi\right) n_{\Sigma_{\tau_{0}}}^{\mu} .
$$

By Propositions 29 and 34, we have

$$
\begin{aligned}
\iint_{\mathscr{R}\left(\tau_{i-1}, \tau_{i}\right)} r^{-1+\delta} K^{X_{1}}(\Phi) & \leq C \iint_{\mathscr{R}\left(\tau_{i-1}, \tau_{i}\right) \cap\left\{r \leq \frac{1}{2} t^{*}\right\}} K^{X_{1}}(\Phi)+C \tau_{i}^{-1+\delta} \iint_{\mathscr{R}\left(\tau_{i-1}, \tau_{i}\right) \cap\left\{r \geq \frac{1}{2} t^{*}\right\}} K^{X_{1}}(\Phi) \\
& \leq C A \tau_{i}^{-1+\eta} \sum_{m=0}^{2} \int_{\Sigma_{\tau_{0}}} J_{\mu}^{Z+C N, w^{Z}}\left(\partial_{t^{*}}^{m} \Phi\right) n_{\Sigma_{\tau_{0}}}^{\mu} .
\end{aligned}
$$

Applying Proposition 41, we get

$$
\begin{gathered}
c \int_{\Sigma_{\tau}} J_{\mu}^{Z+N, w^{Z}}(\Phi) n_{\Sigma_{\tau}}^{\mu}+\tau^{2} \int_{\Sigma_{\tau} \cap\{r \leq \gamma \tau\}} J_{\mu}^{N}(\Phi) n_{\Sigma_{\tau}}^{\mu} \\
\leq C \int_{\Sigma_{\tau_{0}}} J_{\mu}^{Z+C N, w^{Z}}(\Phi) n_{\Sigma_{\tau_{0}}}^{\mu}+C \iint_{\mathscr{R}\left(\tau_{0}, \tau\right)} t^{*} r^{-1+\delta} K^{X_{1}}(\Phi) \\
\quad+C \delta^{\prime} \iint_{\mathscr{R}\left(\tau_{0}, \tau\right) \cap\left\{r \leq \frac{1}{2} t^{*}\right\}}\left(t^{*}\right)^{2} K^{X_{0}}(\Phi)+C\left(\delta^{\prime}+\epsilon\right) \iint_{\mathscr{R}\left(\tau_{0}, \tau\right) \cap\left\{r \leq r_{Y}^{-}\right\}}\left(t^{*}\right)^{2} K^{N}(\Phi) \\
\leq\left(C+\left(C+C A+C A^{2}\left(2 \delta^{\prime}+\epsilon\right)\right) \sum_{i=0}^{n-1} \tau_{i}^{\eta}\right) \sum_{m=0}^{2} \int_{\Sigma_{\tau_{0}}} J_{\mu}^{Z+C N, w^{Z}}\left(\partial_{t^{*}}^{m} \Phi\right) n_{\Sigma_{\tau_{0}}}^{\mu}
\end{gathered}
$$




$$
\leq\left(C+\eta^{-1}\left(C+C A+C A^{2}\left(2 \delta^{\prime}+\epsilon\right)\right) \tau^{\eta}\right) \sum_{m=0}^{2} \int_{\Sigma_{\tau_{0}}} J_{\mu}^{Z+C N, w^{Z}}\left(\partial_{t^{*}}^{m} \Phi\right) n_{\Sigma_{\tau_{0}}}^{\mu} .
$$

Now take $A$ large, $\epsilon=\eta /(4 C)$ and $\delta^{\prime}=\epsilon / 2$, we improve (16). Applying Proposition 41 again, this time to $\partial_{t^{*}} \Phi$, we have

$$
\begin{gathered}
c \int_{\Sigma_{\tau}} J_{\mu}^{Z, w^{Z}}\left(\partial_{t^{*}} \Phi\right) n_{\Sigma_{\tau}}^{\mu}+\tau^{2} \int_{\Sigma_{\tau} \cap\{r \leq \gamma \tau\}} J_{\mu}^{N}\left(\partial_{t^{*}} \Phi\right) n_{\Sigma_{\tau}}^{\mu} \\
\leq C \int_{\Sigma_{\tau_{0}}} J_{\mu}^{Z+C N, w^{Z}}\left(\partial_{t^{*}} \Phi\right) n_{\Sigma_{\tau_{0}}}^{\mu}+C \iint_{\mathscr{R}\left(\tau_{0}, \tau\right)} t^{*} r^{-1+\delta} K^{X_{1}}\left(\partial_{t^{*}} \Phi\right) \\
\quad+C \delta^{\prime} \iint_{\mathscr{R}\left(\tau_{0}, \tau\right) \cap\left\{r \leq \frac{1}{2} t^{*}\right\}}\left(t^{*}\right)^{2} K^{X_{0}}\left(\partial_{t^{*}} \Phi\right)+C\left(\delta^{\prime}+\epsilon\right) \iint_{\mathscr{R}\left(\tau_{0}, \tau\right) \cap\left\{r \leq r_{Y}^{-}\right\}}\left(t^{*}\right)^{2} K^{N}\left(\partial_{t^{*}} \Phi\right) \\
\leq\left(C+C \sum_{i=0}^{n-1} \tau_{i}+C A\left(2 \delta^{\prime}+\epsilon\right) \sum_{i=0}^{n-1} \tau_{i}^{1+\eta}\right) \sum_{m=0}^{2} \int_{\Sigma_{\tau_{0}}} J_{\mu}^{Z+C N, w^{Z}}\left(\partial_{t^{*}}^{m} \Phi\right) n_{\Sigma_{\tau_{0}}}^{\mu} \\
\leq\left(C+C \tau+C A\left(2 \delta^{\prime}+\epsilon\right) \tau^{1+\eta}\right) \sum_{m=0}^{2} \int_{\Sigma_{\tau_{0}}} J_{\mu}^{Z+C N, w^{Z}}\left(\partial_{t^{*}}^{m} \Phi\right) n_{\Sigma_{\tau_{0}}}^{\mu}
\end{gathered}
$$

Now taking $A$ large, $\delta^{\prime}=\epsilon$ and $\epsilon$ sufficiently small, we also improve (17).

In particular, the theorem of Dafermos and Rodnianski [2008] is retrieved.

Corollary 43 (Dafermos and Rodnianski). Suppose $\square_{g_{K}} \Phi=0$. Then for all $\eta>0$ and all $M>0$ there exists $a_{0}$ such that the following estimates hold on Kerr spacetimes with $(M, a)$ for which $a \leq a_{0}$ :

(1) The boundedness of nondegenerate energy:

$\int_{\Sigma_{\tau}} J_{\mu}^{N}(\Phi) n_{\Sigma_{\tau}}^{\mu}+\int_{\mathscr{H}\left(\tau_{0}, \tau\right)} J_{\mu}^{N}(\Phi) n_{\mathscr{H}^{+}}^{\mu}+\iint_{\mathscr{R}\left(\tau_{0}, \tau\right) \cap\left\{r \leq r_{Y}^{-}\right\}} K^{N}(\Phi)+\iint_{\mathscr{R}\left(\tau^{\prime}, \tau\right)} K^{X_{0}}(\Phi) \leq C \int_{\Sigma_{\tau_{0}}} J_{\mu}^{N}(\Phi) n_{\Sigma_{\tau_{0}}}^{\mu} ;$

(2) The decay of nondegenerate energy:

$$
\begin{gathered}
\tau^{2} \int_{\Sigma_{\tau} \cap\{r \leq \gamma \tau\}} J_{\mu}^{N}(\Phi) n_{\Sigma_{\tau}}^{\mu}+c \int_{\Sigma_{\tau}} J_{\mu}^{Z+N, w^{Z}}(\Phi) n_{\Sigma_{\tau}}^{\mu} \leq C \tau^{1+\eta} \sum_{m=0}^{1} \int_{\Sigma_{\tau_{0}}} J_{\mu}^{Z+C N, w^{Z}}\left(\partial_{t^{*}}^{m} \Phi\right) n_{\Sigma_{\tau_{0}}}^{\mu}, \\
\tau^{2} \int_{\Sigma_{\tau} \cap\{r \leq \gamma \tau\}} J_{\mu}^{N}(\Phi) n_{\Sigma_{\tau}}^{\mu}+c \int_{\Sigma_{\tau}} J_{\mu}^{Z+N, w^{Z}}(\Phi) n_{\Sigma_{\tau}}^{\mu} \leq C \tau^{\eta} \sum_{m=0}^{2} \int_{\Sigma_{\tau_{0}}} J_{\mu}^{Z+C N, w^{Z}}\left(\partial_{t^{*}}^{m} \Phi\right) n_{\Sigma_{\tau_{0}}}^{\mu} .
\end{gathered}
$$

(3) The decay of local integrated energy: For $\tau^{\prime} \leq \tau \leq(1.1) \tau^{\prime}$,

$$
\begin{aligned}
& \iint_{\mathscr{R}\left(\tau^{\prime}, \tau\right) \cap\left\{r \leq \frac{1}{2} t^{*}\right\}} K^{X_{0}}(\Phi)+ \iint_{\mathscr{R}\left(\tau^{\prime}, \tau\right) \cap\left\{r \leq r_{Y}^{-}\right\}} K^{N}(\Phi) \leq C \tau^{-2+\eta} \sum_{m=0}^{2} \int_{\Sigma_{\tau_{0}}} J_{\mu}^{Z+C N, w^{Z}}\left(\partial_{t^{*}}^{m} \Phi\right) n_{\Sigma_{\tau_{0}}}^{\mu}, \\
& \iint_{\mathscr{R}\left(\tau^{\prime}, \tau\right) \cap\left\{r \leq \frac{1}{2} t^{*}\right\}} K^{X_{1}}(\Phi) \leq C \tau^{-2+\eta} \sum_{m=0}^{3} \int_{\Sigma_{\tau_{0}}} J_{\mu}^{Z+C N, w^{Z}}\left(\partial_{t^{*}}^{m} \Phi\right) n_{\Sigma_{\tau_{0}}}^{\mu} .
\end{aligned}
$$


Proof. Part 1 follows directly from Proposition 25. Part 2 contains two statements. The second is a restatement of Proposition 42. The first is evident from the proof of Proposition 42. Part 3 again has two statements. For the first, we revisit the proof of Proposition 42. The bootstrap assumptions are true; hence it holds. For the second statement, we note by comparing Propositions 33 and 34 that $K^{X_{1}}$ can be estimated in the same way as $K^{X_{0}}$ except for an extra derivative. The second statement in 3 can then be proved by rerunning the argument in Proposition 42 with an extra derivative.

\section{Estimates for $\hat{Y} \Phi$ and elliptic estimates}

Away from the event horizon, we can control all higher order derivatives simply by commuting with $\partial_{t^{*}}$ and using standard elliptic estimates. We write down a general version of the estimates in which we have inhomogeneous terms.

Proposition 44. Suppose $\square_{g_{K}} \Phi=$ G. For $m \geq 1$ and for any $\alpha$, we have

(1) the boundedness of weighted energy,

$$
\int_{\Sigma_{\tau} \cap\left\{r \geq r_{Y}^{-}\right\}} r^{\alpha}\left(D^{m} \Phi\right)^{2} \leq C_{\alpha, m}\left(\sum_{j=0}^{m-1} \int_{\Sigma_{\tau}} r^{\alpha} J_{\mu}^{N}\left(\partial_{t^{*}}^{j} \Phi\right) n_{\Sigma_{\tau}}^{\mu}+\sum_{j=0}^{m-2} \int_{\Sigma_{\tau}} r^{\alpha}\left(D^{j} G\right)^{2}\right),
$$

(2) and the boundedness of local energy, that is, for any $0<\gamma<\gamma^{\prime}$,

$$
\begin{array}{r}
\int_{\Sigma_{\tau} \cap\left\{r_{Y}^{-} \leq r \leq \gamma t^{*}\right\}} r^{\alpha}\left(D^{m} \Phi\right)^{2} \leq C_{\alpha, m, \gamma, \gamma^{\prime}}\left(\sum_{j=0}^{m-1} \int_{\Sigma_{\tau} \cap\left\{r \leq \gamma^{\prime} t^{*}\right\}} r^{\alpha} J_{\mu}^{N}\left(\partial_{t^{*}}^{j} \Phi\right) n_{\Sigma_{\tau}}^{\mu}+\tau^{\alpha-\beta-2} \int_{\Sigma_{\tau}} r^{\beta} J_{\mu}^{N}(\Phi) n_{\Sigma_{\tau}}^{\mu}\right. \\
\left.+\sum_{j=0}^{m-2} \int_{\Sigma_{\tau} \cap\left\{r \leq \gamma^{\prime} t^{*}\right\}} r^{\alpha}\left(D^{j} G\right)^{2}\right) .
\end{array}
$$

Proof. This is obvious for $m=1$ (even without the restriction $r \geq r_{Y}^{-}$). We will proceed by induction. Take $\delta \ll\left(r_{Y}^{-}-r_{+}\right) / 4$. Assume

$$
\sum_{j=1}^{m-1} \int_{\Sigma_{\tau} \cap\left\{r \geq r_{Y}^{-}-2 \delta\right\}} r^{\alpha}\left(D^{j} \Phi\right)^{2} \leq C\left(\sum_{j=0}^{m-2} \int_{\Sigma_{\tau}} r^{\alpha} J_{\mu}^{N}\left(\partial_{t^{*}}^{j} \Phi\right) n_{\Sigma_{\tau}}^{\mu}+\sum_{j=0}^{m-3} \int_{\Sigma_{\tau}} r^{\alpha}\left(D^{j} G\right)^{2}\right) .
$$

We want to show

$$
\int_{\Sigma_{\tau} \cap\left\{r \geq r_{Y}^{-}-\delta\right\}} r^{\alpha}\left(D^{m} \Phi\right)^{2} \leq C\left(\sum_{j=0}^{m-1} \int_{\Sigma_{\tau}} r^{\alpha} J_{\mu}^{N}\left(\partial_{t^{*}}^{j} \Phi\right) n_{\Sigma_{\tau}}^{\mu}+\sum_{j=0}^{m-2} \int_{\Sigma_{\tau}} r^{\alpha}\left(D^{j} G\right)^{2}\right),
$$

which would then imply the conclusion. Denote by $\Delta_{g_{K}}$ the Laplace-Beltrami operator for the metric $g_{K}$ restricted on the spacelike hypersurface on which $t^{*}$ is constant. Since $\partial_{t^{*}}$ is Killing, the operator is defined independent of $t^{*}$. Then we have

$$
\left|\left[\Delta_{g_{K}}, D^{k}\right] \Phi\right| \leq C \sum_{j=1}^{k+1}\left|D^{j} \Phi\right|
$$


Denote by $\nabla$ the spatial derivatives with respect to the spatial coordinate variables in the Schwarzschild $\left(t_{S}^{*}, r_{S}, x_{S}^{1}, x_{S}^{2}\right)$ coordinate system. On the set $\left\{r \geq r_{Y}^{-}-\left(r_{Y}^{-}-r_{+}\right) / 4\right\}, \Delta_{g_{K}}$ is elliptic and therefore controls all spatial derivatives:

$$
\begin{aligned}
& \int_{\Sigma_{\tau} \cap\left\{r \geq r_{Y}^{-}-\delta\right\}} r^{\alpha}\left(D^{m} \Phi\right)^{2} \\
& \quad \leq C \int_{\Sigma_{\tau} \cap\left\{r \geq r_{Y}^{-}-2 \delta\right\}} r^{\alpha}\left(\left(\Delta_{g_{K}} D^{m-2} \Phi\right)^{2}+\left(D^{m-1} \Phi\right)^{2}+\left(\partial_{t^{*}}^{m-1} \nabla \Phi\right)^{2}+\left(\partial_{t^{*}}^{m} \Phi\right)^{2}\right) \\
& \quad \leq C \int_{\Sigma_{\tau} \cap\left\{r \geq r_{Y}^{-}-2 \delta\right\}} r^{\alpha}\left(\left(D^{m-2} \Delta_{g_{K}} \Phi\right)^{2}+\sum_{j=1}^{m-1}\left(D^{j} \Phi\right)^{2}+r^{-2} \Phi^{2}+\left(\partial_{t^{*}}^{m-1} \nabla \Phi\right)^{2}+\left(\partial_{t^{*}}^{m} \Phi\right)^{2}\right)
\end{aligned}
$$

The last two terms are obviously bounded by $C \int_{\Sigma_{\tau}} J_{\mu}^{N}\left(\partial_{t^{*}}^{m-1} \Phi\right) n_{\Sigma_{\tau}}^{\mu}$. The second term can be bounded using the induction hypothesis. The third term can be bounded using the Hardy inequality in Proposition 21. Finally, to estimate the first term we use the equation $\square_{g_{K}} \Phi=G$. Then, by the form of the Kerr metric, $\Delta_{g_{K}} \Phi=G-g^{t^{*} t^{*}} \partial_{t^{*}}^{2} \Phi-2 g^{t^{*} \phi^{*}} \partial_{t^{*}} \partial_{\phi^{*}} \Phi$. Therefore,

$$
\begin{aligned}
\left.\int_{\Sigma_{\tau} \cap\{r} \geq r_{Y}^{-}-2 \delta\right\} & r^{\alpha}\left(D^{m-2} \Delta_{g_{K}} \Phi\right)^{2} \\
& \leq C \int_{\Sigma_{\tau} \cap\left\{r \geq r_{Y}^{-}-2 \delta\right\}} r^{\alpha}\left(\left(D^{m-1} \partial_{t^{*}} \Phi\right)^{2}+\left(D^{m-2} G\right)^{2}\right) \\
& \leq C\left(\sum_{j=0}^{m-1} \int_{\Sigma_{\tau}} r^{\alpha} J_{\mu}^{N}\left(\partial_{t^{*}}^{j} \Phi\right) n_{\Sigma_{\tau}}^{\mu}+\sum_{j=0}^{m-2} \int_{\Sigma_{\tau}} r^{\alpha}\left(D^{j} G\right)^{2}\right),
\end{aligned}
$$

where at the last step we have used the induction hypothesis for $\partial_{t^{*}} \Phi$. We have thus proved the boundedness of weighted energy. To prove the second part of the proposition, consider the function $\chi(r / \tau) \Phi(\tau)$ for a fixed time $t^{*}=\tau$, where $\chi: \mathbb{R}_{\geq 0} \rightarrow \mathbb{R}_{\geq 0}$ is supported in $\left\{x \leq \gamma^{\prime}\right\}$ and is identically 1 in $\{x \leq \gamma\}$. Now

$$
\square_{g_{K}} \Phi=\chi G+\tau^{-1} \tilde{\chi} \partial_{r} \Phi+\tau^{-2} \tilde{\chi} \Phi
$$

where $\tilde{\chi}$ and $\tilde{\tilde{\chi}}$ are supported in $\left\{\gamma \leq t^{*} / r \leq \gamma^{\prime}\right\}$. Thus, by the estimate just proved,

$$
\begin{aligned}
& \int_{\Sigma_{\tau} \cap\left\{r_{Y}^{-} \leq r \leq \gamma t^{*}\right\}} r^{\alpha}\left(D^{m} \Phi\right)^{2} \\
& \quad \leq C_{\alpha}\left(\sum_{j=0}^{m-1} \int_{\Sigma_{\tau} \cap\left\{r \leq \gamma^{\prime} t^{*}\right\}} r^{\alpha} J_{\mu}^{N}\left(\partial_{t^{*}}^{j} \Phi\right) n_{\Sigma_{\tau}}^{\mu}+\int_{\Sigma_{\tau} \cap\left\{\gamma t \leq r \leq \gamma^{\prime} t^{*}\right\}} r^{\alpha} \tau^{-4} \Phi^{2}+\sum_{j=0}^{m-2} \int_{\Sigma_{\tau} \cap\left\{r \leq \gamma^{\prime} t^{*}\right\}} r^{\alpha}\left(D^{j} G\right)^{2}\right) \\
& \quad \leq C_{\alpha}\left(\sum_{j=0}^{m-1} \int_{\Sigma_{\tau} \cap\left\{r \leq \gamma^{\prime} t^{*}\right\}} r^{\alpha} J_{\mu}^{N}\left(\partial_{t^{*}}^{j} \Phi\right) n_{\Sigma_{\tau}}^{\mu}+\tau^{\alpha-\beta-2} \int_{\Sigma_{\tau}} r^{\beta} J_{\mu}^{N}(\Phi) n_{\Sigma_{\tau}}^{\mu}+\sum_{j=0}^{m-2} \int_{\Sigma_{\tau} \cap\left\{r \leq \gamma^{\prime} t^{*}\right\}} r^{\alpha}\left(D^{j} G\right)^{2}\right),
\end{aligned}
$$

by the Hardy inequality in Proposition 21. 
Remark. The boundedness of local energy should be seen as a decay result because for example for the homogeneous equation, the right-hand side of the inequality decays.

Near the event horizon, higher order derivatives can be controlled by commuting with the red-shift vector field as in [Dafermos and Rodnianski 2008; 2011]. The computation here will be completely local, that is, only in the region $\left\{r \leq r_{Y}^{-}\right\}$.

We have the following estimate for higher order derivatives:

Proposition 45. Suppose $\square_{g_{K}} \Phi=$ G. For every $m \geq 1$,

$$
\int_{\Sigma_{\tau} \cap\left\{r \leq r_{Y}^{-}\right\}}\left(D^{m} \Phi\right)^{2} \leq C\left(\sum_{j+k \leq m-1} \int_{\Sigma_{\tau} \cap\left\{r \leq r_{Y}^{-}\right\}} J_{\mu}^{N}\left(\partial_{t^{*}}^{j} \hat{Y}^{k} \Phi\right) n_{\Sigma_{\tau}}^{\mu}+\sum_{j=0}^{m-2} \int_{\Sigma_{\tau} \cap\left\{r \leq r_{Y}^{-}\right\}}\left(D^{j} G\right)^{2}\right) .
$$

Proof. This is obvious for $m=1$. We will proceed by induction. Suppose, for some $m \geq 2$ that

$$
\sum_{j=0}^{m-1} \int_{\Sigma_{\tau} \cap\left\{r \leq r_{Y}^{-}\right\}}\left(D^{j} \Phi\right)^{2} \leq C\left(\sum_{j+k \leq m-2} \int_{\Sigma_{\tau} \cap\left\{r \leq r_{Y}^{-}\right\}} J_{\mu}^{N}\left(\partial_{t^{*}}^{j} \hat{Y}^{k} \Phi\right) n_{\Sigma_{\tau}}^{\mu}+\sum_{j=0}^{m-3} \int_{\Sigma_{\tau} \cap\left\{r \leq r_{Y}^{-}\right\}}\left(D^{j} G\right)^{2}\right) .
$$

Since $\square_{g_{K}}\left(\partial_{t^{*}} \Phi\right)=\partial_{t^{*}} G$, this immediately implies

$$
\int_{\Sigma_{\tau} \cap\left\{r \leq r_{Y}^{-}\right\}}\left(\partial_{t^{*}} D^{m-1} \Phi\right)^{2} \leq C\left(\sum_{j+k \leq m-1} \int_{\Sigma_{\tau} \cap\left\{r \leq r_{Y}^{-}\right\}} J_{\mu}^{N}\left(\partial_{t^{*}}^{j} \hat{Y}^{k} \Phi\right) n_{\Sigma_{\tau}}^{\mu}+\sum_{j=0}^{m-2} \int_{\Sigma_{\tau} \cap\left\{r \leq r_{Y}^{-}\right\}}\left(D^{j} G\right)^{2}\right) .
$$

Since $\square_{g_{K}} \Phi=G$, we have $\square_{g_{K}}(\hat{Y} \Phi)=\hat{Y} G+O(1)\left(D^{2} \Phi+D \Phi\right)$. Then using the induction hypothesis (18) (both on $\hat{Y} \Phi$ and $\Phi$ ), we have

$$
\begin{aligned}
& \sum_{j=0}^{m-1} \int_{\Sigma_{\tau} \cap\left\{r \leq r_{Y}^{-}\right\}}\left(D^{j} \hat{Y} \Phi\right)^{2} \\
& \quad \leq C\left(\sum_{j+k \leq m-2} \int_{\Sigma_{\tau} \cap\left\{r \leq r_{Y}^{-}\right\}} J_{\mu}^{N}\left(\partial_{t^{*}}^{j} \hat{Y}^{k+1} \Phi\right) n_{\Sigma_{\tau}}^{\mu}+\sum_{j=0}^{m-3} \int_{\Sigma_{\tau}}\left(D^{j} \hat{Y} G\right)^{2}+\sum_{j=0}^{m-1} \int_{\Sigma_{\tau} \cap\left\{r \leq r_{Y}^{-}\right\}}\left(D^{j} \Phi\right)^{2}\right) \\
& \quad \leq C\left(\sum_{j+k \leq m-1} \int_{\Sigma_{\tau} \cap\left\{r \leq r_{Y}^{-}\right\}} J_{\mu}^{N}\left(\partial_{t^{*}}^{j} \hat{Y}^{k} \Phi\right) n_{\Sigma_{\tau}}^{\mu}+\sum_{j=0}^{m-2} \int_{\Sigma_{\tau} \cap\left\{r \leq r_{Y}^{-}\right\}}\left(D^{j} G\right)^{2}\right) .
\end{aligned}
$$

Using the null frame $\left\{\hat{V}, \hat{Y}, E_{1}, E_{2}\right\}$,

$$
\square_{g_{K}}\left(D^{m-2} \Phi\right)=-4 \nabla_{\hat{Y}} \nabla_{\hat{V}} D^{m-2} \Phi+\not \not D^{m-2} \Phi+P_{1} D^{m-2} \Phi,
$$

where $P_{1}$ denotes a first order differential operator. Notice that we also have

$$
\left|\square_{g_{K}}\left(D^{m-2} \Phi\right)\right|=\left|\left[\square_{g_{K}}, D^{m-2}\right] \Phi+D^{m-2} G\right| \leq C\left(\sum_{j=0}^{m-1}\left|D^{j} \Phi\right|+\left|D^{m-2} G\right|\right) .
$$


Now using a standard $L^{2}$ elliptic estimate on the sphere, we have

$$
\int_{\mathbb{S}^{2}}\left|\not^{2} D^{m-2} \Phi\right|^{2} d A \leq C \int_{\mathbb{S}^{2}}\left(\left(D^{m-2} G\right)^{2}+\sum_{j=0}^{m-1}\left(D^{j} \Phi\right)^{2}+\left(D^{m-1} \nabla_{\hat{Y}} \Phi\right)^{2}\right) d A
$$

where we notice that the constant can be chosen uniformly because the metric on the sphere is everywhere close to that of the standard metric. Therefore, after integrate over $\left\{r_{+} \leq r \leq r_{Y}^{-}\right\}$and applying (18) and (20), we have

$$
\begin{aligned}
\int_{\Sigma_{\tau} \cap\left\{r \leq r_{Y}^{-}\right\}}\left|\not^{2} D^{m-2} \Phi\right|^{2} & \leq C \int_{\Sigma_{\tau} \cap\left\{r \leq r_{Y}^{-}\right\}}\left(\left(D^{m-2} G\right)^{2}+\sum_{j=0}^{m-1}\left(D^{j} \Phi\right)^{2}+\left(D^{m-1} \nabla_{\hat{Y}} \Phi\right)^{2}\right) \\
& \leq C\left(\sum_{j+k \leq m-1} \int_{\Sigma_{\tau} \cap\left\{r \leq r_{Y}^{-}\right\}} J_{\mu}^{N}\left(\partial_{t^{*}}^{j} \hat{Y}^{k} \Phi\right) n_{\Sigma_{\tau}}^{\mu}+\sum_{j=0}^{m-2} \int_{\Sigma_{\tau} \cap\left\{r \leq r_{Y}^{-}\right\}}\left(D^{j} G\right)^{2}\right) .
\end{aligned}
$$

Combining (19), (20) and (21), we have

$$
\int_{\Sigma_{\tau} \cap\left\{r \leq r_{Y}^{-}\right\}}\left(D^{m} \Phi\right)^{2} \leq C\left(\sum_{j+k \leq m} \int_{\Sigma_{\tau} \cap\left\{r \leq r_{Y}^{-}\right\}} J_{\mu}^{N}\left(\partial_{t^{*}}^{j} \hat{Y}^{k} \Phi\right) n_{\Sigma_{\tau}}^{\mu}+\sum_{j=0}^{m-2} \int_{\Sigma_{\tau} \cap\left\{r \leq r_{Y}^{-}\right\}}\left(D^{j} G\right)^{2}\right) .
$$

We show that the currents associated to $\hat{Y}^{k} \Phi$ can actually be controlled. Again, in view of the nonlinear problem, we work in the setting of an inhomogeneous equation.

Proposition 46. Suppose $\square_{g_{K}} \Phi=$ G. For every $k \geq 0$,

$$
\begin{aligned}
\int_{\Sigma_{\tau} \cap\left\{r \leq r_{Y}^{+}\right\}} J_{\mu}^{N}\left(\hat{Y}^{k} \Phi\right) n_{\Sigma_{\tau}}^{\mu}+\int_{\mathscr{H}\left(\tau^{\prime}, \tau\right)} J_{\mu}^{N}\left(\hat{Y}^{k} \Phi\right) n_{\Sigma_{\tau}}^{\mu}+\iint_{\mathscr{R}\left(\tau^{\prime}, \tau\right) \cap\left\{r \leq r_{Y}^{-}\right\}} K^{N}\left(\hat{Y}^{k} \Phi\right) \\
\leq C\left(\sum_{j+m \leq k} \int_{\Sigma_{\tau^{\prime}} \cap\left\{r \leq r_{Y}^{+}\right\}} J_{\mu}^{N}\left(\partial_{t^{*}}^{j} \hat{Y}^{m} \Phi\right) n_{\Sigma_{\tau^{\prime}}}^{\mu}+\sum_{j=0}^{k} \int_{\Sigma_{\tau} \cap\left\{r \leq r_{Y}^{+}\right\}} J_{\mu}^{N}\left(\partial_{t^{*}}^{j} \Phi\right) n_{\Sigma_{\tau}}^{\mu}\right. \\
\left.\quad+\sum_{j=0}^{k} \iint_{\mathscr{R}\left(\tau^{\prime}, \tau\right) \cap\left\{r \leq \frac{23}{8} M\right\}}\left(\Phi^{2}+J_{\mu}^{N}\left(\partial_{t^{*}}^{j} \Phi\right) n_{\Sigma_{t^{*}}}^{\mu}\right)+\sum_{j=0}^{k} \iint_{\mathscr{R}\left(\tau^{\prime}, \tau\right) \cap\left\{r \leq \frac{23}{8} M\right\}}\left(D^{j} G\right)^{2}\right) .
\end{aligned}
$$

Proof. We prove the proposition by induction on $k$. The $k=0$ case is trivial because the right-hand side simply contains more terms than the left hand side. We suppose the proposition is true for $k \leq k_{0}-1$ for some $k_{0} \geq 1$. Commuting $\square_{g_{K}}$ with $\hat{Y}$ for $k_{0}$ times, we get

$$
\square_{g_{K}} \hat{Y}^{k_{0}} \Phi=\kappa^{k_{0}} \hat{Y}^{k_{0}+1} \Phi+O(1) \hat{Y}^{k_{0}} \partial_{t^{*}} \Phi+O(\epsilon) D^{k_{0}+1} \Phi+O(1) \sum_{j=1}^{k_{0}} D^{j} \Phi+\hat{Y}^{k_{0}} G
$$


We now use the energy identity for the vector field $N$, that is, Proposition 9 for $\hat{Y}^{k} \Phi$. Notice that $\hat{Y}$ is supported in $\left\{r \leq r_{Y}^{+}\right\}$and therefore each term is supported in the same set. Then

$$
\begin{aligned}
& \int_{\Sigma_{\tau} \cap\left\{r \leq r_{Y}^{+}\right\}} J_{\mu}^{N}\left(\hat{Y}^{k_{0}} \Phi\right) n_{\Sigma_{\tau}}^{\mu}+\int_{\mathscr{H}\left(\tau_{0}, \tau\right)} J_{\mu}^{N}\left(\hat{Y}^{k_{0}} \Phi\right) n_{\Sigma_{\tau}}^{\mu}+\iint_{\mathscr{R}\left(\tau_{0}, \tau\right) \cap\left\{r \leq r_{Y}^{-}\right\}} K^{N}\left(\hat{Y}^{k_{0}} \Phi\right) \\
& =\int_{\Sigma_{\tau_{0}}} J_{\mu}^{N}\left(\hat{Y}^{k_{0}} \Phi\right) n_{\Sigma_{\tau_{0}}}^{\mu}+e \iint_{\mathscr{R}\left(\tau_{0}, \tau\right) \cap\left\{r_{Y}^{-} \leq r \leq r_{Y}^{+}\right\}} K^{Y}\left(\hat{Y}^{k_{0}} \Phi\right) \\
& \quad+\iint_{\mathscr{R}\left(\tau_{0}, \tau\right) \cap\left\{r \leq r_{Y}^{+}\right\}}\left(\partial_{t^{*}} \hat{Y}^{k_{0}} \Phi+e \hat{Y}^{k_{0}+1} \Phi\right)\left(-\kappa^{k_{0}} \hat{Y}^{k_{0}+1} \Phi+O(1) \hat{Y}^{k_{0}} \partial_{t^{*}} \Phi\right. \\
& \left.\quad+O(\epsilon) D^{k_{0}+1} \Phi+O(1) \sum_{j=1}^{k_{0}} D^{j} \Phi+\hat{Y}^{k} G\right) .
\end{aligned}
$$

The crucial observation in [Dafermos and Rodnianski 2011] is that one of the inhomogeneous terms has a good sign and thus gives

$$
\begin{aligned}
& \int_{\Sigma_{\tau}} J_{\mu}^{N}\left(\hat{Y}^{k_{0}} \Phi\right) n_{\Sigma_{\tau}}^{\mu}+\int_{\mathscr{H}\left(\tau^{\prime}, \tau\right)} J_{\mu}^{N}\left(\hat{Y}^{k_{0}} \Phi\right) n_{\Sigma_{\tau}}^{\mu}+\iint_{\mathscr{R}\left(\tau^{\prime}, \tau\right) \cap\left\{r \leq r_{Y}^{-}\right\}} K^{N}\left(\hat{Y}^{k_{0}} \Phi\right)+\iint_{\mathscr{R}\left(\tau^{\prime}, \tau\right)}\left(\hat{Y}^{k_{0}+1} \Phi\right)^{2} \\
& \leq C\left(\int_{\Sigma_{\tau^{\prime}} \cap\left\{r \leq r_{Y}^{+}\right\}} J_{\mu}^{N}\left(\hat{Y}^{k_{0}} \Phi\right) n_{\Sigma_{\tau_{0}}}^{\mu}+\iint_{\mathscr{R}_{\left(\tau^{\prime}, \tau\right) \cap\left\{r_{Y}^{-} \leq r \leq r_{Y}^{+}\right\}}} K^{N}\left(\hat{Y}^{k_{0}} \Phi\right)+\epsilon \iint_{\Re_{R}\left(\tau^{\prime}, \tau\right) \cap\left\{r \leq r_{Y}^{+}\right\}}\left(D^{k_{0}+1} \Phi\right)^{2}\right. \\
& \left.+\iint_{\mathscr{R}\left(\tau^{\prime}, \tau\right) \cap\left\{r \leq r_{Y}^{+}\right\}} J_{\mu}^{N}\left(\partial_{t^{*}} \hat{Y}^{k_{0}-1} \Phi\right) n_{\Sigma_{t^{*}}}^{\mu}+\sum_{j=1}^{k_{0}} \iint_{\mathscr{R}^{\prime}\left(\tau^{\prime}, \tau\right) \cap\left\{r \leq r_{Y}^{+}\right\}}\left(D^{j} \Phi\right)^{2}+\iint_{\mathscr{R}^{\prime}\left(\tau^{\prime}, \tau\right) \cap\left\{r \leq r_{Y}^{+}\right\}}\left(\hat{Y}^{k_{0}} G\right)^{2}\right) \\
& \leq C\left(\int_{\Sigma_{\tau^{\prime}} \cap\left\{r \leq r_{Y}^{+}\right\}} J_{\mu}^{N}\left(\hat{Y}^{k_{0}} \Phi\right) n_{\Sigma_{\tau_{0}}}^{\mu}+\sum_{j=1}^{k_{0}+1} \iint_{\mathscr{R}^{\prime}\left(\tau^{\prime}, \tau\right) \cap\left\{r_{Y}^{-} \leq r \leq r_{Y}^{+}\right\}}\left(D^{j} \Phi\right)^{2}+\epsilon \iint_{\mathscr{R}_{\left(\tau^{\prime}, \tau\right) \cap\left\{r \leq r_{Y}^{-}\right\}}}\left(D^{k_{0}+1} \Phi\right)^{2}\right. \\
& \left.+\iint_{\mathscr{R}\left(\tau^{\prime}, \tau\right) \cap\left\{r \leq r_{Y}^{-}\right\}} J_{\mu}^{N}\left(\partial_{t^{*}} \hat{Y}^{k_{0}-1} \Phi\right) n_{\Sigma_{t^{*}}}^{\mu}+\sum_{j=1}^{k_{0}} \iint_{\mathscr{R}\left(\tau^{\prime}, \tau\right) \cap\left\{r \leq r_{Y}^{-}\right\}}\left(D^{j} \Phi\right)^{2}+\iint_{\mathscr{R}_{\left(\tau^{\prime}, \tau\right) \cap\left\{r \leq r_{Y}^{+}\right\}}}\left(\hat{Y}^{k_{0}} G\right)^{2}\right) .
\end{aligned}
$$

Using Proposition 44 with an appropriate cutoff, we have

$$
\begin{aligned}
& \sum_{j=1}^{k_{0}+1} \iint_{\mathscr{R}\left(\tau^{\prime}, \tau\right) \cap\left\{r_{Y}^{-} \leq r \leq r_{Y}^{+}\right\}}\left(D^{j} \Phi\right)^{2} \\
& \leq C\left(\sum_{j=0}^{k_{0}} \iint_{\mathscr{R}\left(\tau^{\prime}, \tau\right) \cap\left\{r \leq \frac{23}{8} M\right\}}\left(\Phi^{2}+J_{\mu}^{N}\left(\partial_{t^{*}}^{j} \Phi\right) n_{\Sigma_{t^{*}}}^{\mu}\right)+\sum_{j=0}^{k_{0}-1} \iint_{\mathscr{R}\left(\tau^{\prime}, \tau\right) \cap\left\{r \leq \frac{23}{8} M\right\}}\left(D^{j} G\right)^{2}\right) .
\end{aligned}
$$


Using Proposition 45, we get

$$
\begin{aligned}
& \sum_{j=1}^{k_{0}} \iint_{\mathscr{R}\left(\tau^{\prime}, \tau\right) \cap\left\{r \leq r_{Y}^{-}\right\}}\left(D^{j} \Phi\right)^{2} \\
& \quad \leq C\left(\sum_{j+m \leq k_{0}-1} \iint_{\mathscr{R}\left(\tau^{\prime}, \tau\right) \cap\left\{r \leq r_{Y}^{-}\right\}} J_{\mu}^{N}\left(\partial_{t^{*}}^{j} \hat{Y}^{m} \Phi\right) n_{\Sigma_{t^{*}}}^{\mu}+\sum_{j=0}^{k_{0}-2} \iint_{\mathscr{R}\left(\tau^{\prime}, \tau\right) \cap\left\{r \leq r_{Y}^{-}\right\}}\left(D^{j} G\right)^{2}\right) \\
& \quad \leq C\left(\sum_{j+m \leq k_{0}-1} \int_{\Sigma_{\tau^{\prime}} \cap\left\{r \leq r_{Y}^{+}\right\}} J_{\mu}^{N}\left(\partial_{t^{*}}^{j} \hat{Y}^{m} \Phi\right) n_{\Sigma_{\tau^{\prime}}}^{\mu}+\sum_{j=0}^{k-1} \int_{\Sigma_{\tau} \cap\left\{r \leq r_{Y}^{+}\right\}} J_{\mu}^{N}\left(\partial_{t^{*}}^{j} \Phi\right) n_{\Sigma_{\tau}}^{\mu}\right. \\
& \left.\quad+\sum_{j+m \leq k_{0}-1} \iint_{\mathscr{R}\left(\tau^{\prime}, \tau\right) \cap\left\{r \leq \frac{23}{8} M\right\}} J_{\mu}^{N}\left(\partial_{t^{*}}^{j} \hat{Y}^{m} \Phi\right) n_{\Sigma_{t^{*}}}^{\mu}+\sum_{j=0}^{k_{0}-1} \iint_{\mathscr{R}\left(\tau^{\prime}, \tau\right) \cap\left\{r \leq \frac{23}{8} M\right\}}\left(D^{j} G\right)^{2}\right),
\end{aligned}
$$

using the induction hypothesis (on $\partial_{t^{*}}^{m} \Phi$ instead of $\Phi$ ) at the last step. Similarly, using Proposition 45,

$$
\begin{aligned}
& \epsilon \iint_{\mathscr{R}\left(\tau^{\prime}, \tau\right) \cap\left\{r \leq r_{Y}^{-}\right\}}\left(D^{k_{0}+1} \Phi\right)^{2} \\
& \leq C \epsilon\left(\sum_{j+m \leq k_{0}} \iint_{\mathscr{R}\left(\tau^{\prime}, \tau\right) \cap\left\{r \leq r_{Y}^{-}\right\}} J_{\mu}^{N}\left(\partial_{t^{*}}^{j} \hat{Y}^{m} \Phi\right) n_{\Sigma_{t^{*}}}^{\mu}+\sum_{j=0}^{k-1} \iint_{\mathscr{R}\left(\tau^{\prime}, \tau\right) \cap\left\{r \leq r_{Y}^{-}\right\}}\left(D^{j} G\right)^{2}\right) \\
& \leq C \epsilon\left(\iint_{\mathscr{R}\left(\tau^{\prime}, \tau\right) \cap\left\{r \leq r_{Y}^{+}\right\}} J_{\mu}^{N}\left(\hat{Y}^{k_{0}} \Phi\right) n_{\Sigma_{t^{*}}}^{\mu}+\sum_{j=0}^{k_{0}} \int_{\Sigma_{\tau^{\prime}} \cap\left\{r \leq r_{Y}^{+}\right\}} J_{\mu}^{N}\left(\partial_{t^{*}}^{j} \Phi\right) n_{\Sigma_{\tau^{\prime}}}^{\mu}\right. \\
& \left.+\sum_{j=0}^{k_{0}} \iint_{\mathscr{R}\left(\tau^{\prime}, \tau\right) \cap\left\{r \leq \frac{23}{8} M\right\}} J_{\mu}^{N}\left(\partial_{t^{*}}^{j} \Phi\right) n_{\Sigma_{t^{*}}}^{\mu}+\sum_{j=0}^{k_{0}} \iint_{\mathscr{R}\left(\tau^{\prime}, \tau\right) \cap\left\{r \leq \frac{23}{8} M\right\}}\left(D^{j} G\right)^{2}\right),
\end{aligned}
$$

where again the induction hypotheses is used at the last step. All these together give

$$
\begin{aligned}
\int_{\Sigma_{\tau}} J_{\mu}^{N}\left(\hat{Y}^{k_{0}} \Phi\right) n_{\Sigma_{\tau}}^{\mu}+\int_{\mathscr{H}\left(\tau_{0}, \tau\right)} J_{\mu}^{N}\left(\hat{Y}^{k_{0}} \Phi\right) n_{\Sigma_{\tau}}^{\mu}+\iint_{\mathscr{R}\left(\tau_{0}, \tau\right) \cap\left\{r \leq r_{Y}^{-}\right\}} K^{N}\left(\hat{Y}^{k_{0}} \Phi\right) \\
\leq C\left(\sum_{j+m \leq k_{0}} \int_{\Sigma_{\tau^{\prime}} \cap\left\{r \leq r_{Y}^{+}\right\}} J_{\mu}^{N}\left(\partial_{t^{*}}^{j} \hat{Y}^{m} \Phi\right) n_{\Sigma_{\tau^{\prime}}}^{\mu}+\sum_{j=0}^{k_{0}} \int_{\Sigma_{\tau^{\prime}} \cap\left\{r \leq r_{Y}^{+}\right\}} J_{\mu}^{N}\left(\partial_{t^{*}}^{j} \Phi\right) n_{\Sigma_{\tau^{\prime}}}^{\mu}\right. \\
+\sum_{j=0}^{k_{0}} \iint_{\mathscr{R}\left(\tau^{\prime}, \tau\right) \cap\left\{r \leq \frac{23}{8} M\right\}}\left(\Phi^{2}+J_{\mu}^{N}\left(\partial_{t^{*}}^{j} \Phi\right) n_{\Sigma_{t^{*}}}^{\mu}\right)+\sum_{j=0}^{k_{0}} \int_{\mathscr{R}\left(\tau^{\prime}, \tau\right) \cap\left\{r \leq \frac{23}{8} M\right\}}\left(D^{j} G\right)^{2} \\
\left.+\epsilon \iint_{\mathscr{R}\left(\tau^{\prime}, \tau\right) \cap\left\{r \leq r_{Y}^{-}\right\}} J_{\mu}^{N}\left(\hat{Y}^{k_{0}} \Phi\right) n_{\Sigma_{t^{*}}}^{\mu}\right) .
\end{aligned}
$$

The proposition can be proved by noticing that

$$
\iint_{\mathscr{R}\left(\tau^{\prime}, \tau\right) \cap\left\{r \leq r_{Y}^{-}\right\}} J_{\mu}^{N}\left(\hat{Y}^{k_{0}} \Phi\right) n_{\Sigma_{t^{*}}}^{\mu} \leq C \iint_{\mathscr{R}\left(\tau_{0}, \tau\right) \cap\left\{r \leq r_{Y}^{-}\right\}} K^{N}\left(\hat{Y}^{k_{0}} \Phi\right) .
$$


and absorbing the small term to the left-hand side.

We now specialize to the case $\square_{g_{K}} \Phi=0$. The proposition above implies that the behavior of $\hat{Y}^{k} \Phi$ is determined by the behavior of $\partial_{t^{*}}^{m} \Phi$ in the region $\left\{r \leq \frac{23}{8} M\right\}$.

Proposition 47. Fix $k \geq 0$. Suppose $\square_{g_{K}} \Phi=0$ and suppose for some constants $\alpha, B>0$ (independents of $\tau$ ) that

$$
\sum_{j=0}^{k} \int_{\Sigma_{\tau} \cap\left\{r \leq \frac{23}{8} M\right\}}\left(\Phi^{2}+J_{\mu}^{N}\left(\partial_{t^{*}}^{j} \Phi\right) n_{\Sigma_{\tau}}^{\mu}\right) \leq C B \tau^{-\alpha}
$$

Then

$$
\sum_{j+m \leq k} \int_{\Sigma_{\tau}} J_{\mu}^{N}\left(\partial_{t^{*}}^{j} \hat{Y}^{m} \Phi\right) n_{\Sigma_{\tau}}^{\mu} \leq C \tau^{-\alpha}\left(\sum_{j+m \leq k} \int_{\Sigma_{\tau_{0}}} J_{\mu}^{N}\left(\partial_{t^{*}}^{j} \hat{Y}^{m} \Phi\right) n_{\Sigma_{\tau_{0}}}^{\mu}+B\right)
$$

and

$$
\sum_{j+m \leq k} \iint_{\mathscr{R}\left(\tau^{\prime}, \tau\right) \cap\left\{r \leq r_{Y}^{-}\right\}} K^{N}\left(\partial_{t^{*}}^{j} \hat{Y}^{m} \Phi\right) \leq C\left(\tau^{\prime}\right)^{-\alpha}\left(\sum_{j+m \leq k} \int_{\Sigma_{\tau_{0}}} J_{\mu}^{N}\left(\partial_{t^{*}}^{j} \hat{Y}^{m} \Phi\right) n_{\Sigma_{\tau_{0}}}^{\mu}+B\right) .
$$

Remark. In the applications, we will apply this proposition with $B$ being some energy quantity of the initial condition.

Proof. We will proof this with a bootstrap argument. Suppose for all $\tau$ that

$$
\sum_{j+m \leq k} \int_{\Sigma_{\tau} \cap\left\{r \leq r_{Y}^{+}\right\}} J_{\mu}^{N}\left(\partial_{t^{*}}^{j} \hat{Y}^{m} \Phi\right) n_{\Sigma_{\tau}}^{\mu} \leq A \tau^{-\alpha}\left(\sum_{j+m \leq k} \int_{\Sigma_{\tau_{0}}} J_{\mu}^{N}\left(\partial_{t^{*}}^{j} \hat{Y}^{m} \Phi\right) n_{\Sigma_{\tau_{0}}}^{\mu}+B\right) .
$$

This obviously holds initially for any $A \geq 1$ (and in particular independent of $\Phi$ ). By taking $\tau^{\prime}=\tau-K$, for some (large and to be chosen) constant $K$ and $\tau \geq 2 K$, Proposition 46 implies

$$
\begin{aligned}
& \sum_{j+m \leq k}\left(\int_{\Sigma_{\tau} \cap\left\{r \leq r_{Y}^{+}\right\}} J_{\mu}^{N}\left(\partial_{t^{*}}^{j} \hat{Y}^{m} \Phi\right) n_{\Sigma_{\tau}}^{\mu}+\iint_{\mathscr{R}(\tau-K, \tau) \cap\left\{r \leq r_{Y}^{-}\right\}} J_{\mu}^{N}\left(\partial_{t^{*}}^{j} \hat{Y}^{m} \Phi\right) n_{\Sigma_{t^{*}}}^{\mu}\right) \\
& \quad \leq C\left(\sum_{j+m \leq k} \int_{\Sigma_{\tau-K} \cap\left\{r \leq r_{Y}^{+}\right\}} J_{\mu}^{N}\left(\partial_{t^{*}}^{j} \hat{Y}^{m} \Phi\right) n_{\Sigma_{\tau-K}}^{\mu}+\sum_{j=0}^{k} \int_{\Sigma_{\tau} \cap\left\{r \leq r_{Y}^{+}\right\}} J_{\mu}^{N}\left(\partial_{t^{*}}^{j} \Phi\right) n_{\Sigma_{\tau}}^{\mu}\right. \\
& \left.\quad+\sum_{j=0}^{k} \iint_{\mathscr{R}(\tau-K, \tau) \cap\left\{r \leq \frac{23}{8} M\right\}}\left(\Phi^{2}+J_{\mu}^{N}\left(\partial_{t^{*}}^{j} \Phi\right) n_{\Sigma_{t^{*}}}^{\mu}\right)\right) \\
& \quad \leq C\left(\sum_{j+m \leq k} \int_{\Sigma_{\tau-K} \cap\left\{r \leq r_{Y}^{+}\right\}} J_{\mu}^{N}\left(\partial_{t^{*}}^{j} \hat{Y}^{m} \Phi\right) n_{\Sigma_{\tau-K}}^{\mu}+K B \tau^{-\alpha}\right),
\end{aligned}
$$


using the assumption of the proposition and using Proposition 44). Using the bootstrap assumption, we further see that this expression satisfies the bound

$$
\begin{aligned}
& \leq C\left(A(\tau-K)^{-\alpha}\left(\sum_{j+m \leq k} \int_{\Sigma_{\tau_{0}}} J_{\mu}^{N}\left(\partial_{t^{*}}^{j} \hat{Y}^{m} \Phi\right) n_{\Sigma_{\tau_{0}}}^{\mu}+B\right)+K B \tau^{-\alpha}\right) \\
& \leq C \tau^{-\alpha}\left(\sum_{j+m \leq k} A \int_{\Sigma_{\tau_{0}}} J_{\mu}^{N}\left(\partial_{t^{*}}^{j} \hat{Y}^{m} \Phi\right) n_{\Sigma_{\tau_{0}}}^{\mu}+A B+K B\right) .
\end{aligned}
$$

Notice that $C$ is independent of $K$. By selecting a $t^{*}$ slice, we have that for some $\tilde{\tau}$,

$$
\int_{\Sigma_{\tilde{\tau} \cap\left\{r \leq r_{Y}^{-}\right\}}} J_{\mu}^{N}\left(\hat{Y}^{k} \Phi\right) n_{\Sigma_{\tilde{\tau}}}^{\mu} \leq C K^{-1} \tau^{-\alpha}\left(\sum_{j+m \leq k} A \int_{\Sigma_{\tau_{0}}} J_{\mu}^{N}\left(\partial_{t^{*}}^{j} \hat{Y}^{m} \Phi\right) n_{\Sigma_{\tau_{0}}}^{\mu}+A B+K B\right) .
$$

Now apply Proposition 46 on $[\tilde{\tau}, \tau]$ to get

$$
\begin{aligned}
& \sum_{j+m \leq k} \int_{\Sigma_{\tau} \cap\left\{r \leq r_{Y}^{+}\right\}} J_{\mu}^{N}\left(\partial_{t^{*}}^{j} \hat{Y}^{m} \Phi\right) n_{\Sigma_{\tau}}^{\mu} \\
& \leq C\left(\sum_{j+m \leq k} \int_{\Sigma_{\tilde{\tau}} \cap\left\{r \leq r_{Y}^{+}\right\}} J_{\mu}^{N}\left(\partial_{t^{*}}^{j} \hat{Y}^{m} \Phi\right) n_{\Sigma_{\tilde{\tau}}}^{\mu}+\sum_{j=0}^{k} \int_{\Sigma_{\tau} \cap\left\{r \leq r_{Y}^{+}\right\}} J_{\mu}^{N}\left(\partial_{t^{*}}^{j} \Phi\right) n_{\Sigma_{\tau}}^{\mu}\right. \\
& \left.\quad+\sum_{j=0}^{k} \iint_{\mathscr{R}(\tilde{\tau}, \tau) \cap\left\{r \leq \frac{23}{8} M\right\}}\left(\Phi^{2}+J_{\mu}^{N}\left(\partial_{t^{*}}^{j} \Phi\right) n_{\Sigma_{t^{*}}}^{\mu}\right)\right) \\
& \leq C K^{-1} \tau^{-\alpha}\left(\sum_{j+m \leq k} A \int_{\Sigma_{\tau_{0}}} J_{\mu}^{N}\left(\partial_{t^{*}}^{j} \hat{Y}^{m} \Phi\right) n_{\Sigma_{\tau_{0}}}^{\mu}+A B+B K\right)+C B(K+1) \tau^{-\alpha} \\
& \quad \leq C A K^{-1} \tau^{-\alpha} \sum_{j+m \leq k} \int_{\Sigma_{\tau_{0}}} J_{\mu}^{N}\left(\partial_{t^{*}}^{j} \hat{Y}^{m} \Phi\right) n_{\Sigma_{\tau_{0}}}^{\mu}+\left(C A K^{-1}+C K+C\right) B \tau^{-\alpha} .
\end{aligned}
$$

This will improve (22) if we choose $K=4 C$ and $A$ sufficiently large. Hence we have proved

$$
\sum_{j+m \leq k} \int_{\Sigma_{\tau} \cap\left\{r \leq r_{Y}^{+}\right\}} J_{\mu}^{N}\left(\partial_{t^{*}}^{j} \hat{Y}^{m} \Phi\right) n_{\Sigma_{\tau}}^{\mu} \leq C \tau^{-\alpha}\left(\sum_{j+m \leq k} \int_{\Sigma_{\tau_{0}}} J_{\mu}^{N}\left(\partial_{t^{*}}^{j} \hat{Y}^{m} \Phi\right) n_{\Sigma_{\tau_{0}}}^{\mu}+B\right) .
$$

To prove the second statement, we simply use the first statement and Proposition 46.

We can use Corollary 43 to show the decay of $\hat{Y}^{k} \Phi$.

Corollary 48. Suppose $\square_{g_{K}} \Phi=0$. Then for $\tau^{\prime} \leq \tau \leq(1.1) \tau^{\prime}$,

$$
\begin{aligned}
\sum_{j+m \leq k} \int_{\Sigma_{\tau} \cap\left\{r \leq r_{Y}^{+}\right\}} J_{\mu}^{N}\left(\partial_{t^{*}}^{j} \hat{Y}^{m} \Phi\right) & n_{\Sigma_{\tau}}^{\mu} \\
& \leq C \tau^{-2+\eta}\left(\sum_{j+m \leq k} \int_{\Sigma_{\tau_{0}}} J_{\mu}^{N}\left(\partial_{t^{*}}^{j} \hat{Y}^{m} \Phi\right) n_{\Sigma_{\tau_{0}}}^{\mu}+\sum_{j=0}^{k+2} \int_{\Sigma_{\tau_{0}}} J_{\mu}^{Z+C N}\left(\partial_{t^{*}}^{j} \Phi\right) n_{\Sigma_{\tau_{0}}}^{\mu}\right),
\end{aligned}
$$


and

$$
\begin{aligned}
\sum_{j+m \leq k} \iint_{\mathscr{R}\left(\tau^{\prime}, \tau\right) \cap\left\{r \leq r_{Y}^{-}\right\}} K^{N}\left(\partial_{t^{*}}^{j} \hat{Y}^{m} \Phi\right) & \\
& \leq C \tau^{-2+\eta}\left(\sum_{j+m \leq k} \int_{\Sigma_{\tau_{0}}} J_{\mu}^{N}\left(\partial_{t^{*}}^{j} \hat{Y}^{m} \Phi\right) n_{\Sigma_{\tau_{0}}}^{\mu}+\sum_{j=0}^{k+2} \int_{\Sigma_{\tau_{0}}} J_{\mu}^{Z+C N}\left(\partial_{t^{*}}^{j} \Phi\right) n_{\Sigma_{\tau_{0}}}^{\mu}\right) .
\end{aligned}
$$

\section{Estimates for $\tilde{\Omega} \Phi$}

In this section, we would like to prove estimates for $\widetilde{\Omega}^{\ell} \Phi$. The estimates for $\widetilde{\Omega} \Phi$ are useful to provide an extra factor of $r$ in the energy estimates.

Proposition 49. We have

$$
\begin{aligned}
& \int_{\Sigma_{\tau} \cap\left\{r \geq r_{Y}^{-}\right\}} r^{2}\left|\not{ }^{2} \Phi\right|^{2} \leq C \int_{\Sigma_{\tau}} J_{\mu}^{N}\left(\Phi, \partial_{t^{*}} \Phi, \widetilde{\Omega} \Phi\right) n_{\Sigma_{\tau}}^{\mu}, \\
& \iint_{\mathscr{R}\left(\tau^{\prime}, \tau\right) \cap\left\{r \geq r_{Y}^{-}\right\}} r^{1-\delta}\left|\not^{2} \Phi\right|^{2} \leq C \iint_{\mathscr{R}\left(\tau^{\prime}, \tau\right)} r^{-1-\delta} J_{\mu}^{N}\left(\Phi, \partial_{t^{*}} \Phi, \widetilde{\Omega} \Phi\right) n_{\Sigma_{t^{*}}}^{\mu}, \\
& \iint_{\mathscr{R}\left(\tau^{\prime}, \tau\right) \cap\left\{r \geq r_{Y}^{-}\right\}} r^{1-\delta}\left|\not^{2} \Phi\right|^{2} \leq C \iint_{\mathscr{R}\left(\tau^{\prime}, \tau\right)} K^{X_{1}}\left(\Phi, \partial_{t^{*}} \Phi\right)+K^{X_{0}}(\widetilde{\Omega} \Phi) .
\end{aligned}
$$

To prove such estimates, we commute $\square_{g_{K}}$ with $\widetilde{\Omega}$. Recall from Section 4.3 that

$$
\left|\left[\square_{g_{K}}, \widetilde{\Omega}\right] \Phi\right| \leq C r^{-2}\left(\left|D^{2} \Phi\right|+|D \Phi|\right)
$$

everywhere, and

$$
\left[\square_{g_{K}}, \widetilde{\Omega}\right] \Phi=0
$$

for $r<R_{\Omega}$. Now suppose $\square_{g_{K}} \Phi=0$. We have

$$
\square_{g_{K}}\left(\widetilde{\Omega}^{\ell} \Phi\right)=\sum_{j=0}^{\ell-1} \widetilde{\Omega}^{j}\left[\square_{g_{K}}, \widetilde{\Omega}\right] \widetilde{\Omega}^{\ell-j-1} \Phi=: G_{\Omega, \ell}
$$

Since $[D, \widetilde{\Omega}]=D$, we have

$$
\left|G_{\Omega, \ell}\right| \leq C r^{-2}\left(\sum_{j=0}^{\ell-1}\left(\left|D^{2} \widetilde{\Omega}^{j} \Phi\right|+\left|D \widetilde{\Omega}^{j} \Phi\right|\right)+\sum_{j=0}^{\ell+1}\left|D^{j} \Phi\right|\right)
$$

and $G_{\Omega, \ell}$ is supported in $\left\{r \geq R_{\Omega}\right\}$.

Definition 50. $\quad E_{\Omega, \ell}=\iint_{\Re\left(\tau^{\prime}-1, \tau+1\right)} r^{1+\delta} G_{\Omega, \ell}^{2}+\sup _{t^{*} \in\left[\tau^{\prime}-1, \tau+1\right]} \int_{\Sigma_{t^{*}} \cap\left\{|r-3 M| \leq \frac{1}{8} M\right\}} G_{\Omega, \ell^{2}}$.

This is the error term for the energy estimates for $\widetilde{\Omega}^{\ell} \Phi$. We show that this can be controlled. 
Proposition 51. We have

$$
E_{\Omega, \ell} \leq C \sum_{m=0}^{1} \sum_{j=0}^{\ell-1} \iint_{\mathscr{R}\left(\tau^{\prime}-1, \tau+1\right) \cap\left\{r \geq R_{\Omega}\right\}} K^{X_{0}}\left(\partial_{t^{*}}^{m} \widetilde{\Omega}^{j} \Phi\right)+C \sum_{m=0}^{\ell} \iint_{\mathscr{R}\left(\tau^{\prime}-1, \tau+1\right) \cap\left\{r \geq R_{\Omega}\right\}} K^{X_{0}}\left(\partial_{t^{*}}^{m} \Phi\right) .
$$

Proof.

$$
\begin{aligned}
& \iint_{\Re\left(\tau^{\prime}-1, \tau+1\right)} r^{1+\delta} G_{\Omega, \ell}^{2} \\
& \leq C \sum_{j=0}^{\ell-1} \iint_{\Re\left(\tau^{\prime}-1, \tau+1\right) \cap\left\{r \geq R_{\Omega}\right\}} r^{-3+\delta}\left(\left(D^{2} \widetilde{\Omega}^{j} \Phi\right)^{2}+\left(D \widetilde{\Omega}^{j} \Phi\right)^{2}\right)+C \sum_{j=0}^{\ell+1} \iint_{\Re\left(\tau^{\prime}-1, \tau+1\right) \cap\left\{r \geq R_{\Omega}\right\}} r^{-3+\delta}\left(D^{j} \Phi\right)^{2} \\
& \leq C \sum_{m=0}^{1} \sum_{j=0}^{\ell-1} \iint_{\Re\left(\tau^{\prime}-1, \tau+1\right) \cap\left\{r \geq R_{\Omega}\right\}} r^{-3+\delta} J_{\mu}^{N}\left(\partial_{t^{*}}^{m} \widetilde{\Omega}^{j} \Phi\right) n_{\Sigma t^{*}}^{\mu}+C \sum_{m=0}^{\ell} r_{\mathscr{R}\left(\tau^{\prime}-1, \tau+1\right) \cap\left\{r \geq R_{\Omega}\right\}} r^{-3+\delta} J_{\mu}^{N}\left(\partial_{t^{*}}^{m} \Phi\right) n_{\Sigma t^{*}}^{\mu} \\
& \leq C \sum_{m=0}^{\ell} \sum_{j=0}^{\ell-1} \iint_{\Re\left(\tau^{\prime}-1, \tau+1\right) \cap\left\{r \geq R_{\Omega}\right\}} K^{X_{0}}\left(\partial_{t^{*}}^{m} \widetilde{\Omega}^{j} \Phi\right)+C \sum_{m=0}^{\ell} \iint_{\Re\left(\tau^{\prime}-1, \tau+1\right) \cap\left\{r \geq R_{\Omega}\right\}} K^{X_{0}\left(\partial_{t^{*}}^{m} \Phi\right) .}
\end{aligned}
$$

By choosing $R_{\Omega}$ sufficiently large, the second term of $E_{\Omega, \ell}$ vanishes.

We can show that the nondegenerate energy of $\widetilde{\Omega}^{\ell} \Phi$ is almost bounded.

Proposition 52. Suppose $\square_{g_{K}} \Phi=0$. Then

$$
\begin{array}{r}
\int_{\Sigma_{\tau}} J_{\mu}^{N}\left(\widetilde{\Omega}^{\ell} \Phi\right) n_{\Sigma_{\tau}}^{\mu}+\int_{\mathscr{H}\left(\tau_{0}, \tau\right)} J_{\mu}^{N}\left(\widetilde{\Omega}^{\ell} \Phi\right) n_{\mathscr{H}^{+}}^{\mu}+\iint_{\mathscr{R}\left(\tau_{0}, \tau\right) \cap\left\{r \leq r_{Y}^{-}\right\}} K^{N}\left(\widetilde{\Omega}^{\ell} \Phi\right)+\iint_{\mathscr{R}\left(\tau_{0}, \tau\right)} K^{X_{0}}\left(\widetilde{\Omega}^{\ell} \Phi\right) \\
\leq C \sum_{i+j \leq \ell} \int_{\Sigma_{\tau_{0}}} J_{\mu}^{N}\left(\partial_{t^{*}}^{i} \widetilde{\Omega}^{j} \Phi\right) n_{\Sigma_{\tau_{0}}}^{\mu}
\end{array}
$$

Proof. We prove this by induction on $\ell$. The $\ell=0$ case is true by setting $G=0$ in Proposition 28 . We assume that the proposition is true for $\ell \leq \ell_{0}-1$. This in particular implies, after a commutations with the Killing vector field $\partial_{t^{*}}$, that

$$
\sum_{j=0}^{\ell_{0}-1} \iint_{\mathscr{R}\left(\tau_{0}, \tau\right)} K^{X_{0}}\left(\partial_{t^{*}}^{m} \widetilde{\Omega}^{j} \Phi\right) \leq C \sum_{i+j \leq m+\ell_{0}-1} \int_{\Sigma_{\tau_{0}}} J_{\mu}^{N}\left(\partial_{t^{*}}^{i} \widetilde{\Omega}^{j} \Phi\right) n_{\Sigma_{\tau_{0}}}^{\mu}
$$


By Propositions 27 and 51,

$$
\begin{aligned}
& \int_{\Sigma_{\tau}} J_{\mu}^{N}\left(\widetilde{\Omega}^{\ell_{0}} \Phi\right) n_{\Sigma_{\tau}}^{\mu}+\int_{\mathscr{H}\left(\tau_{0}, \tau\right)} J_{\mu}^{N}\left(\widetilde{\Omega}^{\ell_{0}} \Phi\right) n_{\mathscr{H}^{+}}^{\mu}+\iint_{\mathscr{R}\left(\tau_{0}, \tau\right) \cap\left\{r \leq r_{Y}^{-}\right\}} K^{N}\left(\widetilde{\Omega}^{\ell_{0}} \Phi\right)+\iint_{\mathscr{R}\left(\tau_{0}, \tau\right)} K^{X_{0}}\left(\widetilde{\Omega}^{\ell_{0}} \Phi\right) \\
& \leq C\left(\int_{\Sigma_{\tau_{0}}} J_{\mu}^{N}\left(\widetilde{\Omega}^{\ell_{0}} \Phi\right) n_{\Sigma_{\tau_{0}}}^{\mu}+\iint_{\mathscr{R}\left(\tau^{\prime}-1, \tau+1\right)} r^{1+\delta} G_{\Omega, \ell_{0}}^{2}+\sup _{t^{*} \in\left[\tau^{\prime}-1, \tau+1\right]} \int_{\Sigma_{t^{*}} \cap\left\{|r-3 M| \leq \frac{1}{8} M\right\}} G_{\Omega, \ell_{0}}^{2}\right) \\
& \leq C\left(\int_{\Sigma_{\tau_{0}}} J_{\mu}^{N}\left(\widetilde{\Omega}^{\ell_{0}} \Phi\right) n_{\Sigma_{\tau_{0}}}^{\mu}+C \sum_{m=0}^{1} \sum_{j=0}^{\ell_{0}-1} \iint_{\mathscr{R}\left(\tau_{0}-1, \tau+1\right) \cap\left\{r \geq R_{\Omega}\right\}} K^{X_{0}}\left(\partial_{t^{*}}^{m} \widetilde{\Omega}^{j} \Phi\right)\right. \\
& \left.+C \sum_{m=0}^{\ell_{0}} \iint_{\mathscr{R}\left(\tau_{0}-1, \tau+1\right) \cap\left\{r \geq R_{\Omega}\right\}} K^{X_{0}}\left(\partial_{t^{*}}^{m} \Phi\right)\right) \\
& \leq C \sum_{i+j \leq \ell_{0}} \int_{\Sigma_{\tau_{0}}} J_{\mu}^{N}\left(\partial_{t^{*}}^{i} \widetilde{\Omega}^{j} \Phi\right) n_{\Sigma_{\tau_{0}}}^{\mu}
\end{aligned}
$$

Remark. Only the $\ell=1$ case will be used.

\section{Estimates for $S \Phi$}

We will now use the energy estimates that we have derived to control $S \Phi$. In particular, we would like to prove a local integrated decay estimate for $S \Phi$. This will be used in the next section where we prove our main theorem. Recall from Section 4.2 that for $r$ large

$$
\begin{array}{r}
\left|\left[\square_{g_{K}}, S\right] \Phi-\left(2+\frac{r^{*} \mu}{r}\right) \square_{g_{K}} \Phi-\frac{2}{r}\left(\frac{r^{*}}{r}-1-\frac{2 r^{*} \mu}{r}\right) \partial_{r^{*}} \Phi-2\left(\left(\frac{r^{*}}{r}-1\right)-\frac{3 r^{*} \mu}{2 r}\right) \Delta \Phi\right| \\
\leq C \epsilon r^{-2}\left(\sum_{k=1}^{2}\left|\partial^{k} \Phi\right|\right),
\end{array}
$$

and that for $r \leq R$, we have

$$
\left|\left[\square_{g_{K}}, S\right] \Phi\right| \leq C\left(\sum_{k=1}^{2}\left|D^{k} \Phi\right|\right) .
$$

From now on we will prove estimates for $S \Phi$ by considering the wave equation that it satisfies. We will assume, as before, $\square_{g_{K}} \Phi=0$ and let $G$ denote the commutator term, that is, $\square_{g_{K}}(S \Phi)=G$. If we look at our estimates in the previous sections, we will need to control $G$ in three different norms. We now consider them separately.

Proposition 53. Let $\tau^{\prime} \leq \tau \leq(1.1) \tau^{\prime}$. Then

$$
\begin{aligned}
& \sum_{m=0}^{\ell} \iint_{\mathscr{R}\left(\tau^{\prime}-1, \tau+1\right)} r^{1+\delta}\left(\partial_{t^{*}}^{m} G\right)^{2} \\
& \leq C \tau^{-1+\eta} \sum_{m+k+j \leq \ell+3}\left(\int_{\Sigma_{\tau_{0}}} J_{\mu}^{Z, w^{Z}}\left(\partial_{t^{*}}^{m} \hat{Y}^{k} \widetilde{\Omega}^{j} \Phi\right) n_{\Sigma_{\tau_{0}}}^{\mu}+C \int_{\Sigma_{\tau_{0}}} J_{\mu}^{N}\left(\partial_{t^{*}}^{m} \hat{Y}^{k} \widetilde{\Omega}^{j} \Phi\right) n_{\Sigma_{\tau_{0}}}^{\mu}\right) .
\end{aligned}
$$


and

$$
\begin{aligned}
\sum_{m=0}^{\ell} \iint_{\mathscr{R}\left(\tau^{\prime}-1, \tau+1\right) \cap\left\{r \leq \frac{9}{10} t^{*}\right\}} r^{1+\delta}\left(\partial_{t^{*}}^{m} G\right)^{2} & \leq C \tau^{-2+\eta} \sum_{m+k+j \leq \ell+4}\left(\int_{\Sigma_{\tau_{0}}} J_{\mu}^{Z, w^{Z}}\left(\partial_{t^{*}}^{m} \hat{Y}^{k} \widetilde{\Omega}^{j} \Phi\right) n_{\Sigma_{\tau_{0}}}^{\mu}+C \int_{\Sigma_{\tau_{0}}} J_{\mu}^{N}\left(\partial_{t^{*}}^{m} \hat{Y}^{k} \widetilde{\Omega}^{j} \Phi\right) n_{\Sigma_{\tau_{0}}}^{\mu}\right) .
\end{aligned}
$$

In other words, we can get more decay if we localize and allow an extra derivative.

Proof.

$$
\begin{aligned}
& \sum_{m=0}^{\ell} \iint_{\Re\left(\tau^{\prime}-1, \tau+1\right)} r^{1+\delta}\left(\partial_{t^{*}}^{m} G\right)^{2} \\
& \leq C \sum_{m=0}^{\ell} \iint_{\mathscr{R}\left(\tau^{\prime}-1, \tau+1\right)} r^{-3+\delta}\left(\left(\partial_{t^{*}}^{m} D^{2} \Phi\right)^{2}+\left(\partial_{t^{*}}^{m} D \Phi\right)^{2}+\left(r \partial_{t^{*}}^{m} \Delta \Phi\right)^{2}\right) \\
& \text { (noting that the } \delta \text { in the two lines are different) } \\
& \leq C \sum_{m+k \leq \ell+1}\left(\iint_{\mathscr{R}\left(\tau^{\prime}-1, \tau+1\right) \cap\left\{r \leq \frac{1}{2} t^{*}\right\}} r^{-1+\delta} J_{\mu}^{N}\left(\partial_{t^{*}}^{m} \hat{Y}^{k} \Phi\right) n_{\Sigma_{t}^{*}}^{\mu}\right. \\
& \left.+\iint_{\mathscr{R}\left(\tau^{\prime}-1, \tau+1\right) \cap\left\{r \geq \frac{1}{2} t^{*}\right\}} r^{-3+\delta} J_{\mu}^{N}\left(\partial_{t^{*}}^{m} \widetilde{\Omega}^{k} \Phi\right) n_{\Sigma_{t}^{*}}^{\mu}\right) \\
& \text { (by Proposition 44, } 45 \text { and 49) } \\
& \leq C \sum_{m+k \leq \ell+3} \tau^{-1+\eta}\left(\int_{\Sigma_{\tau_{0}}} J_{\mu}^{Z, w^{Z}}\left(\partial_{t^{*}}^{m} \Phi\right) n_{\Sigma_{\tau_{0}}}^{\mu}+C \int_{\Sigma_{\tau_{0}}} J_{\mu}^{N}\left(\partial_{t^{*}}^{m} \hat{Y}^{k} \Phi\right) n_{\Sigma_{\tau_{0}}}^{\mu}\right) \\
& +C \sum_{m+k \leq \ell+3} \int_{\tau^{\prime}-1}^{\tau+1}\left(t^{*}\right)^{-3+\delta}\left(\int_{\Sigma_{\tau_{0}}} J_{\mu}^{Z, w^{Z}}\left(\partial_{t^{*}}^{m} \Phi\right) n_{\Sigma_{\tau_{0}}}^{\mu}+C \int_{\Sigma_{\tau_{0}}} J_{\mu}^{N}\left(\partial_{t^{*}}^{m} \hat{Y}^{k} \Phi\right) n_{\Sigma_{\tau_{0}}}^{\mu}\right) d t^{*} \\
& +C \sum_{m \leq \ell+1} \int_{\tau^{\prime}-1}^{\tau+1}\left(t^{*}\right)^{-3+\delta}\left(\int_{\Sigma_{\tau_{0}}} J_{\mu}^{N}\left(\partial_{t^{*}}^{m} \widetilde{\Omega} \Phi\right) n_{\Sigma_{\tau_{0}}}^{\mu}\right) d t^{*} \\
& \text { (using Corollaries 43, } 48 \text { and Proposition 52) } \\
& \leq C \tau^{-1+\eta} \sum_{m+k+j \leq \ell+3}\left(\int_{\Sigma_{\tau_{0}}} J_{\mu}^{Z, w^{Z}}\left(\partial_{t^{*}}^{m} \hat{Y}^{k} \widetilde{\Omega}^{j} \Phi\right) n_{\Sigma_{\tau_{0}}}^{\mu}+C \int_{\Sigma_{\tau_{0}}} J_{\mu}^{N}\left(\partial_{t^{*}}^{m} \hat{Y}^{k} \widetilde{\Omega}^{j} \Phi\right) n_{\Sigma_{\tau_{0}}}^{\mu}\right) .
\end{aligned}
$$

We then move on to the localized version:

$$
\begin{aligned}
& \sum_{m=0}^{\ell} \iint_{\mathscr{R}\left(\tau^{\prime}-1, \tau+1\right) \cap\left\{r \leq \frac{9}{10} t^{*}\right\}} r^{1+\delta}\left(\partial_{t^{*}}^{m} G\right)^{2} \\
& \leq C \sum_{m=0}^{\ell} \iint_{\mathscr{R}\left(\tau^{\prime}-1, \tau+1\right) \cap\left\{r \leq \frac{9}{10} t^{*}\right\}} r^{-3+\delta}\left(\left(\partial_{t^{*}}^{m} D^{2} \Phi\right)^{2}+\left(\partial_{t^{*}}^{m} D \Phi\right)^{2}+\left(r \partial_{t^{*}}^{m} \Delta \Phi\right)^{2}\right)
\end{aligned}
$$




$$
\begin{aligned}
& \leq C \sum_{m+k \leq \ell+1}\left(\iint_{\mathscr{R}\left(\tau^{\prime}-1, \tau+1\right) \cap\left\{r \leq \frac{1}{2} t^{*}\right\}} r^{-1+\delta} J_{\mu}^{N}\left(\partial_{t^{*}}^{m} \hat{Y} \Phi\right) n_{\Sigma_{t}^{*}}^{\mu}+\int_{\mathscr{R}\left(\tau^{\prime}-1, \tau+1\right) \cap\left\{\frac{1}{2} t^{*} \leq r \leq \frac{19 t^{*}}{20}\right\}} r^{-3+\delta} J_{\mu}^{N}\left(\partial_{t^{*}}^{m} \hat{Y} \Phi\right) n_{\Sigma_{t}^{*}}^{\mu}\right) \\
& +C \sum_{m=0}^{1}\left(\iint_{\mathscr{R}\left(\tau^{\prime}-1, \tau+1\right) \cap\left\{\frac{1}{2} t^{*} \leq r \leq \frac{19 t^{*}}{20}\right\}} r^{-3+\delta} J_{\mu}^{N}\left(\partial_{t^{*}}^{m} \widetilde{\Omega} \Phi\right) n_{\Sigma_{t}^{*}}^{\mu}\right) \quad \text { (by Proposition 44, } 45 \text { and 49) } \\
& \leq C \sum_{m+k \leq \ell+4} \tau^{-2+\eta}\left(\int_{\Sigma_{\tau_{0}}} J_{\mu}^{Z, w^{Z}}\left(\partial_{t^{*}}^{m} \Phi\right) n_{\Sigma_{\tau_{0}}}^{\mu}+C \int_{\Sigma_{\tau_{0}}} J_{\mu}^{N}\left(\partial_{t^{*}}^{m} \hat{Y} \Phi\right) n_{\Sigma_{\tau_{0}}}^{\mu}\right) \\
& +C \sum_{m+k \leq \ell+3} \int_{\tau^{\prime}-1}^{\tau+1}\left(t^{*}\right)^{-3+\delta}\left(\int_{\Sigma_{\tau_{0}}} J_{\mu}^{Z, w^{Z}}\left(\partial_{t^{*}}^{m} \Phi\right) n_{\Sigma_{\tau_{0}}}^{\mu}+C \int_{\Sigma_{\tau_{0}}} J_{\mu}^{N}\left(\partial_{t^{*}}^{m} \hat{Y} \Phi\right) n_{\Sigma_{\tau_{0}}}^{\mu}\right) d t^{*} \\
& +C \sum_{m=0}^{\ell} \int_{\tau^{\prime}-1}^{\tau+1}\left(t^{*}\right)^{-3+\delta}\left(\int_{\Sigma_{\tau_{0}}} J_{\mu}^{N}\left(\partial_{t^{*}}^{m} \widetilde{\Omega} \Phi\right) n_{\Sigma_{\tau_{0}}}^{\mu}\right) d t^{*} \quad \text { (using Corollaries 43, } 48 \text { and Proposition 52) } \\
& \leq C \tau^{-2+\eta} \sum_{m+k+j \leq \ell+4}\left(\int_{\Sigma_{\tau_{0}}} J_{\mu}^{Z, w^{Z}}\left(\partial_{t^{*}}^{m} \hat{Y}^{k} \widetilde{\Omega}^{j} \Phi\right) n_{\Sigma_{\tau_{0}}}^{\mu}+C \int_{\Sigma_{\tau_{0}}} J_{\mu}^{N}\left(\partial_{t^{*}}^{m} \hat{Y}^{k} \widetilde{\Omega}^{j} \Phi\right) n_{\Sigma_{\tau_{0}}}^{\mu}\right) .
\end{aligned}
$$

To estimate the inhomogeneous term in the region $r \leq \frac{1}{2} t^{*}$, we will also need to estimate a term not integrated over $t^{*}$, which arises from the integration by parts.

Proposition 54. For $\tau^{\prime} \leq \tau \leq(1.1) \tau^{\prime}$,

$$
\begin{aligned}
\sup _{t^{*} \in\left[\tau^{\prime}-1, \tau+1\right]} \sum_{m=0}^{\ell} \int_{\Sigma_{t^{*}} \cap\left\{|r-3 M| \leq \frac{1}{8} M\right\}}\left(\partial_{t^{*}}^{m} G\right)^{2} & \leq C \tau^{-2+\eta} \sum_{m+j \leq \ell+3}\left(\int_{\Sigma_{\tau_{0}}} J_{\mu}^{Z, w^{Z}}\left(\partial_{t^{*}}^{m} \widetilde{\Omega}^{j} \Phi\right) n_{\Sigma_{\tau_{0}}}^{\mu}+C \int_{\Sigma_{\tau_{0}}} J_{\mu}^{N}\left(\partial_{t^{*}}^{m} \widetilde{\Omega}^{j} \Phi\right) n_{\Sigma_{\tau_{0}}}^{\mu}\right) .
\end{aligned}
$$

Proof.

$$
\begin{aligned}
& \sup _{t^{*} \in\left[\tau^{\prime}-1, \tau+1\right]} \sum_{m=0}^{\ell} \int_{\Sigma_{t^{*}} \cap\left\{|r-3 M| \leq \frac{1}{8} M\right\}}\left(\partial_{t^{*}}^{m} G\right)^{2} \\
& \leq C \sup _{t^{*} \in\left[\tau^{\prime}-1, \tau+1\right]} \sum_{m=0}^{\ell} \int_{\Sigma_{t^{*}} \cap\left\{r_{Y}^{-} \leq r \leq \frac{25}{8} M\right\}}\left(\left(D^{2} \partial_{t^{*}}^{m} \Phi\right)^{2}+\left(D \partial_{t^{*}}^{m} \Phi\right)^{2}+\left(r \Delta \partial_{t^{*}}^{m} \Phi\right)^{2}\right) \\
& \leq C \sup _{t^{*} \in\left[\tau^{\prime}-1, \tau+1\right]}\left(\sum_{m=0}^{\ell+1} \int_{\Sigma_{t^{*}} \cap\left\{r_{Y}^{-} \leq r \leq \frac{25}{8} M\right\}} J_{\mu}^{N}\left(\partial_{t^{*}}^{m} \Phi\right) n_{\Sigma_{t}^{*}}^{\mu}+\sum_{m=0}^{\ell} \int_{\Sigma_{t^{*} \cap\left\{r_{Y}^{-} \leq r \leq \frac{25}{8} M\right\}}} J_{\mu}^{N}\left(\widetilde{\Omega} \partial_{t^{*}}^{m} \Phi\right) n_{\Sigma_{t}^{*}}^{\mu}\right)
\end{aligned}
$$

(by Proposition 44 and 49)$$
\leq C \tau^{-2+\eta} \sum_{m+j \leq \ell+3}\left(\int_{\Sigma_{\tau_{0}}} J_{\mu}^{Z, w^{Z}}\left(\partial_{t^{*}}^{m} \widetilde{\Omega}^{j} \Phi\right) n_{\Sigma_{\tau_{0}}}^{\mu}+C \int_{\Sigma_{\tau_{0}}} J_{\mu}^{N}\left(\partial_{t^{*}}^{m} \widetilde{\Omega}^{j} \Phi\right) n_{\Sigma_{\tau_{0}}}^{\mu}\right),
$$

(using Corollary 43 and Proposition 52). 
Finally, we estimate the third norm:

Proposition 55. $\sum_{m=0}^{\ell}\left(\int_{\tau_{0}}^{\tau}\left(\int_{\Sigma_{t^{*}} \cap\left\{r \geq \frac{1}{2} t^{*}\right\}} r^{2}\left(\partial_{t^{*}}^{m} G\right)^{2}\right)^{1 / 2} d t^{*}\right)^{2} \leq C \tau^{\eta} \sum_{m+j \leq \ell+3} \int_{\Sigma_{\tau_{0}}} J_{\mu}^{N}\left(\partial_{t^{*}}^{m} \widetilde{\Omega}^{j} \Phi\right) n_{\Sigma_{\tau_{0}}}^{\mu}$.

Proof.

$$
\begin{aligned}
& \sum_{m=0}^{\ell}\left(\int_{\tau_{0}}^{\tau}\left(\int_{\Sigma_{t^{*}} \cap\left\{r \geq \frac{1}{2} t^{*}\right\}} r^{2}\left(\partial_{t^{*}}^{m} G\right)^{2}\right)^{1 / 2} d t^{*}\right)^{2} \\
& \quad \leq C \sum_{m=0}^{\ell}\left(\int_{\tau_{0}}^{\tau}\left(t^{*}\right)^{-1+\delta}\left(\int_{\Sigma_{t^{*}} \cap\left\{r \geq \frac{1}{2} t^{*}\right\}}\left(\left(D^{2} \Phi\right)^{2}+\left(D \partial_{t^{*}}^{m} \Phi\right)^{2}+\left(r \phi \partial_{t^{*}}^{m} \Phi\right)^{2}\right)\right)^{1 / 2} d t^{*}\right)^{2} \\
& \quad \leq C\left(\int_{\tau_{0}}^{\tau}\left(t^{*}\right)^{-1+\delta}\left(\sum_{m=0}^{\ell+1} \int_{\Sigma_{t^{*}}} J_{\mu}^{N}\left(\partial_{t^{*}}^{m} \Phi\right) n_{\Sigma_{t^{*}}}^{\mu}+\sum_{m=0}^{\ell} \int_{\Sigma_{t^{*}}} J_{\mu}^{N}\left(\widetilde{\Omega} \partial_{t^{*}}^{m} \Phi\right) n_{\Sigma_{t^{*}}}^{\mu}\right)^{1 / 2} d t^{*}\right)^{2} \\
& \quad \leq C \tau^{\eta} \sum_{m+j \leq \ell+3} \int_{\Sigma_{\tau_{0}}} J_{\mu}^{N}\left(\partial_{t^{*}}^{m} \widetilde{\Omega}^{j} \Phi\right) n_{\Sigma_{\tau_{0}}}^{\mu} .
\end{aligned}
$$

Now that we have control of the inhomogeneous terms in the equation $\square_{g_{K}} \Phi=G$, we can prove the decay of $S \Phi$. To this end, we will introduce the bootstrap assumptions:

$$
\begin{aligned}
& c \int_{\Sigma_{\tau}} J_{\mu}^{Z, w^{Z}}\left(\partial_{t^{*}} S \Phi\right) n_{\Sigma_{\tau}}^{\mu}+\tau^{2} \int_{\Sigma_{\tau} \cap\{r \leq \gamma \tau\}} J_{\mu}^{N}\left(\partial_{t^{*}} S \Phi\right) n_{\Sigma_{\tau}}^{\mu} \\
& \leq A \tau \sum_{m=1}^{2} \int_{\Sigma_{\tau_{0}}} J_{\mu}^{Z+C N, w^{Z}}\left(\partial_{t^{*}}^{m} S \Phi\right) n_{\Sigma_{\tau_{0}}}^{\mu}+A \tau^{1+\eta} \sum_{m+k+j \leq 5} \int_{\Sigma_{\tau_{0}}} J_{\mu}^{Z+C N, w^{Z}}\left(\partial_{t^{*}}^{m} \hat{Y}^{k} \widetilde{\Omega}^{j} \Phi\right) n_{\Sigma_{\tau_{0}}}^{\mu}, \\
& c \int_{\Sigma_{\tau}} J_{\mu}^{Z, w^{Z}}(S \Phi) n_{\Sigma_{\tau}}^{\mu}+\tau^{2} \int_{\Sigma_{\tau} \cap\{r \leq \gamma \tau\}} J_{\mu}^{N}(S \Phi) n_{\Sigma_{\tau}}^{\mu} \\
& \leq A^{2} \tau^{\eta}\left(\sum_{m=0}^{2} \int_{\Sigma_{\tau_{0}}} J_{\mu}^{Z+C N, w^{Z}}\left(\partial_{t^{*}}^{m} S \Phi\right) n_{\Sigma_{\tau_{0}}}^{\mu}+\sum_{m+k+j \leq 5} \int_{\Sigma_{\tau_{0}}} J_{\mu}^{Z+C N, w^{Z}}\left(\partial_{t^{*}}^{m} \hat{Y}^{k} \widetilde{\Omega}^{j} \Phi\right) n_{\Sigma_{\tau_{0}}}^{\mu}\right) .
\end{aligned}
$$

We think of $A$ as some large constant to be chosen. We will improve the constants $A$ and $A^{2}$ in the assumptions above. Under these two assumptions, we will get the following three estimates for the bulk terms:

\section{Proposition 56.}

$$
\iint_{\mathscr{R}\left(\tau_{0}, \tau\right)} K^{X_{1}}\left(\partial_{t^{*}} S \Phi\right) \leq C\left(\sum_{m=1}^{2} \int_{\Sigma_{\tau_{0}}} J_{\mu}^{N}\left(\partial_{t^{*}}^{m} S \Phi\right) n_{\Sigma_{\tau_{0}}}^{\mu}+\sum_{m+k+j \leq 5} \int_{\Sigma_{\tau_{0}}} J_{\mu}^{Z+C N, w^{Z}}\left(\partial_{t^{*}}^{m} \hat{Y} \widetilde{\Omega}^{j} \Phi\right) n_{\Sigma_{\tau_{0}}}^{\mu}\right) .
$$

Proof. The follows by Proposition 29 applied to the equation $\square_{g_{K}}\left(\partial_{t^{*}} S \Phi\right)=\partial_{t^{*}} G$, taking $\tau^{\prime}=\tau_{0}, G_{1}=0$, and $G_{2}=\partial_{t^{*}} G$. Then use Propositions 53 and 54 to estimate the terms with $G$. 
Proposition 57. For $\tau^{\prime} \leq \tau \leq(1.1) \tau^{\prime}$, we have

$$
\begin{aligned}
\iint_{\mathscr{R}\left(\tau^{\prime}, \tau\right) \cap\left\{r \leq r_{Y}^{-}\right\}} K^{N}\left(\partial_{t^{*}} S \Phi\right)+\iint_{\mathscr{R}\left(\tau^{\prime}, \tau\right) \cap\left\{r \leq \frac{1}{2} t^{*}\right\}} K^{X_{0}}\left(\partial_{t^{*}} S \Phi\right)+\iint_{\mathscr{R}\left(\tau_{0}, \tau\right)} r^{-1+\delta} K^{X_{1}}(S \Phi) \\
\leq C A\left(\tau^{-2} \int_{\Sigma_{\tau^{\prime}}} J_{\mu}^{Z, w^{Z}}(S \Phi) n_{\Sigma_{\tau^{\prime}}}^{\mu}+C \int_{\Sigma_{\tau^{\prime}} \cap\left\{r \leq r_{Y}^{-}\right\}} J_{\mu}^{N}(S \Phi) n_{\Sigma_{\tau^{\prime}}}^{\mu}\right) \\
+C A \tau^{-1+\eta} \sum_{m+k+j \leq 5} \int_{\Sigma_{\tau_{0}}} J_{\mu}^{Z+C N, w^{Z}}\left(\partial_{t^{*}}^{m} \hat{Y} \widetilde{\Omega}^{j} \Phi\right) n_{\Sigma_{\tau_{0}}}^{\mu} .
\end{aligned}
$$

Proof. By Propositions 33 and 34, taking $G_{1}=0$ and $G_{2}=G$, and using Propositions 53 and 54 to estimate the terms with $G$, we have

$$
\begin{array}{r}
\iint_{\mathscr{R}\left(\tau^{\prime}, \tau\right) \cap\left\{r \leq r_{Y}^{-}\right\}} K^{N}\left(\partial_{t^{*}} S \Phi\right)+\iint_{\mathscr{R}\left(\tau^{\prime}, \tau\right) \cap\left\{r \leq \frac{1}{2} t^{*}\right\}} K^{X_{0}}\left(\partial_{t^{*}} S \Phi\right)+\iint_{\mathscr{R}\left(\tau_{0}, \tau\right) \cap\left\{r \leq \frac{1}{2} t^{*}\right\}} K^{X_{1}}(S \Phi) \\
\leq C A\left(\tau^{-2} \int_{\Sigma_{\tau^{\prime}}} J_{\mu}^{Z, w^{Z}}(S \Phi) n_{\Sigma_{\tau^{\prime}}}^{\mu}+C \int_{\Sigma_{\tau^{\prime}} \cap\left\{r \leq r_{Y}^{-}\right\}} J_{\mu}^{N}(S \Phi) n_{\Sigma_{\tau^{\prime}}}^{\mu}\right) \\
+C A \tau^{-1+\eta} \sum_{m+k+j \leq 5} \int_{\Sigma_{\tau_{0}}} J_{\mu}^{Z+C N, w^{Z}}\left(\partial_{t^{*}}^{m} \hat{Y} \widetilde{\Omega}^{j} \Phi\right) n_{\Sigma_{\tau_{0}}}^{\mu} \cdot
\end{array}
$$

It remains to estimate $r^{-1+\delta} K^{X_{1}}$ in the region $r \geq \frac{1}{2} t^{*}$. Here, we will use crucially the decay in $r$. Clearly,

$$
\iint_{\mathscr{R}\left(\tau_{0}, \tau\right) \cap\left\{r \geq \frac{1}{2} t^{*}\right\}} r^{-1+\delta} K^{X_{1}}(S \Phi) \leq C \tau^{-1+\delta} \iint_{\mathscr{R}\left(\tau_{0}, \tau\right)} K^{X_{1}}(S \Phi) .
$$

Then we can estimate the right-hand side by Proposition 29, taking $\tau^{\prime}=\tau_{0}, \quad G_{1}=0$ and $G_{2}=\partial_{t^{*}} G$. Then use Propositions 53 and 54 to estimate the terms with $G$.

Proposition 58. For $\tau^{\prime} \leq \tau \leq(1.1) \tau^{\prime}$,

$$
\begin{aligned}
& \iint_{\mathscr{R}\left(\tau^{\prime}, \tau\right) \cap\left\{r \leq r_{Y}^{-}\right\}} K^{N}(S \Phi)+\iint_{\mathscr{R}\left(\tau^{\prime}, \tau\right) \cap\left\{r \leq \frac{1}{2} t^{*}\right\}} K^{X_{0}}(S \Phi) \\
& \leq C A^{2}\left(\tau^{-2} \int_{\Sigma_{\tau^{\prime}}} J_{\mu}^{Z, w^{Z}}(S \Phi) n_{\Sigma_{\tau^{\prime}}}^{\mu}+C \int_{\Sigma_{\tau^{\prime}} \cap\left\{r \leq r_{Y}^{-}\right\}} J_{\mu}^{N}(S \Phi) n_{\Sigma_{\tau^{\prime}}}^{\mu}\right) \\
& \quad+C A^{2} \tau^{-2+\eta} \sum_{m+k+j \leq 4} \int_{\Sigma_{\tau_{0}}} J_{\mu}^{Z+C N, w^{Z}}\left(\partial_{t^{*}}^{m} \hat{Y} \widetilde{\Omega}^{j} \Phi\right) n_{\Sigma_{\tau_{0}}}^{\mu} .
\end{aligned}
$$

Proof. This follows from using Proposition 33, taking $G_{1}=0$ and $G_{2}=G$, and using Propositions 53 and 54 to estimate the terms with $G$.

We are now ready to retrieve the bootstrap assumptions. First, we retrieve the assumption (23): 


\section{Proposition 59.}

$c \int_{\Sigma_{\tau}} J_{\mu}^{Z, w^{Z}}\left(\partial_{t^{*}} S \Phi\right) n_{\Sigma_{\tau}}^{\mu}+\tau^{2} \int_{\Sigma_{\tau} \cap\{r \leq \gamma \tau\}} J_{\mu}^{N}\left(\partial_{t^{*}} S \Phi\right) n_{\Sigma_{\tau}}^{\mu}$

$$
\leq \frac{1}{2} A \tau \sum_{m=1}^{2} \int_{\Sigma_{\tau_{0}}} J_{\mu}^{Z+C N, w^{Z}}\left(\partial_{t^{*}}^{m} S \Phi\right) n_{\Sigma_{\tau_{0}}}^{\mu}+\frac{1}{2} A \tau^{1+\eta} \sum_{m+k+j \leq 5} \int_{\Sigma_{\tau_{0}}} J_{\mu}^{Z+C N, w^{Z}}\left(\partial_{t^{*}}^{m} \hat{Y}^{k} \widetilde{\Omega}^{j} \Phi\right) n_{\Sigma_{\tau_{0}}}^{\mu} .
$$

Proof. By Proposition 41,

$$
\begin{aligned}
& c \int_{\Sigma_{\tau}} J_{\mu}^{Z, w^{Z}}\left(\partial_{t^{*}} S \Phi\right) n_{\Sigma_{\tau}}^{\mu}+\tau^{2} \int_{\Sigma_{\tau} \cap\{r \leq \gamma \tau\}} J_{\mu}^{N}\left(\partial_{t^{*}} S \Phi\right) n_{\Sigma_{\tau}}^{\mu} \\
& \leq C \int_{\Sigma_{\tau_{0}}} J_{\mu}^{Z+C N, w^{Z}}\left(\partial_{t^{*}} S \Phi\right) n_{\Sigma_{\tau_{0}}}^{\mu}+C \iint_{\mathscr{R}\left(\tau_{0}, \tau\right)} t^{*} r^{-1+\delta} K^{X_{1}}\left(\partial_{t^{*}} S \Phi\right) \\
& +C \delta^{\prime} \iint_{\mathscr{R}\left(\tau_{0}, \tau\right) \cap\left\{r \leq \frac{1}{2} t^{*}\right\}}\left(t^{*}\right)^{2} K^{X_{0}}\left(\partial_{t^{*}} S \Phi\right)+C\left(\delta^{\prime}+\epsilon\right) \iint_{\mathscr{R}\left(\tau_{0}, \tau\right) \cap\left\{r \leq r_{Y}^{-}\right\}}\left(t^{*}\right)^{2} K^{N}\left(\partial_{t^{*}} S \Phi\right) \\
& +C\left(\delta^{\prime}\right)^{-1}\left(\int_{\tau_{0}}^{\tau}\left(\int_{\Sigma_{t^{*}} \cap\left\{r \geq \frac{1}{2} t^{*}\right\}} r^{2}\left(\partial_{t^{*}} G\right)^{2}\right)^{1 / 2} d t^{*}\right)^{2}+C\left(\delta^{\prime}\right)^{-1} \sum_{m=1}^{2} \iint_{\mathscr{R}\left(\tau_{0}, \tau\right) \cap\left\{r \leq \frac{9}{10} t^{*}\right\}}\left(t^{*}\right)^{2} r^{1+\delta}\left(\partial_{t^{*}}^{m} G\right)^{2} \\
& +C\left(\delta^{\prime}\right)^{-1} \sup _{t^{*} \in\left[\tau_{0}, \tau\right]} \int_{\Sigma_{t^{*}} \cap\left\{r_{Y}^{-} \leq r \leq \frac{25}{8} M\right\}}\left(t^{*}\right)^{2}\left(\partial_{t^{*}} G\right)^{2} .
\end{aligned}
$$

It suffices to check that by Propositions 53, 54 and 55, all terms are acceptable.

We can now retrieve the bootstrap assumption (24).

\section{Proposition 60.}

$$
\begin{aligned}
c \int_{\Sigma_{\tau}} J_{\mu}^{Z, w^{Z}}(S \Phi) & n_{\Sigma_{\tau}}^{\mu}+\tau^{2} \int_{\Sigma_{\tau} \cap\{r \leq \gamma \tau\}} J_{\mu}^{N}(S \Phi) n_{\Sigma_{\tau}}^{\mu} \\
& \leq A^{2} \tau^{\eta}\left(\sum_{m=0}^{2} \int_{\Sigma_{\tau_{0}}} J_{\mu}^{Z+C N, w^{Z}}\left(\partial_{t^{*}}^{m} S \Phi\right) n_{\Sigma_{\tau_{0}}}^{\mu}+\sum_{m+k+j \leq 5} \int_{\Sigma_{\tau_{0}}} J_{\mu}^{Z+C N, w^{Z}}\left(\partial_{t^{*}}^{m} \hat{Y}^{k} \widetilde{\Omega}^{j} \Phi\right) n_{\Sigma_{\tau_{0}}}^{\mu}\right) .
\end{aligned}
$$


Proof. By Proposition 41,

$$
\begin{aligned}
& c \int_{\Sigma_{\tau}} J_{\mu}^{Z, w^{Z}}(S \Phi) n_{\Sigma_{\tau}}^{\mu}+\tau^{2} \int_{\Sigma_{\tau} \cap\{r \leq \gamma \tau\}} J_{\mu}^{N}(S \Phi) n_{\Sigma_{\tau}}^{\mu} \\
& \leq C \int_{\Sigma_{\tau_{0}}} J_{\mu}^{Z+C N, w^{Z}}(S \Phi) n_{\Sigma_{\tau_{0}}}^{\mu}+C \iint_{\mathscr{R}\left(\tau_{0}, \tau\right)} t^{*} r^{-1+\delta} K^{X_{1}}(S \Phi) \\
& +C \delta^{\prime} \iint_{\mathscr{R}\left(\tau_{0}, \tau\right) \cap\left\{r \leq \frac{1}{2} t^{*}\right\}}\left(t^{*}\right)^{2} K^{X_{0}}(S \Phi)+C\left(\delta^{\prime}+\epsilon\right) \iint_{\mathscr{R}\left(\tau_{0}, \tau\right) \cap\left\{r \leq r_{Y}^{-}\right\}}\left(t^{*}\right)^{2} K^{N}(S \Phi) \\
& +C\left(\delta^{\prime}\right)^{-1}\left(\int_{\tau_{0}}^{\tau}\left(\int_{\Sigma_{t^{*} \cap\left\{r \geq \frac{1}{2} t^{*}\right\}}} r^{2} G^{2}\right)^{1 / 2} d t^{*}\right)^{2}+C\left(\delta^{\prime}\right)^{-1} \sum_{m=0}^{1} \iint_{\mathscr{R}\left(\tau_{0}, \tau\right) \cap\left\{r \leq \frac{9}{10} t^{*}\right\}}\left(t^{*}\right)^{2} r^{1+\delta}\left(\partial_{t^{*}}^{m} G\right)^{2} \\
& +C\left(\delta^{\prime}\right)^{-1} \sup _{t^{*} \in\left[\tau_{0}, \tau\right]} \int_{\Sigma_{t^{*}} \cap\left\{r_{Y}^{-} \leq r \leq \frac{25}{8} M\right\}}\left(t^{*}\right)^{2} G^{2} .
\end{aligned}
$$

It suffices to check that by Propositions 53, 54 and 55, all terms are acceptable.

We have thus shown the following:

Proposition 61. For all $\eta>0$, there exists $\epsilon>0$ small enough such that for Kerr spacetimes satisfying (3), the following estimates hold:

$$
\begin{aligned}
c \int_{\Sigma_{\tau}} J_{\mu}^{Z, w^{Z}}(S \Phi) & n_{\Sigma_{\tau}}^{\mu}+\tau^{2} \int_{\Sigma_{\tau} \cap\{r \leq \gamma \tau\}} J_{\mu}^{N}(S \Phi) n_{\Sigma_{\tau}}^{\mu} \\
& \leq C \tau^{\eta} \sum_{m=0}^{2} \int_{\Sigma_{\tau_{0}}} J_{\mu}^{Z+C N, w^{Z}}\left(\partial_{t^{*}}^{m} S \Phi\right) n_{\Sigma_{\tau_{0}}}^{\mu}+C \tau^{\eta} \sum_{m+k+j \leq 5} \int_{\Sigma_{\tau_{0}}} J_{\mu}^{Z+C N, w^{Z}}\left(\partial_{t^{*}}^{m} \hat{Y} \widetilde{\Omega}^{j} \Phi\right) n_{\Sigma_{\tau_{0}}}^{\mu}
\end{aligned}
$$

Moreover, for $\tau^{\prime} \leq \tau \leq(1.1) \tau^{\prime}$,

$$
\begin{aligned}
& \iint_{\mathscr{R}\left(\tau^{\prime}, \tau\right) \cap\left\{r \leq r_{Y}^{-}\right\}} K^{N}(S \Phi)+\iint_{\mathscr{R}\left(\tau^{\prime}, \tau\right) \cap\left\{r \leq \frac{1}{2} t^{*}\right\}} K^{X_{0}}(S \Phi) \\
& \leq C \tau^{-2+\eta} \sum_{m=0}^{2} \int_{\Sigma_{\tau_{0}}} J_{\mu}^{Z+C N, w^{Z}}\left(\partial_{t^{*}}^{m} S \Phi\right) n_{\Sigma_{\tau_{0}}}^{\mu}+C \tau^{-2+\eta} \sum_{m+k+j \leq 5} \int_{\Sigma_{\tau_{0}}} J_{\mu}^{Z+C N, w^{Z}}\left(\partial_{t^{*}}^{m} \hat{Y} \widetilde{\Omega}^{j} \Phi\right) n_{\Sigma_{\tau_{0}}}^{\mu} .
\end{aligned}
$$

and

$$
\begin{aligned}
& \iint_{\mathscr{R}\left(\tau^{\prime}, \tau\right)} \cap\left\{r \leq \frac{1}{2} t^{*}\right\} \\
& \quad K^{X_{1}}(S \Phi) \\
& \leq C \tau^{-2+\eta} \sum_{m=0}^{3} \int_{\Sigma_{\tau_{0}}} J_{\mu}^{Z+C N, w^{Z}}\left(\partial_{t^{*}}^{m} S \Phi\right) n_{\Sigma_{\tau_{0}}}^{\mu}+C \tau^{-2+\eta} \sum_{m+k+j \leq 6} \int_{\Sigma_{\tau_{0}}} J_{\mu}^{Z+C N, w^{Z}}\left(\partial_{t^{*}}^{m} \hat{Y} \widetilde{\Omega}^{j} \Phi\right) n_{\Sigma_{\tau_{0}}}^{\mu} .
\end{aligned}
$$

Proof. The first statement is proved by the bootstrap above. Since the bootstrap assumptions are true, the conclusion in Proposition 58 is also true; hence the second statement is true. The third statement makes use of the fact that $K^{X_{1}}$ can be estimated in the same way as $K^{X_{0}}$ with an extra derivative. 


\section{Improved decay for the linear homogeneous wave equation}

To use the estimates for $S \Phi$, we need to integrate along integral curves of $S$. We first find the integral curves by solving the ordinary differential equation

$$
\frac{d r_{S}}{d t_{S}^{*}}=\frac{h\left(r_{S}\right)}{t_{S}^{*}}
$$

where $h\left(r_{S}\right)$ is as in the definition of $S$. Hence the integral curves are given by

$$
\rho:=\frac{\exp \left(\int_{\left(r_{S}\right)_{0}}^{r_{S}} \frac{d r_{S}^{\prime}}{h\left(r_{S}^{\prime}\right)}\right)}{t_{S}^{*}}=\text { constant }
$$

where $r_{0}>2 M$ can be chosen arbitrarily. Let $\sigma=t^{*}$, and consider $\left(\sigma, \rho, x^{A}, x^{B}\right)$ as a new system of coordinates. Notice that

$$
\partial_{\sigma}=\frac{h\left(r_{S}\right)}{t^{*}} \partial_{r_{S}}+\partial_{t_{S}^{*}}=\frac{1}{t^{*}} S
$$

Now for each fixed $\rho$, we have

$$
\Phi^{2}(\tau) \leq \Phi^{2}\left(\tau^{\prime}\right)+\left|\int_{\tau^{\prime}}^{\tau} \frac{1}{\sigma} S\left(\Phi^{2}\right) d \sigma\right|
$$

Integrating along a finite region of $\rho$, we get

$$
\int_{\rho_{1}}^{\rho_{2}} \Phi^{2}(\tau) d \rho \leq \int_{\rho_{1}}^{\rho_{2}} \Phi^{2}\left(\tau^{\prime}\right) d \rho+\int_{\rho_{1}}^{\rho_{2}} \int_{\tau^{\prime}}^{\tau}\left|\frac{2}{\sigma} \Phi S \Phi\right| d \sigma d \rho
$$

We would like to change coordinates back to $\left(t_{S}^{*}, r_{S}, x_{S}^{A}, x_{S}^{B}\right)$. Since $h\left(r_{S}\right)$ is everywhere positive, $(\rho, \tau)$ would correspond to a point with a larger value of $r$ than $\left(\rho, \tau^{\prime}\right)$. Therefore,

$\int_{r_{+}}^{r_{2}} \Phi^{2}(\tau) \frac{\exp \left(\int_{\left(r_{S}\right)_{0}}^{r_{S}} \frac{d r_{S}^{\prime}}{h\left(r_{S}^{\prime}\right)}\right)}{\tau h\left(r_{S}\right)} d r$

$$
\leq \int_{r_{+}}^{r_{2}} \Phi^{2}\left(\tau^{\prime}\right) \frac{\exp \left(\int_{\left(r_{S}\right)_{0}}^{r_{S}} \frac{d r_{S}^{\prime}}{h\left(r_{S}^{\prime}\right)}\right)}{\tau^{\prime} h\left(r_{S}\right)} d r+\int_{\tau^{\prime}}^{\tau} \int_{r_{+}}^{r_{2}}\left|\frac{2}{\sigma} \Phi S \Phi\right| \frac{\exp \left(\int_{\left(r_{S}\right)_{0}}^{r_{S}} \frac{d r_{S}^{\prime}}{h\left(r_{S}^{\prime}\right)}\right)}{t_{S}^{*} h\left(r_{S}\right)} d r d t^{*} .
$$

We have to compare $\exp \left(\int_{\left(r_{S}\right)_{0}}^{r_{S}} \frac{d r_{S}^{\prime}}{h\left(r_{S}^{\prime}\right)}\right) / h\left(r_{S}\right)$ with the volume form. Very close to the horizon, we have $h\left(r_{S}\right)=r_{S}-2 M$. Hence

$$
\frac{\exp \left(\int_{\left(r_{S}\right)_{0}}^{r_{S}} \frac{d r_{S}^{\prime}}{h\left(r_{S}^{\prime}\right)}\right)}{h\left(r_{S}\right)}=e^{\int_{\left(r_{S}\right)_{0}}^{r_{S}} \frac{d r_{S}^{\prime}}{h\left(r_{S}^{\prime}\right)}}\left(\frac{1}{r_{S}-2 M}\right) \sim 1 .
$$

The corresponding expression on the compact set $\left[r_{Y}^{-}, R\right]$ is obviously bounded. Hence we have

$$
\int_{\Sigma_{\tau} \cap\left\{r<r_{2}\right\}} \frac{\Phi^{2}(\tau)}{\tau} \leq C\left(\int_{\Sigma_{\tau^{\prime}} \cap\left\{r<r_{2}\right\}} \frac{\Phi^{2}\left(\tau^{\prime}\right)}{\tau^{\prime}}+\iint_{\mathscr{R}\left(\tau^{\prime}, \tau\right) \cap\left\{r<r_{2}\right\}}\left|\frac{2}{\left(t^{*}\right)^{2}} \Phi S \Phi\right|\right) .
$$

This easily implies the following improved decay for the nondegenerate energy: 
Proposition 62. $\int_{\Sigma_{\tau} \cap\{r<R\}} \Phi^{2} \leq C_{R} \tau^{-1}\left(\iint_{\mathscr{R}_{\left((1.1)^{-1} \tau, \tau\right) \cap\{r<R\}}} \Phi^{2}+\iint_{\mathscr{R}\left((1.1)^{-1} \tau, \tau\right) \cap\{r<R\}}(S \Phi)^{2}\right) R$. Proof. By choosing an appropriate $\tilde{\tau} \in\left[(1.1)^{-1} \tau, \tau\right]$, we have

$$
\int_{\Sigma_{\tilde{\tau}} \cap\{r<R\}} \Phi^{2} \leq C \tau^{-1} \iint_{\mathscr{R}\left((1.1)^{-1} \tau, \tau\right) \cap\{r<R\}} \Phi^{2} .
$$

Now, apply (25) with $\tau^{\prime}=\tilde{\tau}$, we have

$$
\begin{aligned}
\int_{\Sigma_{\tau} \cap\{r<R\}} \Phi^{2} & \leq C \tau\left(\int_{\Sigma_{\tilde{\tau}} \cap\{r<R\}} \frac{\Phi^{2}}{\tilde{\tau}}+\iint_{\mathscr{R}(\tilde{\tau}, \tau) \cap\{r<R\}}\left|\frac{2}{\left(t^{*}\right)^{2}} \Phi S \Phi\right|\right) \\
& \leq C \tau^{-1}\left(\iint_{\mathscr{R}\left((1.1)^{-1} \tau, \tau\right) \cap\{r<R\}} \Phi^{2}+\iint_{\mathscr{R}\left((1.1)^{-1} \tau, \tau\right) \cap\{r<R\}}(S \Phi)^{2}\right),
\end{aligned}
$$

using Cauchy-Schwarz for the second term.

We can now conclude with the improved decay for solutions to the homogeneous wave equation.

Proof of Main Theorem. By Corollaries 43, 62 and 61, we have

$$
\begin{aligned}
\int_{\Sigma_{\tau} \cap\{r<R\}} \Phi^{2} & \leq C_{R} \tau^{-1}\left(\iint_{\mathscr{R}\left((1.1)^{-1} \tau, \tau\right) \cap\{r<R\}} \Phi^{2}+\iint_{\mathscr{R}\left((1.1)^{-1} \tau, \tau\right) \cap\{r<R\}}(S \Phi)^{2}\right) \\
& \leq C_{R} \tau^{-1} \iint_{\mathscr{R}\left((1.1)^{-1} \tau, \tau\right) \cap\{r<R\}}\left(K^{X_{0}}(\Phi)+K^{X_{0}}(S \Phi)\right) \\
& \leq C_{R} \tau^{-3+\eta}\left(\sum_{m=0}^{2} \int_{\Sigma_{\tau_{0}}} J_{\mu}^{Z+C N, w^{Z}}\left(\partial_{t^{*}}^{m} S \Phi\right) n_{\Sigma_{\tau_{0}}}^{\mu}+\sum_{m+k+j \leq 5} \int_{\Sigma_{\tau_{0}}} J_{\mu}^{Z+C N, w^{Z}}\left(\partial_{t^{*}}^{m} \hat{Y} \widetilde{\Omega}^{j} \Phi\right) n_{\Sigma_{\tau_{0}}}^{\mu}\right)
\end{aligned}
$$

Similarly we can use Proposition 62 for the derivatives of $\Phi$. By Corollary 43, 62 and 61, we have

$$
\begin{aligned}
\int_{\Sigma_{\tau} \cap\{r<R\}}(D \Phi)^{2} & \\
\leq & C_{R} \tau^{-1}\left(\iint_{\mathscr{R}\left((1.1)^{-1} \tau, \tau\right) \cap\{r<R\}}(D \Phi)^{2}+\iint_{\mathscr{R}\left((1.1)^{-1} \tau, \tau\right) \cap\{r<R\}}(S D \Phi)^{2}\right) \\
\leq & C_{R} \tau^{-1} \iint_{\mathscr{R}\left((1.1)^{-1} \tau, \tau\right) \cap\{r<R\}}\left(K^{X_{1}}(\Phi)+K^{X_{1}}(S \Phi)\right) \\
& \text { (since we have the commutation }[D, S]=D) \\
\leq & C_{R} \tau^{-3+\eta}\left(\sum_{m=0}^{3} \int_{\Sigma_{\tau_{0}}} J_{\mu}^{Z+C N, w^{Z}}\left(\partial_{t^{*}}^{m} S \Phi\right) n_{\Sigma_{\tau_{0}}}^{\mu}+\sum_{m+k+j \leq 6} \int_{\Sigma_{\tau_{0}}} J_{\mu}^{Z+C N, w^{Z}}\left(\partial_{t^{*}}^{m} \hat{Y} \widetilde{\Omega}^{j} \Phi\right) n_{\Sigma_{\tau_{0}}}^{\mu}\right) .
\end{aligned}
$$

By commuting with $\partial_{t^{*}}$, we get

$$
\begin{aligned}
\int_{\Sigma_{\tau} \cap\{r<R\}}\left(D \partial_{t^{*}}^{\ell} \Phi\right)^{2} & \\
& \leq C_{R} \tau^{-3+\eta}\left(\sum_{m=0}^{\ell+3} \int_{\Sigma_{\tau_{0}}} J_{\mu}^{Z+C N, w^{Z}}\left(\partial_{t^{*}}^{m} S \Phi\right) n_{\Sigma_{\tau_{0}}}^{\mu}+\sum_{m+k+j \leq \ell+6} \int_{\Sigma_{\tau_{0}}} J_{\mu}^{Z+C N, w^{Z}}\left(\partial_{t^{*}}^{m} \hat{Y} \widetilde{\Omega}^{j} \Phi\right) n_{\Sigma_{\tau_{0}}}^{\mu}\right) .
\end{aligned}
$$


Without loss of generality, we can take $R>\frac{23}{8} M$. Then, by Proposition 47,

$$
\begin{aligned}
& \sum_{j+m \leq \ell} \int_{\Sigma_{\tau} \cap\left\{r \leq r_{Y}^{+}\right\}} J_{\mu}^{N}\left(\partial_{t^{*}}^{j} \hat{Y}^{m} \Phi\right) n_{\Sigma_{\tau}}^{\mu} \\
& \quad \leq C_{R} \tau^{-3+\eta}\left(\sum_{m=0}^{\ell+3} \int_{\Sigma_{\tau_{0}}} J_{\mu}^{Z+C N, w^{Z}}\left(\partial_{t^{*}}^{m} S \Phi\right) n_{\Sigma_{\tau_{0}}}^{\mu}+\sum_{m+k+j \leq \ell+6} \int_{\Sigma_{\tau_{0}}} J_{\mu}^{Z+C N, w^{Z}}\left(\partial_{t^{*}}^{m} \hat{Y} \widetilde{\Omega}^{j} \Phi\right) n_{\Sigma_{\tau_{0}}}^{\mu}\right) .
\end{aligned}
$$

Hence, by Proposition 44 and 45,

$$
\begin{aligned}
& \sum_{j=0}^{\ell} \int_{\Sigma_{\tau} \cap\{r \leq R\}}\left(D^{j} \Phi\right)^{2} \\
& \quad \leq C_{R} \tau^{-3+\eta}\left(\sum_{m=0}^{\ell+2} \int_{\Sigma_{\tau_{0}}} J_{\mu}^{Z+C N, w^{Z}}\left(\partial_{t^{*}}^{m} S \Phi\right) n_{\Sigma_{\tau_{0}}}^{\mu}+\sum_{m+k+j \leq \ell+5} \int_{\Sigma_{\tau_{0}}} J_{\mu}^{Z+C N, w^{Z}}\left(\partial_{t^{*}}^{m} \hat{Y} \widetilde{\Omega}^{j} \Phi\right) n_{\Sigma_{\tau_{0}}}^{\mu}\right) .
\end{aligned}
$$

The pointwise decay statement follows from standard Sobolev embedding.

\section{Discussion}

Out main paper holds in the set $\left\{r_{+} \leq r \leq R\right\}$ for any fixed $R$. It is however interesting also to derive the same estimates, for example, in the set $\left\{r_{+} \leq r \leq \frac{1}{2} t^{*}\right\}$. This can be achieved by proving the full decay result when we commuted the equation with $\widetilde{\Omega}^{\ell}$. Using this we can prove (with more loss in derivatives) that

$$
|\Phi| \leq C E\left(t^{*}\right)^{-3 / 2+\eta} r^{\eta} \quad \text { and } \quad|D \Phi| \leq C E\left(t^{*}\right)^{-3 / 2+\eta} r^{-\frac{1}{2}+\eta}
$$

for $r \leq \frac{1}{2} t^{*}$. This will be useful in studying nonlinear problems. This decay rate will be proved as a corollary in our forthcoming paper on the null condition.

\section{Acknowledgments}

I thank my advisor Igor Rodnianski for continual support and encouragement and for many enlightening discussions. I thank Gustav Holzegel for very helpful comments on the manuscript. I thank also an anonymous referee for many suggestions to improve the manuscript.

\section{References}

[Alinhac 2009] S. Alinhac, "Energy multipliers for perturbations of the Schwarzschild metric", Comm. Math. Phys. 288:1 (2009), 199-224. MR 2010b:58038 Zbl 1196.53053

[Andersson and Blue 2009] L. Andersson and P. Blue, "Hidden symmetries and decay for the wave equation on the Kerr spacetime”, preprint, 2009. arXiv 0908.2265

[Blue and Soffer 2006] P. Blue and A. Soffer, "Improved decay rates with small regularity loss for the wave equation about a Schwarzschild black hole”, preprint, 2006. arXiv math.AP/0612168

[Blue and Sterbenz 2006] P. Blue and J. Sterbenz, "Uniform decay of local energy and the semi-linear wave equation on Schwarzschild space”, Comm. Math. Phys. 268:2 (2006), 481-504. MR 2007i:58037 Zbl 1123.58018 
[Carter 1968] B. Carter, "Global structure of the Kerr family of gravitational fields", Phys. Rev., II. Ser. 174:5 (1968), 15591571. Zbl 0167.56301

[Christodoulou and Klainerman 1993] D. Christodoulou and S. Klainerman, The global nonlinear stability of the Minkowski space, Princeton Mathematical Series 41, Princeton University Press, Princeton, NJ, 1993. MR 95k:83006 Zbl 0827.53055

[Dafermos and Rodnianski 2008] M. Dafermos and I. Rodnianski, "Lectures on black holes and linear waves", preprint, 2008. arXiv 0811.0354

[Dafermos and Rodnianski 2009] M. Dafermos and I. Rodnianski, "The red-shift effect and radiation decay on black hole spacetimes”, Comm. Pure Appl. Math. 62:7 (2009), 859-919. MR 2011b:83059 Zbl 1169.83008

[Dafermos and Rodnianski 2010] M. Dafermos and I. Rodnianski, "A new physical-space approach to decay for the wave equation with applications to black hole spacetimes", pp. 421-432 in XVIth International Congress on Mathematical Physics (Prague, 2009), edited by P. Exner, World Scientific, Hackensack, NJ, 2010. MR 2012e:58051 Zbl 1211.83019

[Dafermos and Rodnianski 2011] M. Dafermos and I. Rodnianski, "A proof of the uniform boundedness of solutions to the wave equation on slowly rotating Kerr backgrounds", Invent. Math. 185:3 (2011), 467-559. MR 2827094 Zbl 1226.83029

[Donninger et al. 2011] R. Donninger, W. Schlag, and A. Soffer, "A proof of Price's law on Schwarzschild black hole manifolds for all angular momenta”, Adv. Math. 226:1 (2011), 484-540. MR 2012d:58043 Zbl 1205.83041 arXiv 0908.4292

[Finster et al. 2006] F. Finster, N. Kamran, J. Smoller, and S.-T. Yau, "Decay of solutions of the wave equation in the Kerr geometry”, Comm. Math. Phys. 264:2 (2006), 465-503. MR 2007b:83019 Zbl 1194.83015

[Finster et al. 2008] F. Finster, N. Kamran, J. Smoller, and S.-T. Yau, "Erratum to [Finster et al. 2006]", Comm. Math. Phys. 280:2 (2008), 563-573. MR 2009c:83008 Zbl 1194.83014

[Hartle and Wilkins 1974] J. B. Hartle and D. C. Wilkins, "Analytic properties of the Teukolsky equation", Comm. Math. Phys. 38 (1974), 47-63. MR $50 \# 3848$

[Kay and Wald 1987] B. S. Kay and R. M. Wald, "Linear stability of Schwarzschild under perturbations which are nonvanishing on the bifurcation 2-sphere", Classical Quantum Gravity 4:4 (1987), 893-898. MR 88m:83043 Zbl 0647.53065

[Klainerman and Sideris 1996] S. Klainerman and T. C. Sideris, "On almost global existence for nonrelativistic wave equations in 3D”, Comm. Pure Appl. Math. 49:3 (1996), 307-321. MR 96m:35231 Zbl 0867.35064

[Kronthaler 2007] J. Kronthaler, "Decay rates for spherical scalar waves in the Schwarzschild geometry", preprint, 2007. arXiv 0709.3703

[Lindblad and Rodnianski 2005] H. Lindblad and I. Rodnianski, "Global existence for the Einstein vacuum equations in wave coordinates", Comm. Math. Phys. 256:1 (2005), 43-110. MR 2006b:83020 Zbl 1081.83003

[Luk 2010] J. Luk, "Improved decay for solutions to the linear wave equation on a Schwarzschild black hole", Ann. Henri Poincaré 11:5 (2010), 805-880. MR 2736525 Zbl 1208.83068

[Machedon and Stalker 2002] M. Machedon and J. Stalker, "Decay of solutions to the wave equation on a spherically symmetric background", preprint, 2002.

[Morawetz 1975] C. S. Morawetz, Notes on time decay and scattering for some hyperbolic problems, Regional Conference Series in Applied Mathematics 19, Society for Industrial and Applied Mathematics, Philadelphia, 1975. MR 58 \#11968 Zbl 0303.35002

[Press and Teukolsky 1973] W. H. Press and S. A. Teukolsky, "Pertubations of a rotating black hole, II: Dynamical stability of the Kerr metric", Astrophys. J. 185 (1973), 649-673.

[Sterbenz 2005] J. Sterbenz, "Angular regularity and Strichartz estimates for the wave equation", Int. Math. Res. Not. 2005:4 (2005), 187-231. MR 2006i:35212 Zbl 1072.35048

[Tataru 2009] D. Tataru, "Local decay of waves on asymptotically flat stationary space-times", preprint, 2009. arXiv 0910.5290

[Tataru and Tohaneanu 2011] D. Tataru and M. Tohaneanu, "A local energy estimate on Kerr black hole backgrounds", Int. Math. Res. Not. 2011:2 (2011), 248-292. MR 2012a:58050 Zbl 1209.83028 arXiv 0810.5766

[Tohaneanu 2012] M. Tohaneanu, "Strichartz estimates on Kerr black hole backgrounds", Trans. Amer. Math. Soc. 364:2 (2012), 689-702. MR 2846348 Zbl 1234.35275 arXiv 0910.1545

[Wald 1979] R. M. Wald, "Note on the stability of the Schwarzschild metric", J. Math. Phys. 20:6 (1979), 1056-1058. MR 80c:83012a 
[Whiting 1989] B. F. Whiting, "Mode stability of the Kerr black hole”, J. Math. Phys. 30:6 (1989), 1301-1305. MR 90m:83038 Zbl 0689.53041

Received 5 Oct 2010. Revised 25 Mar 2011. Accepted 21 Apr 2011.

JONATHAN LUK: jluk@math.princeton.edu

Department of Mathematics, Princeteon University, Fine Hall, Washington Road, Princeton, NJ 08544, United States 


\title{
Analysis \& PDE
}

\author{
msp.berkeley.edu/apde
}

EDITORS

EDITOR-IN-CHIEF

Maciej Zworski

University of California

Berkeley, USA

BOARD OF EDITORS

\begin{tabular}{|c|c|c|c|}
\hline Michael Aizenman & $\begin{array}{l}\text { Princeton University, USA } \\
\text { aizenman@math.princeton.edu }\end{array}$ & Nicolas Burq & $\begin{array}{l}\text { Université Paris-Sud 11, France } \\
\text { nicolas.burq@math.u-psud.fr }\end{array}$ \\
\hline Luis A. Caffarelli & $\begin{array}{l}\text { University of Texas, USA } \\
\text { caffarel@math.utexas.edu }\end{array}$ & un-Yung Alice Chang & $\begin{array}{l}\text { Princeton University, USA } \\
\text { chang@math.princeton.edu }\end{array}$ \\
\hline Michael Christ & $\begin{array}{l}\text { University of California, Berkeley, USA } \\
\text { mchrist@math.berkeley.edu }\end{array}$ & Charles Fefferman & $\begin{array}{l}\text { Princeton University, USA } \\
\text { cf@math.princeton.edu }\end{array}$ \\
\hline Ursula Hamenstaedt & $\begin{array}{l}\text { Universität Bonn, Germany } \\
\text { ursula@math.uni-bonn.de }\end{array}$ & Nigel Higson & $\begin{array}{l}\text { Pennsylvania State Univesity, USA } \\
\text { higson@ math.psu.edu }\end{array}$ \\
\hline Vaughan Jones & $\begin{array}{l}\text { University of California, Berkeley, USA } \\
\text { vfr@math.berkeley.edu }\end{array}$ & Herbert Koch & $\begin{array}{l}\text { Universität Bonn, Germany } \\
\text { koch@math.uni-bonn.de }\end{array}$ \\
\hline Izabella Laba & $\begin{array}{l}\text { University of British Columbia, Canada } \\
\text { ilaba@math.ubc.ca }\end{array}$ & Gilles Lebeau & $\begin{array}{l}\text { Université de Nice Sophia Antipolis, France } \\
\text { lebeau@unice.fr }\end{array}$ \\
\hline László Lempert & $\begin{array}{l}\text { Purdue University, USA } \\
\text { lempert@math.purdue.edu }\end{array}$ & Richard B. Melrose & $\begin{array}{l}\text { Massachussets Institute of Technology, USA } \\
\text { rbm@math.mit.edu }\end{array}$ \\
\hline Frank Merle & $\begin{array}{l}\text { Université de Cergy-Pontoise, France } \\
\text { Frank.Merle@u-cergy.fr }\end{array}$ & William Minicozzi II & $\begin{array}{l}\text { Johns Hopkins University, USA } \\
\text { minicozz@ math.jhu.edu }\end{array}$ \\
\hline Werner Müller & $\begin{array}{l}\text { Universität Bonn, Germany } \\
\text { mueller@math.uni-bonn.de }\end{array}$ & Yuval Peres & $\begin{array}{l}\text { University of California, Berkeley, USA } \\
\text { peres@stat.berkeley.edu }\end{array}$ \\
\hline Gilles Pisier & $\begin{array}{l}\text { Texas A\&M University, and Paris } 6 \\
\text { pisier@math.tamu.edu }\end{array}$ & Tristan Rivière & $\begin{array}{l}\text { ETH, Switzerland } \\
\text { riviere@math.ethz.ch }\end{array}$ \\
\hline Igor Rodnianski & $\begin{array}{l}\text { Princeton University, USA } \\
\text { irod@math.princeton.edu }\end{array}$ & Wilhelm Schlag & $\begin{array}{l}\text { University of Chicago, USA } \\
\text { schlag@math.uchicago.edu }\end{array}$ \\
\hline Sylvia Serfaty & $\begin{array}{l}\text { New York University, USA } \\
\text { serfaty@ cims.nyu.edu }\end{array}$ & Yum-Tong Siu & $\begin{array}{l}\text { Harvard University, USA } \\
\text { siu@math.harvard.edu }\end{array}$ \\
\hline Terence Tao & $\begin{array}{l}\text { University of California, Los Angeles, USA } \\
\text { tao@math.ucla.edu }\end{array}$ & A Michael E. Taylor & $\begin{array}{l}\text { Univ. of North Carolina, Chapel Hill, USA } \\
\text { met@math.unc.edu }\end{array}$ \\
\hline Gunther Uhlmann & $\begin{array}{l}\text { University of Washington, USA } \\
\text { gunther@math.washington.edu }\end{array}$ & András Vasy & $\begin{array}{l}\text { Stanford University, USA } \\
\text { andras@math.stanford.edu }\end{array}$ \\
\hline Virgil Voiculescu & $\begin{array}{l}\text { University of California, Berkeley, USA } \\
\text { dvv@math.berkeley.edu }\end{array}$ & Steven Zelditch & $\begin{array}{l}\text { Northwestern University, USA } \\
\text { zelditch@math.northwestern.edu }\end{array}$ \\
\hline
\end{tabular}

\section{PRODUCTION}

contact@msp.org

Silvio Levy, Scientific Editor

Sheila Newbery, Senior Production Editor

See inside back cover or msp.berkeley.edu/apde for submission instructions.

The subscription price for 2012 is US \$140/year for the electronic version, and \$240/year for print and electronic. Subscriptions, requests for back issues from the last three years and changes of subscribers address should be sent to Mathematical Sciences Publishers, Department of Mathematics, University of California, Berkeley, CA 94720-3840, USA.

Analysis \& PDE, at Mathematical Sciences Publishers, Department of Mathematics, University of California, Berkeley, CA 94720-3840 is published continuously online. Periodical rate postage paid at Berkeley, CA 94704, and additional mailing offices.

APDE peer review and production are managed by EditFLOW ${ }^{\mathrm{TM}}$ from Mathematical Sciences Publishers.

PUBLISHED BY

mathematical sciences publishers

http://msp.org/

A NON-PROFIT CORPORATION

Typeset in IATEX

Copyright $(2012$ by Mathematical Sciences Publishers 


\section{ANALYSIS \& PDE}

\section{Volume $5 \quad$ No. $3 \quad 2012$}

On some microlocal properties of the range of a pseudodifferential operator of principal type 423 JENS WITTSTEN

Blow-up solutions on a sphere for the 3D quintic NLS in the energy space JUSTIN HOLMER and SVETLANA ROUDENKO

Sharp geometric upper bounds on resonances for surfaces with hyperbolic ends DAVID BORTHWICK

A vector field method approach to improved decay for solutions to the wave equation on a slowly rotating Kerr black hole JONATHAN LUK

On the Bogolyubov-Ruzsa lemma TOM SANDERS

Real analyticity away from the nucleus of pseudorelativistic Hartree-Fock orbitals

Anna Dall'Acqua, Søren Fournais, Thomas ØstergaArd Sørensen and EDGARDO STOCKMEYER

Semiclassical trace formulas and heat expansions

YVES COLIN DE VERDIÈRE 